\title{
Untersuchung zur Bedeutung von Bewegungsangst bei chronischen Rückenschmerzen
}

\author{
Dissertation \\ zur Erlangung des Doktorgrades \\ der Mathematisch-Naturwissenschaftlichen Fakultäten \\ der Georg-August-Universität zu Göttingen
}

vorgelegt von
Uta Kronshage
aus Göttingen

Göttingen 2001 
D7

Referentin: Prof. Dr. B. Kröner-Herwig

Korreferent: Prof. Dr. T. Rammsayer

Tag der mündlichen Prüfung: 31.10.2001 


\section{Widmung}

Ich widme diese Arbeit meinen Großmüttern, deren Intelligenz und Klugheit nie durch Bildungsabschlüsse anerkannt wurde. Und meiner Mutter, die auf den Doktortitel verzichtet hat, um uns Kinder aufzuziehen. 


\section{Theoretischer Hintergrund}

2.1 Chronischer Rückenschmerz

$\begin{array}{lll}2.2 & \text { Chronifizierungsfaktoren } & \mathbf{7}\end{array}$

2.2.1 Soziodemographische Faktoren und Bedingungen des Arbeitsplatzes $\quad 7$

Operante Faktoren, Angst vor Schmerz und Vermeidung

Bewegungsangst und Fear-Avoidance Beliefs

2.3 Untersuchung der Konzepte Bewegungsangst und Fear-Avoidance Beliefs

2.3.2 Untersuchung der Konzepte Bewegungsangst und Fear-Avoidance Beliefs unter Verwendung von Fragebögen

\section{Methodik}

3.1 Versuchsplan und unabhängige Variablen

Abhängige Variablen 39 39 Covariation Bias

$\begin{array}{lll}3.3 & \text { Probanden } & 46\end{array}$

$\begin{array}{ll}\text { Räumliche Bedingungen } & 47\end{array}$

\section{Ergebnisse}

4.1 $\quad$ Eingangsmerkmale der Stichprobe

4.3 Überprüfung der State-Angst, der Arousal-Einschätzung und des Einflusses der Reihenfolge (Blocks) auf die Ergebnisse der Hypothesenprüfung 
5.1 Untersuchung der Konzepte Bewegungsangst und Fear-Avoidance Beliefs unter Verwendung des Startle-Paradigmas

5.2 Untersuchung der Konzepte Bewegungsangst und Fear-Avoidance Beliefs unter Verwendung des Covariation Bias

5.3 Untersuchung der Konzepte Bewegungsangst und Fear-Avoidance Beliefs unter Verwendung von Fragebögen

$5.4 \quad$ Gesamtdiskussion

6. Literatur

7. ANHANG 


\section{Zusammenfassung}

In vielen Untersuchungen hat sich gezeigt, dass Patienten mit chronischen Rückenschmerzen in hohem Maße dazu neigen, bestimmte Bewegungen zu vermeiden. Diese Vermeidung kann zu generellem Rückzugs- und Schonverhalten führen, was wiederum einen schlechten körperlichen Zustand bzw. Depressivität aufgrund eines Mangels an sozialen Verstärkern zur Folge haben kann. Bisher ist jedoch noch nicht gut untersucht, ob die Patienten die Bewegungen vermeiden, weil sie tatsächlich Angst vor diesen Bewegungen erleben oder ob sie eher aufgrund von kognitiven Schemata über die Schädlichkeit von Bewegungen handeln. Um dieser Frage nachzugehen werden im experimentellen Teil der vorliegenden Studie zwei Paradigmen verwendet, die sich bei der Untersuchung von Phobien bereits mehrfach bewährt haben: das Startle-Paradigma und der Covariation Bias. Dabei reflektiert die Startle-Reaktion (die Ableitung des Schreckreflexes am Augenmuskel) die Aktivierung des neuronalen Vermeidungs/Fluchtsystems und gibt Aufschluss über die emotionale Situation, in der sich eine Person befindet. Der Covariation Bias (die Angabe über die Assoziation eines Stimulus mit einem aversiven Reiz) gibt Hinweise auf die Informationsverarbeitung einer Person und erlaubt Rückschlüsse darüber, inwieweit bestimmte Reize alsbesonders bedrohlich bewertet werden.

Untersucht werden 36 Patienten mit chronischen Rückenschmerzen, sowie 18 Patienten mit chronischen Kopfschmerzen und 18 gesunde Probanden. Alle Versuchspersonen werden mit Bildern von rückenschmerzrelevanten und von angenehmen Bewegungen konfrontiert und ihre Startle-Reaktion bzw. der Covariation Bias erhoben. Es wird davon ausgegangen, dass die Patienten mit chronischen Rückenschmerzen bei der Betrachtung der rückenschmerzrelevanten Bilder gegenüber der Kontrollgruppe eine erhöhte StartleReaktion zeigen und einen erhöhten Covariation Bias, d.h. dass sie häufiger fälschlich davon ausgehen, dass diese Bilder mit einem aversiven Reiz (lauter Ton) gemeinsam präsentiert worden sind. Zudem wird erwartet dass sie diese Bilder negativer bewerten als die Personen der beiden Kontrollgruppen. Da im Zusammenhang mit Angst vor Bewegungen auch das Konzept der Fear-Avoidance Beliefs diskutiert wird, wird zudem untersucht, ob sich die Probanden in ihren Reaktionen unterscheiden, je nachdem, ob sie ausgeprägte Fear-Avoidance Beliefs zeigen oder nicht. 
Um Aufschluss darüber zu erhalten, ob mit dem Fear-Avoidance Beliefs Questionnaire eher unmittelbar erlebte Angst vor Schmerzen erfasst wird oder eher allgemeine Einstellungen zur Notwendigkeit von Rückzug und Vermeidung bei Schmerzen, wird im zweiten Teil der Untersuchung der Zusammenhang des Fear-Avoidance Beliefs Questionnaires mit zwei selbst erstellten Fragebögen erhoben, die diese psychologischen Prozesse abbilden sollen.

Die untersuchten Rückenschmerzpatienten zeigen keine Erhöhung der Startle-Reaktion bei der Konfrontation mit rückenschmerzrelevanten Bildern. Das deutet darauf hin, das die Patienten in diesem Zusammenhang keine akute Angst erleben. Die Patienten schätzen die Bilder jedoch negativer ein als die Personen der beiden Kontrollgruppen und sie zeigen einen erhöhten Covariation Bias. Offenbar bewerten also die Patienten mit Rückenschmerzen die rückenschmerzrelevanten Bewegungen als bedrohlicher bzw. schädlicher als die Probanden der beiden Kontrollgruppen. Ob die Probanden ausgeprägte Fear-Avoidance Beliefs aufweisen oder nicht, hat dabei keinen Einfluss auf die Reaktion der Rückenschmerzpatienten. Die Analyse der Fragebogenzusammenhänge ergibt kein klares Bild darüber, welche Prozesse der Fear-Avoidance Beliefs Questionnaire erfasst. Offenbar scheint jedoch das Konzept von Gefährlichkeit bestimmter Bewegungen, das die Rückenschmerzpatienten hier zeigen, von FearAvoidance Beliefs relativ unabhängig zu sein. Die Ergebnisse deuten darauf hin, dass das Vermeidungsverhalten der Patienten mit chronischen Rückenschmerzen weniger durch unmittelbar erlebte Angst vor Bewegungen motiviert wird, sondern eher durch kognitive Schemata über die Gefährlichkeit bestimmter Bewegungen. 


\section{Theoretischer Hintergrund}

\subsection{Chronischer Rückenschmerz}

Rückenschmerz ist ein weit verbreitetes Phänomen, das es zu allen Zeiten gegeben hat (Allan \& Waddell, 1989). Neuere Studien in der Bundesrepublik ergaben, dass auf die Frage „Leiden Sie heute an Rückenschmerzen?“ etwa $40 \%$ der Befragten mit „Ja“ antworten. Rückenschmerzen "im letzten Jahr" gehabt zu haben, geben etwa $70 \%$ der Befragten an und überhaupt schon einmal unter Rückenschmerzen gelitten zu haben, ungefähr 80 \% (Raspe \& Kohlmann, 1998). Diese Zahlen enthalten allerdings keinerlei Informationen darüber, welche Auswirkungen die angegebenen Rückenschmerzen haben. So geht aus diesen Zahlen nicht hervor, wie stark beeinträchtigt die Person durch die Rückenschmerzen ist, ob sie häufiger wegen dieser Schmerzen krank geschrieben wird oder gar generell erwerbsunfähig aufgrund der Schmerzen ist.

In der Bundesrepublik hat es in den letzten Jahren einen deutlichen Anstieg bei der Anzahl der Arbeitsfehltage und der Frühberentungen aufgrund von Rückenschmerzen gegeben (Raspe \& Kohlmann, 1998). Das bedeutet natürlich auch einen Anstieg der Kosten im Gesundheitswesen. Allein die Kosten der Rentenversicherungsträger für Rehabilitationsmaßnahmen im Zusammenhang mit Rückenschmerzen werden auf über 2 Milliarden DM im Jahr beziffert (Jäckel \& Gerdes, 1998). Der gleiche Trend einer massiven Erhöhung der Ausgaben, die durch Krankschreibungen und Frühberentungen bei Rückenschmerzen entstehen, findet sich in den meisten Industrieländern. Besonders gut dokumentiert ist dies für die USA, Großbritannien und Schweden (Fordyce 1995, Waddell 1998). Ob diese Entwicklung jedoch auf einen Anstieg in den Prävalenzraten von Rückenschmerzen zurückzuführen ist, ist nicht eindeutig nachweisbar, da es keine epidemiologischen Längsschnittstudien zu diesem Aspekt gibt. Raspe und Kohlmann (1998) kommen aufgrund ihrer Sicht verschiedener Studien zu der Ansicht, dass ein leichter Anstieg in den Prävalenzraten wahrscheinlich ist, während Waddell (1998) betont, dass weder die Prävalenzraten angestiegen sind noch die Pathologie sich verändert hat. Es lässt sich also eine deutliche Zunahme der sozialmedizinischen Folgen von Rückenschmerzen feststellen, ohne dass ein linearer Zusammenhang mit der Zunahme der Häufigkeit bzw. der Stärke von Rückenschmerzen zu finden ist. So stellt sich die Frage, welche anderen Aspekte zu dem beschriebenen Phänomen beitragen. In den letzten Jahren sind vielerlei Forschungsanstrengungen unternommen worden, um 
dieser Frage nachzugehen. Um ein genaueres Verständnis des Phänomens „Rückenschmerzen“ zu bekommen, sind dabei verschiedene Differenzierungen vorgenommen worden, die zunächst vorgestellt werden sollen.

Eine der wichtigsten Unterscheidungen ist die zwischen dem sogenannten spezifischen und dem unspezifischen Rückenschmerz (Waddell 1996). Der spezifische Rückenschmerz läßt sich eindeutig zurückführen auf einen pathologischen Organbefund, sei es ein Bandscheibenprolaps, eine Reizung der Nervenwurzel oder eine Erkrankung wie Morbus Bechterew. Oft, wenn auch nicht immer, lässt sich aus der Diagnose eine spezifische Behandlung herleiten.

Sehr viel komplizierter liegt der Fall beim unspezifischen Rückenschmerz, bei dem nach Raspe und Kohlmann (1998) mindestens drei Punkte geklärt sein müssen:

Es ist keine nosologische Diagnose zu stellen, der Pathomechanismus der Nozizeption ist nicht zu klären und die irritierte Struktur ist nicht identifizierbar.

Entgegen der Überzeugung der meisten Laien und auch vieler Fachleute ist dieser unspezifische Rückenschmerz sehr viel häufiger als der spezifische. Nach Angaben einer Arbeitsgruppe der International Association for the Study of Pain (IASP) sind etwa 90\% der Rückenschmerzen als unspezifisch zu betrachten. Die Behandlung dieser unspezifischen Rückenschmerzen ist aufgrund der unbekannten Ätiologie oft außerordentlich unbefriedigend und langwierig (Fordyce 1995). In der Hoffnung, doch noch irgendwann eine eindeutige und behandelbare Ursache für ihre Schmerzen zu finden, wechseln die Patienten häufig den Arzt oder versuchen immer neue alternative Behandlungsansätze. Die Ärzte fühlen sich bei der Behandlung der unspezifischen Rückenschmerzen oft hilflos, da es keine klaren Therapierichtlinien gibt. Die Überforderung durch die Situation, therapeutisch wirksam sein zu wollen, ohne zu wissen wie, äußert sich häufig in uneindeutigen Diagnosen, nicht indizierten Operationen und anderen wenig sinnvollen Behandlungsversuchen.

Der Rückenschmerz ist aber nicht nur als spezifisch versus unspezifisch zu klassifizieren, eine andere wichtige Unterscheidung ist die zwischen akutem und chronischem Schmerz. Akuter Rückenschmerz ist kurzfristig, und auch wenn er zeitweilig außerordentlich beeinträchtigend sein kann, hat er zumeist keine längerfristigen Auswirkungen auf die Person. Ungefähr $90 \%$ der akuten Rückenprobleme klingen nach kurzer Zeit vollständig wieder ab (Fordyce 1995). 
Der chronische Schmerz wird zunächst charakterisiert durch die Zeitdauer. Hier werden unterschiedliche Kriterien angegeben, zumeist zwischen 3 und 6 Monaten, die der Schmerz bereits andauern muss, um als chronisch bezeichnet zu werden (Kröner-Herwig 1999). Der chronische Schmerz ist häufig unspezifisch, und wenn er spezifisch ist, ist die Schmerzursache oft nicht ohne weiteres kurativ zu behandeln (wie z.B. beim Morbus Bechterew). Der chronische Schmerz begleitet also den Menschen über lange Zeit und kann massive Auswirkungen auf die Person haben. Es kommt oft zu regelrechten Patientenkarrieren, bei denen die Betroffenen jahrelang einen Großteil ihrer Lebensenergie darauf verwenden, endlich eine hilfreiche Behandlungsmethode für ihre Rückenschmerzen zu finden. Das erklärt unter anderem, warum die Gruppe der chronifizierten Rückenschmerzpatienten, die ungefähr 5-25\% aller Rückenschmerzpatienten ausmacht, $75-95 \%$ der durch Rückenschmerz im Gesundheitswesen anfallenden Gesamtkosten verursacht (Kosten für Behandlungen und insbesondere Kosten für Arbeitsausfälle, van Doorn, 1998). Ein weiterer Faktor, der nachvollziehbar macht, warum insbesondere der chronische Rückenschmerz zu so hohen sozialmedizinischen Kosten führt, ist die Tatsache, dass nach 6 Monaten Arbeitsunfähigkeit die Wahrscheinlichkeit, wieder an den Arbeitsplatz zurückzukehren, drastisch abnimmt, nach 2 Jahren gibt es kaum noch Patienten, die ihre Arbeit wieder aufnehmen (Waddell 1998).

Aufgrund dieser Fakten wird dem Phänomen des chronischen unspezifischen Rückenschmerzes in letzter Zeit besondere Aufmerksamkeit gewidmet. Dabei wird deutlich, dass zur Betrachtung von Faktoren, die zur Chronifizierung bzw. zur Aufrechterhaltung von Schmerzen beitragen, das alte medizinische Kausalmodell wenig hilfreich ist und kaum nützliche Erklärungen und Ansätze liefert. Statt dessen wird vorgeschlagen, ein bio-psycho-soziales Modell von Schmerzen heranzuziehen (Waddell 1996, 1998). Bei diesem Modell wird die Störung, in diesem Fall der Schmerz, von verschiedenen Perspektiven aus betrachtet. Die biologische Perspektive konzentriert sich auf den organischen Aspekt der Störung, auf der psychologische Seite werden u.a. Faktoren wie Copingstrategien, psychologische Stressfaktoren oder Schmerzverhalten beleuchtet, während man sich bei der Betrachtung der sozialen Dimension z.B. mit Aspekten der sozialen Umwelt, der Arbeitssituation und dem Gesundheitssystem beschäftigt. Dieser Betrachtungsweise ist auch die Internationale Gesundheitsorganisation (WHO) bei ihrer International Classification of Impairment, Disability and Handicap (ICIDH, 1980) gefolgt . Diese Klassifikation beschreibt die 
verschiedenen Krankheitsfolgen, die im Zusammenhang mit Störungen, in diesem Fall dem Rückenschmerz, auftreten können. Der Begriff der Schädigung (impairment) drückt aus, dass die körperliche oder seelische Unversehrtheit beeinträchtigt ist. Funktionsbeeinträchtigung (disability) bedeutet eine Einschränkung der Möglichkeiten, bestimmte Verhaltensweisen auszuführen und Behinderung (handicap) bezieht sich auf die Einschränkung in der Ausübung einer der Lebenssituation angepassten sozialen Rolle (Harper, Harper, Lambert, Andrews, Lo et al. 1992). Mittlerweile wurde diese Klassifikation noch ausgeweitet, so dass mit der ICIDH-2 von 1998 ein umfangreiches Klassifikationssystem zur Verfügung steht, mit dem der gesundheitliche Status einer Person im Sinne des bio-psycho-sozialen Modells sehr genau beschrieben werden kann. Während der Begriff der Chronifizierung anfangs fast ausschließlich an der Zeitdauer einer Störung festgemacht wurde, wird mittlerweile eher in den Blick genommen, wie beeinträchtigt sich eine Person durch den Schmerz fühlt und wie stark sie in der Ausübung ihrer sozialen Rolle gehindert ist (Waddell, Newton, Henderson, Sommervile \& Main 1993, Waddell 1998). Wie ein Patient seine Möglichkeiten wahrnimmt, seiner Arbeit, Aktivitäten im Haushalt oder in der Freizeit nachzugehen, wird seinen Umgang mit dem Schmerz stark beeinflussen. Waddell (1998) konnte z.B. zeigen, dass das Ausmaß der Arbeitsfehltage eng mit der erlebten Beeinträchtigung zusammenhängt. Daher erscheint es notwendig, genauer zu betrachten, von welchen Faktoren dieses Ausmaß an Beeinträchtigung abhängt. Zunächst wurde von einem linearen Zusammenhang zwischen Organbefund, Schmerzstärke und Beeinträchtigung ausgegangen. Verschiedenste Studien zeigen jedoch nur geringe Korrelationen zwischen der Schmerzstärke und dem Organbefund (Slater, Hall, Atkinson und Garfin, 1991,Waddell, Sommerville, Henderson \& Newton 1992, Waddell et al. 1993,). So gibt es sowohl Menschen mit massiven Organbefunden ohne Schmerzen als auch Menschen mit massiven Schmerzen ohne Organbefund. Aber auch die Schmerzstärke allein kann das Ausmaß der Beeinträchtigung nur in geringem Maße vorhersagen (Waddell et al. 1993, Kröner-Herwig, Jäkle, Frettlöh, Peters, Seemann, et al. 1996). Manche Menschen mit starken Schmerzen fühlen sich vergleichsweise wenig beeinträchtigt, gehen ihrer Arbeit nach und sind in ihrem alltäglichen Leben wenig eingeschränkt, während andere mit geringeren Schmerzstärken über schwere Beeinträchtigungen berichten. Je beeinträchtigter sich ein Mensch durch die Schmerzen fühlt, desto eher wird er wiederholt zum Arzt gehen, sich krankschreiben lassen oder eine Berentung anstreben. Dies könnte eine Erklärung für die starke Erhöhung der Gesundheitskosten bei relativ 
gleich bleibenden Prävalenzraten sein: möglicherweise hat nicht der Schmerz an sich zugenommen, sondern die durch den Schmerz erlebte Beeinträchtigung. Darüber hinaus spielen eventuell auch noch Faktoren des Gesundheitssystems und des Arbeitsmarktes eine Rolle wie z.B. verstärkte Neigung zu Krankschreibungen oder Frühberentungen aufgrund von Umstrukturierungen auf dem Arbeitsmarkt (Frühberentung statt Arbeitslosigkeit). Zudem scheint es eine gewisse gesamtgesellschaftliche Akzeptanz zu geben, Rückenschmerzen als ernstzunehmende Krankheit anzusehen, die lange Bettruhe nötig macht bzw. zu früher Arbeitsunfähigkeit führt (Waddell 1998). Auf diese Aspekte soll hier jedoch nicht näher eingegangen werden, obwohl sie grundsätzlich nicht außer Acht gelassen werden sollten.

Wenn im Zusammenhang mit chronischen Rückenschmerzen nicht der Schmerz an sich eine Rolle spielt, sondern die Frage, wie beeinträchtigt sich ein Mensch durch seine Schmerzen fühlt, so muss auch die Frage nach Risikofaktoren in diesem Sinne gestellt werden. Um die Frage zu klären, welche Faktoren dazu beitragen, dass ein Schmerz chronifiziert, muss untersucht werden, welche Faktoren dazu beitragen, dass sich der Patient durch seine Schmerzen stark beeinträchtigt fühlt. Bezug nehmend auf das biopsycho-soziale Modell bedeutet das, dass insbesondere die psychologische Seite des Problems untersucht werden muss. Die bisherigen Untersuchungen von Risikofaktoren für die Chronifizierung von Rückenschmerzen haben die Bedeutung der psychologischen Faktoren immer wieder bestätigt. Dass biologische Faktoren der Pathogenese eine eher geringe Rolle spielen, wurde bereits dargestellt. Faktoren, die eher der sozialen Seite des Modells zuzurechnen sind, sollen im folgenden kurz diskutiert werden, bevor psychologische Faktoren ausführlicher dargestellt werden.

\subsection{Chronifizierungsfaktoren}

\subsubsection{Soziodemographische Faktoren und Bedingungen des Arbeitsplatzes}

Die Ergebnisse von Studien über das Geschlecht und Prävalenzraten von Rückenschmerzen zeigen eher keine großen Unterschiede zwischen Männern und Frauen, sowohl bei der Einmonats- als auch bei der Lebenszeitprävalenz (Raspe und Kohlmann 1998, Papageorgiou, Croft, Ferry, Jayson \& Silman 1995). Gewisse Diskrepanzen, die in manchen Untersuchungen auftreten, sind vermutlich eher auf 
verschiedene soziale Faktoren zurückzuführen als auf das Geschlecht, wie z.B. auf unterschiedliche Arbeitsfelder oder auf Unterschiede im sozialen Status (Waddell 1998). In Bezug auf das Alter gibt es verschiedene Angaben, übereinstimmend wird jedoch angenommen, dass es eine besonders hohe Prävalenz von Rückenschmerzen im Alter zwischen 40 und 60 Jahren gibt. Auch wenn sich die Prävalenzraten zwischen 20 und 60 nicht stark unterscheiden, fühlen sich ältere Menschen eher durch den Schmerz beeinträchtigt und geben Rückenschmerz häufiger als Ursache für Krankschreibungen an (Papageorgiou et al. 1995).

Über den Zusammenhang zwischen chronischen Rückenschmerzen und der sozialen Schichtzugehörigkeit gibt es verschiedene, sich teilweise widersprechende Befunde. Relativ eindeutig scheint zu sein, dass der Verlust des Arbeitsplatzes aufgrund von Rückenschmerzen in niedrigeren sozialen Schichten häufiger ist (Walsh, Cruddas und Coggon, 1992). Hier lässt sich jedoch nur schwer feststellen, welche Faktoren sozialer Schichtzugehörigkeit dazu beitragen. Unterschiede in der Art der Arbeit (z.B. monotone Arbeit mit rigiden Körperhaltungen), geringere Arbeitszufriedenheit oder auch generell größere Belastungen durch soziale Benachteiligung könnten hier eine Rolle spielen.

Auch wenn sich bei den soziodemographischen Faktoren einige Zusammenhänge zu chronischen Rückenschmerzen aufzeigen lassen, betont Hasenbring (1999), dass diese als Prädiktoren für langanhaltenden Rückenschmerz generell wenig Varianz aufklären.

Über lange Zeit wurden immer wieder bestimmte Arbeitsbedingungen für die Chronifizierung von Rückenschmerzen verantwortlich gemacht, darunter insbesondere schwere körperliche Arbeit, schweres Heben und häufiges Bücken. Waddell (1998) kommt in seinem Überblick über verschiedene Studien zu diesem Thema zu folgenden Schlussfolgerungen: Generell gibt es keine klaren Hinweise, dass schwere körperliche Arbeit zu chronischem Rückenschmerz führt. Schweres Heben ist möglicherweise in Verbindung mit einer Drehung des Körpers tatsächlich ein Faktor, der zu verstärkten Rückenproblemen führen kann. Ähnliches gilt für langes Autofahren, bei dem langes Sitzen in Verbindung mit Vibrationen den Körper schädigen kann. Menschen mit schwerer körperlicher Arbeit werden häufiger wegen Rückenschmerzen krank geschrieben und kehren nicht so schnell an ihren Arbeitsplatz zurück. Diese Tatsache muss jedoch nicht bedeuten, dass diese Menschen insgesamt stärkere Schmerzen haben. Körperliche Arbeit wird von Rückenschmerzen möglicherweise stärker beeinträchtigt als geistige Arbeit und kann mit Rückenschmerzen weniger gut ausgeübt werden. Menschen 
mit Rückenschmerzen, die einer körperlichen Arbeit nachgehen, kehren deshalb möglicherweise später an den Arbeitsplatz zurück.

In einer groß angelegten Langzeitstudie bei der Firma Boing kommen Bigos, Battie, Spengler, Fisher, Fordyce et al. (1991) zu dem Schluss, dass sowohl soziodemographische Faktoren als auch Faktoren des Arbeitsplatzes alle einen sehr geringen Vorhersagewert für das Auftreten von Rückenschmerzen haben. In der Studie ergaben sich Hinweise, dass das subjektive Erleben der Arbeitssituation und die Arbeitszufriedenheit eine größere Rolle spielen als objektive Faktoren. Die Bedeutung der Arbeitszufriedenheit für den Verlauf von Rückenschmerzerkrankungen betonen auch Linton und Warg (1993), van Poppel, Koes, Deville, Smid und Bouter (1998) und van der Gienzen, Bouter und Nijhuis (2000). Alle drei Arbeitsgruppen konnten zeigen, dass geringe Arbeitszufriedenheit einen stärkeren Prädiktor für die Entwicklung von Rückenschmerzen mit nachfolgender Krankschreibung darstellt als objektive Elemente des Arbeitsplatzes oder soziodemographische Aspekte. Papageorgiou, Macfarlane, Thomas, Croft, Jayson et al. (1997) fanden in ihrer Längsschnittstudie ebenfalls einen Zusammenhang zwischen niedriger Arbeitszufriedenheit und dem Auftreten von Rückenschmerzen, allerdings keinen Zusammenhang zu Krankschreibungen aufgrund dieser Schmerzen.

Mit der Betrachtung der Arbeitszufriedenheit ist bereits eine Perspektive erwähnt, die das subjektive Erleben der Person in den Vordergrund stellt. Diese Sichtweise weist also schon hin auf die psychologische Seite des bio-psycho-sozialen Modells, wobei hier die Übergänge zur sozialen Seite des Modells fließend sind.

Weitere psychologische Faktoren, die im Zusammenhang mit der Chronifizierung von Rückenschmerzen diskutiert werden, sollen nun dargestellt werden.

\subsubsection{Operante Faktoren, Angst vor Schmerz und Vermeidung}

Bei der Betrachtung der psychologischen Seite des bio-psycho-sozialen Modells wird nicht nur das Schmerzerleben in seinem Einfluss auf die Entwicklung einer Schmerzstörung in den Blick genommen, sondern auch das Schmerzverhalten. Wie die Theorie des operanten Konditionierens vorhersagt, wird Verhalten dann aufrechterhalten, wenn es positiv oder negativ verstärkt wird. Führt z.B. Tabletteneinnahme zu einer 
Schmerzreduktion, ohne dass deutlich wahrnehmbare negative Konsequenzen auftreten, wird dies Verhalten negativ verstärkt und dadurch häufiger gezeigt. Wenn ein Patient insbesondere auf Schmerzäußerungen liebevolle Zuwendung erhält oder von unangenehmen Pflichten befreit wird, kann dies dazu führen, dass der Patient verstärkt Schmerz äußert, vor allem, wenn er auf anderem Wege wenig Aufmerksamkeit erhält. Besonders Fordyce (1976) hat die Rolle solcher Lernprozesse für die Aufrechterhaltung von Schmerzverhalten betont. Den Einfluss zuwendenden Verhaltens von Ehepartnern auf das Verhalten von Schmerzpatienten zeigen auch Lousberg, Schmidt und Groenman (1992). Sie untersuchten Patienten mit einem Partner, der liebevoll auf Schmerzäußerungen reagierte. Die Patienten äußerten in Anwesenheit ihres Partners mehr Schmerzen und zeigten bei einer körperlichen Aufgabe geringere Leistungen, als wenn der Partner nicht anwesend war. Schmerzäußerung und Vermeidungsverhalten wurden hier offenbar durch die liebevolle Zuwendung des Partners verstärkt. Dass das Schmerzverhalten eng mit dem Schmerzerleben verbunden ist, wird in einer Untersuchung zu Schmerzverhalten und Schmerzschwelle von Flor, Breitenstein, Birbaumer und Fürst (1995) deutlich. Je unterstützender und besorgter sich die Ehepartner der Patienten zeigten, desto geringer war die Schmerzschwelle der Patienten. Die liebevolle Unterstützung der Ehepartner förderte offenbar die Aufmerksamkeit der Patienten auf den Schmerz, der dadurch verstärkt wahrgenommen wurde.

Philips (1987) setzt in ihrem Modell des Avoidance-Lernens einen anderen Schwerpunkt. Anders als Fordyce geht sie davon aus, dass die "secondary losses", die ein Patient durch seine Schmerzen erleidet (Kontaktverlust, Arbeitsverlust) die "secondary gains" bei weitem übersteigen, dass das Konzept von Fordyce also keine ausreichende Erklärung für Chronifizierung liefert. Sie legt den Schwerpunkt der Betrachtung auf das Vermeidungsverhalten der Patienten. Vermeidungs- und Schonverhalten bei akutem Schmerz ist ein biologisch gebahntes, sinnvolles Verhalten, das zudem, wenn es zu Schmerzlinderung führt, negativ verstärkt wird. Wird dieses Schonverhalten nun aber über längere Zeit aufrechterhalten, kann dies zu negativen körperlichen Folgen führen, wie z.B. zu Muskelabbau und genereller Verschlechterung des körperlichen Zustands, aber auch zur Inaktivität körpereigenener schmerzhemmender Systeme (z.B. Endorphine), die durch körperliche Betätigung angeregt werden. Darüber hinaus kann die Vermeidung von Aktivitäten und Rückzug auch zu sozialer Isolation führen und damit zu einem Verlust an positiven Verstärkern, der wiederum zu Depressivität führen kann. Sowohl die oben beschriebene Verschlechterung des körperlichen Zustandes als auch die 
Depressivität können das Schmerzerleben verstärken, worauf der Patient möglicherweise mit noch mehr Schonung und Rückzug reagiert. Diesen Teufelskreis sieht Philips als einen wesentlichen Faktor bei der Chronifizierung von Schmerzen an.

Im chronischen Zustand führt also Vermeidungs- und Rückzugsverhalten zumeist nicht mehr zu dem erwünschten Erfolg der Schmerzlinderung. Interessant ist die Frage, warum dieses Verhalten, das den erwarteten Erfolg nicht nach sich zieht, dennoch aufrechterhalten wird. Die Überlegungen von Melzak and Wall (1982 zit. nach Philips 1987) im Rahmen ihrer Gate-Control-Theorie, dass das Vermeidungsverhalten dadurch gesteuert wird, wie Patienten den Schmerz affektiv bewerten (z.B. als lähmend oder entnervend), weist Philips in diesem Zusammenhang zurück, da sie in verschiedenen Untersuchungen zeigen konnte, dass sich Menschen mit geringem Vermeidungsverhalten von solchen mit starkem Vermeidungsdrang nicht in der Affektkomponente der Schmerzbewertung unterscheiden (Philips 1987). Philips geht davon aus, dass als wesentlicher Faktor zur Aufrechterhaltung des Vermeidungsverhaltens kognitive Prozesse eine Rolle spielen.

Unter anderem wurde in diesem Zusammenhang die Rolle der Schmerzerwartung diskutiert. Zunächst wurde davon ausgegangen, dass die Schmerzerwartung unmittelbar das Schmerzerleben bestimmt: je höher die Schmerzerwartung desto höher der erlebte Schmerz (Pennebaker 1982). Crombez, Eccleston, Baeyens und Eelen (1996) zeigen jedoch, dass Menschen auch bei hoher Schmerzerwartung nicht unbedingt starken Schmerz erleben, sie jedoch bei höherer Schmerzerwartung eine stärkere Neigung zur Vermeidung zeigen. Crombez et al. gehen davon aus, dass die Erwartung von Schmerz nicht den Schmerz selbst hervorruft oder verstärkt, sondern die Angst vor Schmerz, was dazu führt, dass die Menschen versuchen, Schmerz zu vermeiden. Das würde erklären, warum Vermeidungsverhalten auch dann aufrechterhalten wird, wenn es nicht zu Schmerzlinderung führt: nicht der Schmerz wird beseitigt, sondern die Angst vor Schmerz und durch diesen Mechanismus wird das Vermeidungsverhalten negativ verstärkt.

Die Bedeutung von Angst vor Schmerzen betonen auch Lethem, Slade, Troup und Bentley (1983) in ihrem Fear-Avoidance-Modell. Sie gehen davon aus, dass Angst vor Schmerzen im Zusammenhang mit Schmerzerleben eine wesentlich Rolle spielt. Ihnen zufolge haben Menschen, die Schmerzen erleben, vor allem zwei Möglichkeiten, mit der Angst vor Schmerzen umzugehen: Konfrontation oder Vermeidung. Welche dieser beiden Möglichkeiten sie wählen, hängt von verschiedenen Faktoren ab, wie der Anzahl 
der erlebten Life events, der persönlichen Schmerzgeschichte, den bevorzugten Copingstrategien und von Persönlichkeitsfaktoren.

Um der Frage nachzugehen, welche Patienten eher zu Angst vor Schmerzen und zu Vermeidungsverhalten neigen, verweisen Asmundson und Norton (1995) auf das Konzept der Anxiety Sensitivity (Reiss, Peterson, Gursky \& McNally 1986, Reiss, Peterson \& Gursky 1988, Cox, Parker \& Swinson 1996). Entwickelt wurde dies Konzept im Zusammenhang mit Angststörungen, insbesondere Panikstörungen. Dabei geht es um Überzeugungen über die Schädlichkeit angstbezogener Körperempfindungen. Je eher eine Person der Überzeugung ist, dass angstbezogene Körperreaktionen, wie z.B. Herzrasen, ihr schaden könnten, desto mehr Aufmerksamkeit wird sie diesen widmen, bzw. sie wird versuchen, Angstgefühle generell zu vermeiden. Diese Überzeugung und das daraus resultierende Verhalten kann die Entwicklung einer Panikstörung begünstigen. Asmundson und Norton (1995) stellten die Hypothese auf, dass Schmerzpatienten mit einer erhöhten Anxiety Sensitivity größere Angst vor Schmerzen haben und mehr Vermeidungsverhalten zeigen als Patienten mit geringerer Anxiety Sensitivity. Dies konnten sie in ihrer Studie bestätigen. Asmundson und Taylor (1996) konnten mit Hilfe einer Pfadanalyse darstellen, dass es bei Patienten mit muskeloskeletalen Schmerzen einen engen Zusammenhang zwischen Anxiety Sensitivity, Angst vor Schmerzen und Vermeidungsverhalten gibt.

Darüber hinaus wurde in einer Studie von Asmundson, Kuperos und Norton (1997) deutlich, dass Patienten mit muskeloskeletalen Schmerzen, die eine erhöhte Anxiety Sensitivity aufweisen, eher verstärkt Aufmerksamkeit auf schmerzrelevante Stimuli richten, während Patienten mit niedriger Anxiety Sensitivity diese eher ignorieren.

Mit der Diskussion operanter Faktoren des Schmerzverhaltens und der Bedeutung von Vermeidungs-und Schonverhalten im Zusammenhang mit Angst vor Schmerzen sind Prozesse angesprochen, die zur Erklärung der Chronifizierung bzw. Aufrechterhaltung von Schmerzstörungen im allgemeinen herangezogen werden. Im folgenden soll nun auf einen Aspekt näher eingegangen werden, der insbesondere im Zusammenhang mit chronischen Rückenschmerzen diskutiert wird, die Angst vor Bewegungen. 


\subsubsection{Bewegungsangst und Fear-Avoidance Beliefs}

Bei Menschen mit chronischen Rückenschmerzen könnte neben der allgemeinen Angst vor Schmerzen eine Angst vor bestimmten Bewegungen eine Rolle spielen, die als Schmerz auslösend erfahren worden sind. Diese Überlegung greifen Kori, Miller und Todd (1990) bei der Entwicklung eines Modells der Kinesiophobie auf. Die Mechanismen des Erwerbs und der Aufrechterhaltung einer solchen Kinesiophobie entsprechen denen anderer Phobien, bei denen das Zwei-Faktoren-Modell von Mowrer (1939) eine bedeutsamen Rolle spielt. Kori et al. gehen Bezug nehmend auf dieses Modell davon aus, dass bei vielen Rückenschmerzpatienten bestimmte Bewegungen anfänglich starken Schmerz verursachen, so dass die Angst vor der Bewegung zunächst klassisch konditioniert wird. Die Bewegung wird zukünftig eher vermieden, durch die Vermeidung wird die Angst reduziert und das Vermeidungsverhalten negativ verstärkt (operante Konditionierung). Menschen mit Angst vor Bewegungen vermeiden also im Alltag vielerlei Aktivitäten. Die Folgen solchen Vermeidungsverhaltens sind die gleichen wie Philips sie beschreibt - immer stärkerer Rückzug im Alltag, sowohl von körperlicher Betätigung, was zu Muskelabbau bzw. genereller Verschlechterung des körperlichen Zustands führen kann, als auch Rückzug aus sozialen Netzen, was gegebenenfalls Depressivität hervorruft. Der Verbindung zwischen Angst vor Bewegungen, Vermeidung und Beeinträchtigung im Alltag sind unter anderen Vlaeyen, Kole-Snijders, Boeren und van Eek (1995) und Crombez, Vlaeyen, Heuts und Lysens (1999) nachgegangen. Sie konnten zeigen, dass Patienten mit hoher Angst vor Bewegungen (gemessen mit der Tampa Scale of Kinesiophobia -TSK -Miller, 1991 zitiert nach Vlaeyen et al. 1995) geringere Bewegungsleistungen im Experiment zeigten und im Alltag von mehr Beeinträchtigungen berichteten als Patienten, die weniger Angst vor Bewegungen äußerten. McCracken, Gross, Sorg und Edmans (1993) zeigten, dass Patienten, die erwarteten, dass eine bestimmte Bewegung Schmerz auslösen würde, bei einer Bewegungsaufgabe weniger Leistung zeigten, als Patienten mit geringerer Schmerzerwartung und zwar unabhängig von dem Bericht über die tatsächlichen Schmerzen. In einer Untersuchung von Pfingsten, Kröner-Herwig, Hempel, Harter und Hildebrandt (1999) wurde die Schmerzerwartung von Patienten experimentell induziert, indem einer Gruppe von Patienten suggeriert wurde, dass eine auszuführende Bewegungsaufgabe schmerzhaft sein würde. Diese Gruppe zeigte eine deutlich höhere Schmerzerwartung und bei der nachfolgenden Bewegungsaufgabe eine stärkere Neigung 
zur Vermeidung als Patienten, denen die Bewegungsaufgabe als nicht Schmerz auslösend angekündigt war. Es scheint also so zu sein, dass die Erwartung darüber, ob eine bestimmte Aktivität Schmerz auslösen oder verstärken wird, das Verhalten der Person stark beeinflusst und somit letztendlich das Ausmaß der erlebten Beeinträchtigung. Diesem Gedanken folgen auch Waddell et al. (1993). Sie gehen davon aus, dass einige Rückenschmerzpatienten überdauernde Überzeugungen entwickeln über den Zusammenhang von bestimmten Bewegungen und Aktivitäten und ihren Schmerzen. Diese überdauernden Überzeugungen steuern ihr alltägliches Handeln: Je eher eine Person der Ansicht ist, dass bestimmte Bewegungen oder Handlungen den Schmerz verstärken werden, desto eher wird sie diese vermeiden und sich beeinträchtigt fühlen. Solche rückenschmerzspezifischen Annahmen des Patienten bezeichnen Waddell et al. als "Fear-Avoidance Beliefs". Entscheidend dabei ist, dass es sich um überdauernde Überzeugungen über den Zusammenhang zwischen bestimmten Aktivitäten und Rückenschmerzen handelt.

Die Bedeutsamkeit solcher Fear-Avoidance Beliefs für den Verlauf von Rückenschmerzerkrankungen konnte mittlerweile in mehreren Studien deutlich gemacht werden. Erfasst werden diese Überzeugungen mit Hilfe des von Waddell et al. (1993) entwickelten Fear-Avoidance Beliefs Questionnaire. Dieser enthält Items zum Zusammenhang zwischen Rückenschmerz und alltäglichen Aktivitäten ebenso wie den Zusammenhang zur Arbeit. Die Ergebnisse dieses Fragebogens konnten bei einer Untersuchung zur Entwicklung von Beeinträchtigung und Arbeitsverlust einen erheblichen Anteil an Varianz aufklären (Waddell et al. 1993). Auch Pfingsten, KrönerHerwig, Leibing, Kronshage und Hildebrandt (2000) berichten ähnliche Ergebnisse in einer deutschen Studie; der Anteil an Varianzaufklärung für die Vorhersage von Arbeitsunfähigkeit und Beeinträchtigung im täglichen Leben lag hier sogar noch höher. In der oben beschriebenen Untersuchung von Pfingsten et al. (1999) zur Bewegungsleistung im Experiment mit induzierter Schmerzerwartung zeigte sich, dass Patienten mit starken Fear-Avoidance Beliefs stärker zu Vermeidung neigten als die mit geringer ausgeprägten Beliefs. Klenerman, Slade, Stanley, Pennie, Reilly und Atchison (1995) fanden bei einer Längsschnittuntersuchung akut erkrankter Rückenschmerzpatienten, dass Personen, die im akuten Stadium von Rückenschmerzen erhöhte Fear-Avoidance Beliefs zeigten, eine erhöhte Wahrscheinlichkeit aufweisen, dass der Rückenschmerz chronifiziert. Die Bedeutsamkeit von Fear-Avoidance Beliefs für die 
Vorhersage von Arbeitsunfähigkeit und zusätzlich für Hilfesuchverhalten (Arztkontakte) zeigten auch McCracken, Gross, Aikens und Carnike (1996).

Der Zusammenhang von Fear-Avoidance Beliefs und Vermeidungsverhalten bzw. Beeinträchtigung hat sich also wiederholt zeigen lassen. Auch das Konzept der Bewegungsangst stellt ein generelles Rückszugs-und Schonverhalten und das Gefühl der Beeinträchtigung als entscheidend in den Vordergrund. Da gezeigt werden konnte, dass bei dem Prozess der Chronifzierung von Rückenschmerzen nicht die zugrunde liegende Pathologie und auch nicht die Schmerzstärke entscheidend sind, sondern das Gefühl der Beeinträchtigung, erscheinen beide Konzepte in diesem Zusammenhang bedeutsam. Der Zusammenhang zwischen den Konzepten der Kinesiophobie und der Fear-Avoidance Beliefs ist jedoch noch unklar. So ist es nicht sicher, ob Menschen mit ausgeprägten Fear-Avoidance Beliefs tatsächlich Angst vor Bewegungen erleben oder ob diese Überzeugungen eher kognitive Konzepte sind, die das Verhalten beeinflussen, ohne dass ein Gefühl von Angst vorhanden ist.

Grundsätzlich wäre es möglich, dass Patienten Fear-Avoidance Beliefs entwickeln, ohne vorher eine Bewegungsangst auszuprägen, dass die beiden Konzepte also voneinander unabhängig sind. Andererseits könnten sich Fear-Avoidance Beliefs auch aus einer erlebten Angst vor Bewegungen herausbilden und eine Art Trait-Komponente der Angst vor Bewegungen darstellen. Abbildung 2.1 zeigt in einem erweiterten Modell von Waddell et al. (1993) die möglichen Einflüsse der beiden Konzepte auf die Beeinträchtigung, bei der die beiden Konzepte als eher unabhängig angesehen werden. 


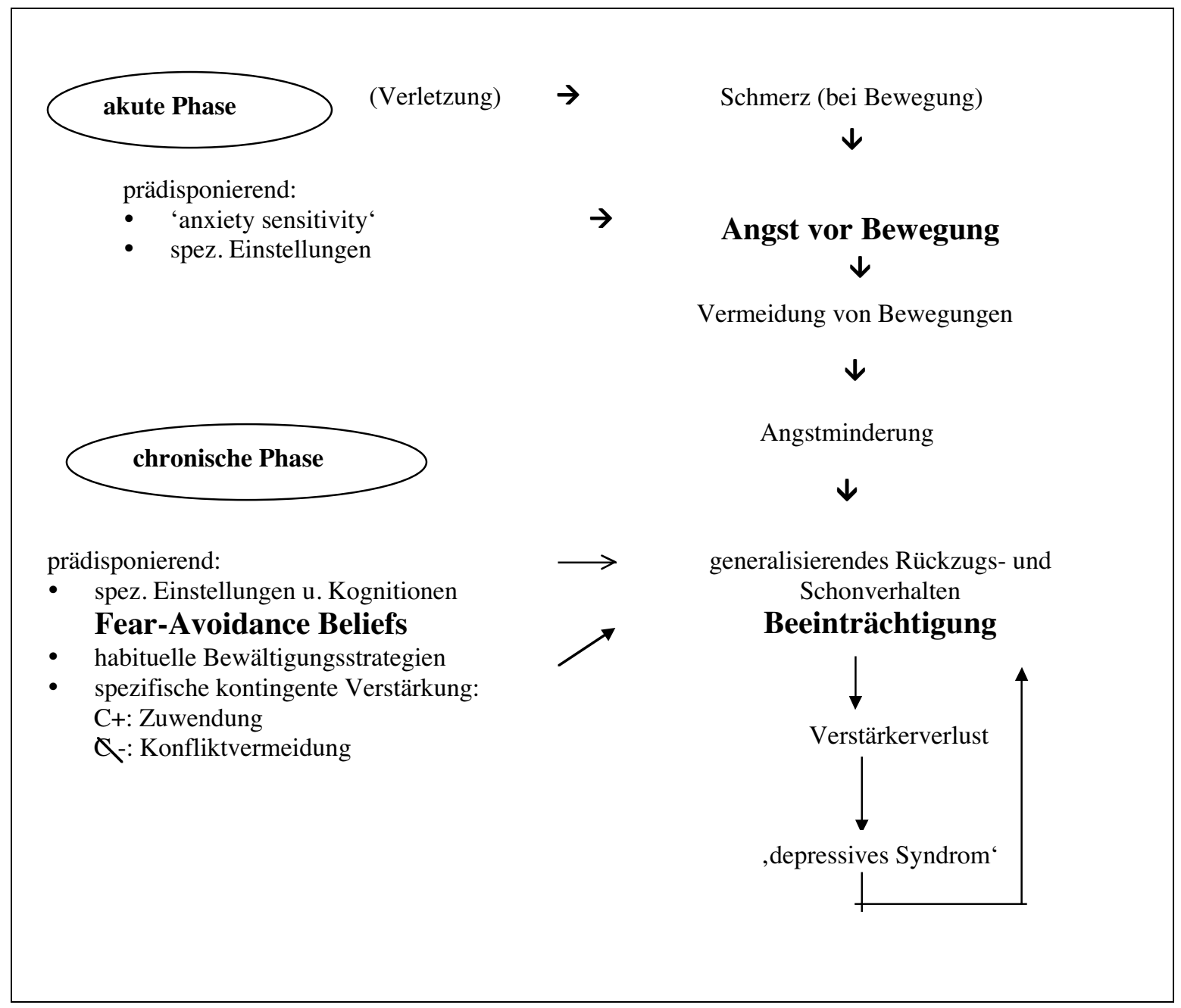

Abbildung 2.1 Modell der Chronifizierung von Rückenschmerzen (nach Kröner-Herwig, 2000)

\subsection{Untersuchung der Konzepte Bewegungsangst und Fear-Avoidance Beliefs}

Da die Konzepte der Bewegungsangst und der Fear-Avoidance Beliefs im Zusammenhang mit der Chronifizierung von Rückenschmerzen möglicherweise eine entscheidende Rolle spielen, sollen sie in der vorliegenden Studie genauer untersucht werden. Bei Studien zur Bewegungsangst wurden bisher meist Fragebogendaten erhoben, also subjektive Angaben über die von den Probanden erlebte Angst (s. Vlaeyen und Linton, 2000). Der Fear-Avoidance Beliefs Questionnaire hat sich zwar als Prädiktor für verschiedene Beeinträchtigungsaspekte in mehreren Studien bewährt, noch ist jedoch nicht geklärt, was dieser Fragebogen eigentlich erfasst. Wie weiter oben bereits beschrieben, ist es unklar, ob Patienten mit ausgeprägten Fear-Avoidance Beliefs tatsächlich Angst vor Bewegungen erleben, oder ob es sich eher um kognitive Schemata 
zur Schädlichkeit von Bewegungen handelt, die relativ unabhängig von Angst vor Bewegungen auftreten. Darüber hinaus wird von Waddell et al. (1993) diskutiert, dass Fear-Avoidance Beliefs möglicherweise einen engen Zusammenhang zur Arbeitszufriedenheit zeigen.

Um die Konzepte Bewegungsangst und Fear-Avoidance Beliefs genauer zu beleuchten, soll zunächst im Experiment überprüft werden, ob Patienten mit chronischen Rückenschmerzen bei der Konfrontation mit rückenschmerzrelevanten Bildern Angst vor Bewegungen zeigen. Dafür sollen zwei Paradigmen verwendet werden, die sich im Zusammenhang mit der Untersuchung von Angst und Phobien bereits mehrfach bewährt haben: das Startle-Paradigma und das Paradigma des Covariation Bias. Beide Verfahren bieten gegenüber Fragebogendaten den Vorteil, dass die Reaktion von den Probanden nicht bewusst gesteuert werden kann. Die Ableitung der Startle-Reaktion (Schreckreaktion) dient als physiologisches Maß für einen eventuellen Angstzustand der Probanden, wohingegen der Covariation Bias Rückschlüsse darauf erlaubt, wie bedrohlich ein Reiz von einer Person wahrgenommen wird. Beide Verfahren werden im Folgenden ausführlicher dargestellt.

Zusätzlich zu der Untersuchung der Patienten mit den beiden erwähnten Methoden soll die Frage geklärt werden, ob Patienten mit ausgeprägten Fear-Avoidance Beliefs mehr Angst vor Bewegungen im Experiment zeigen als Patienten mit weniger ausgeprägten Beliefs. Dies könnte als ein Hinweis gewertet werden, dass die beiden Konzepte in Zusammenhang stehen. Um das Konstrukt der Fear-Avoidance Beliefs darüber hinaus noch genauer zu beleuchten, werden zwei eigene Fragebögen entwickelt, die mögliche Aspekte von Fear-Avoidance Beliefs abbilden. Ein Fragebogen soll dabei subjektiv erlebte Angst beim Auftreten von Schmerzen messen und der andere allgemeinere Einstellungen zum Zusammenhang zwischen Schmerz und Aktivitäten. Um den Zusammenhang zu Arbeitszufriedenheit untersuchen zu können, wird der Arbeitsbeschreibungsbogen (ABB) von Neuberger und Allerbeck (1978) eingesetzt.

Zunächst sollen nun die beiden Paradigmen vorgestellt werden, die zur Überprüfung der Angstrelevanz von Bewegungen eingesetzt werden sollen und danach die Fragebögen zur genaueren Untersuchung des Konzepts der Fear-Avoidance Beliefs. 
2.3.1 Untersuchung der Konzepte Bewegungsangst und Fear-Avoidance Beliefs unter Verwendung experimenteller Paradigmen

\section{- Das Startle-Paradigma}

Zur Untersuchung der emotionalen Qualität eines Stimulus kann der Schreckreflex (Startle-Reflex) am Auge herangezogen werden (Vrana, Spence und Lang, 1988). Der Schreckreflex ist ein Ganzkörperreflex, der nach plötzlichen, intensiven und unerwarteten Stimuli, etwa einem lauten Ton oder einem hellen Lichtblitz, auftritt. Als erstes und deutlichstes Zeichen dieses Schreckreflexes zeigt sich das schnelle Schließen der Augenlider. Gemessen wird der Reflex üblicherweise an der Höhe des EMG des Orbicularis Oculi (s. Abb. 2.2).

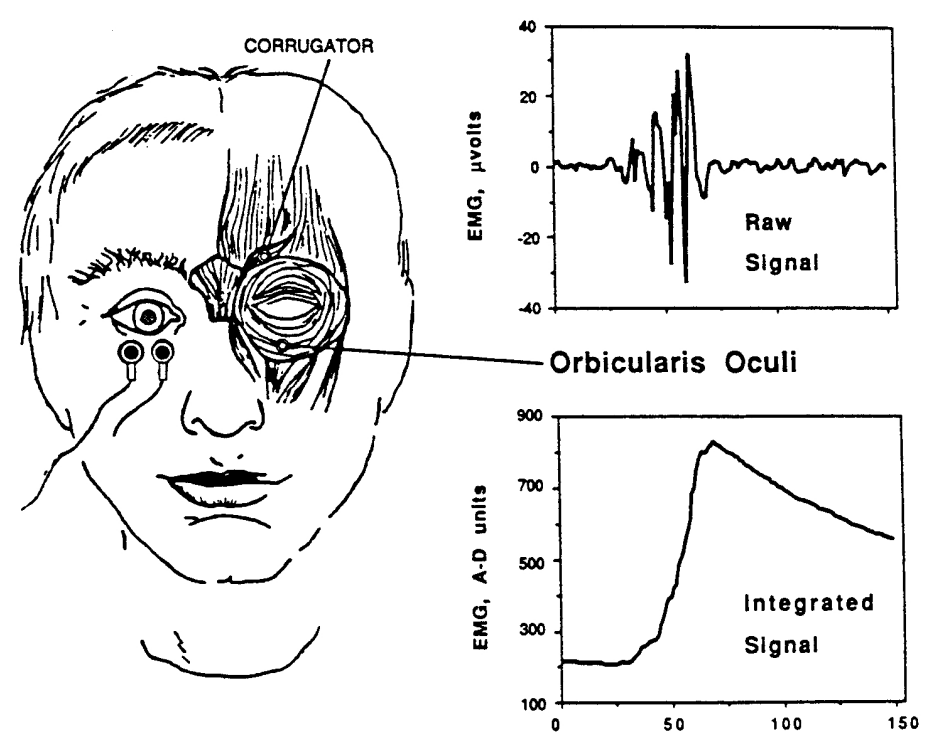

Abbildung 2.2: Ableitung des Schreckreflexes am Orbicularis Oculi, Darstellung der Rohwertegraphik (rechts oben) und des Integrierten Signals (rechts unten), nach Lang, Bradley und Cuthbert (1990)

Die Stärke des Schreckreflexes ist abhängig von der emotionalen Situation, in der sich eine Person befindet. Wie Lang, Bradley und Cuthbert (1990) beschreiben, basiert das emotionale System auf zwei grundlegenden motivationalen Zuständen: der Annäherung an eine Situation oder der Vermeidung. Reflexe können dadurch gekennzeichnet werden, ob sie eher der Annäherungs- oder der Vermeidungsdimension zuzuschreiben sind. Da beim Schreckreflex das motivationale System der Vermeidung/Flucht aktiviert ist, wird 
dieser Reflex in einer emotional aversiven Situation im Vergleich zu einer neutralen Situation verstärkt, während er in einer positiven emotionalen Situation eher gehemmt wird.

Der Startle-Reflex reflektiert also nicht die Emotion direkt, sondern gibt Aufschluss über den zugrunde liegenden motivationalen Zustand des Systems. Tierexperimente (Davis, Walker und Lee, 1999) und Einzelfallstudien am Menschen (Angrilli, Mouri, Palomba, Flor, Birbaumer et al.,1996) zeigen an, dass beim Startle-Reflex subcorticale Strukturen, insbesondere die Amygdala, Ausschlag gebend beteiligt sind. Die Stärke des Reflexes ist durch bewusste Steuerung nicht beeinflussbar, Aspekte sozial erwünschten Verhaltens spielen also keine Rolle, was einen Vorteil gegenüber Fragebogendaten bedeutet.

Bei der Untersuchung des Einflusses des emotionalen Hintergrundes auf die StartleReaktion werden zur Induktion von Emotionen üblicherweise visuelle Stimuli eingesetzt, es gibt aber auch Studien, die Vorstellungsübungen oder Geschichten verwenden (Vrana und Lang, 1990). Als visuelle Stimuli werden in der Regel die Bilder des International Affective Picture Systems genutzt (Lang, Bradley und Cuthbert, 1997). Es beinhaltet über 600 Bilder, die auf den Dimensionen emotionale Valenz und Erregung an großen Gruppen von Probanden normiert worden sind. Normen existieren sowohl für Frauen und Männer getrennt als auch für beide Gruppen gemeinsam. Mit diesen Bildern ist es möglich, zuverlässig emotionale Reaktionen von Anziehung (Freude, Zärtlichkeit, erotische Anziehung) und Aversion (Trauer, Ekel, Angst) ebenso wie neutrale Reaktionen hervorzurufen. Bei der Betrachtung der aversiven Bilder zeigt sich eine deutliche Erhöhung der Startle-Reaktion gegenüber der Reaktion auf neutrale Bilder, während die Startle-Reaktion auf positive Bilder gegenüber der auf neutrale Bilder gehemmt wird (s. Abb. 2.3). 


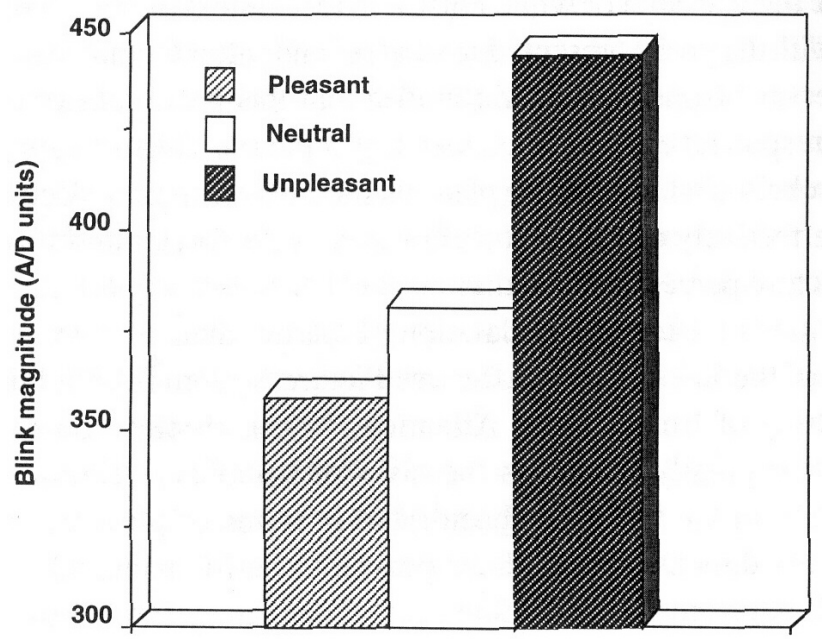

Abbildung 2.3 Startle-Reaktion auf positive, negative und neutrale Bilder (nach Bradley, Cuthbert und Lang, 1999)

Nicht nur unterschiedliche Startle-Reaktionen auf diese Bilder sind nachgewiesen worden, sondern auch unterschiedliche Reaktionen in Bezug auf die Herzrate, den Blutdruck und die Hautleitfähigkeit (Lang, Greenwald, Bradley und Hamm, 1993). Diese physiologischen Parameter zeigen jedoch nur das generelle Erregungsniveau, während die Startle-Reaktion ein zuverlässiges Maß darstellt, um die emotionale Qualität der Situationen zu unterscheiden, das heißt, positive von negativer Erregung abzugrenzen.

Um eine deutliche Startle-Reaktion zu bekommen, scheint es allerdings notwendig zu sein, dass die Emotionsinduktion eine ausreichend hohe Erregung (Arousal) hervorruft. In vielen Untersuchungen zeigte sich, dass eine klare Unterscheidung von positiven und negativen Bildern aufgrund der Startle-Reaktion nur möglich ist, wenn der Arousal-Wert der Bilder von den Versuchspersonen als ausreichend stark eingeschätzt wird. Cuthbert, Bradley und Lang (1996) gingen dieser Frage in einer Untersuchung systematisch nach und kamen zu dem Ergebnis, dass der Arousal-Wert auf einer 9-stufigen Skala (1= schwache Erregung, 9 = sehr starke Erregung) bei mindestens sechs liegen sollte, damit ein Unterschied in der Startle-Reaktion zuverlässig nachweisbar ist.

Dass der Startle-Reflex auch sensibel auf Furcht-Konditionierungsprozesse reagiert, berichten Hamm und Vaitl (1996). Werden ursprünglich neutrale Stimuli mit einem aversiven Stimulus (z.B. Hitzereiz) gepaart, zeigt sich bei der Konfrontation mit dem konditionierten Reiz eine deutliche Erhöhung des Startle-Reflexes. Entsprechend zeigt 
sich bei Menschen mit Phobien eine Erhöhung des Startle-Reflexes im Vergleich zu Kontrollpersonen, wenn sie mit ihren spezifischen Angstreizen (z.B. mit Bildern von Hunden oder Schlangen) konfrontiert werden (Hamm, Cuthbert, Globisch und Vaitl, 1997). Werden phobische Patienten therapeutisch behandelt und verringert sich nachfolgend ihre Angst vor bestimmten Stimuli, zeigt sich dies in einer Verminderung des Startle-Reflexes im Vergleich zu der Zeit vor der Therapie (DeJong, Merckelbach und Arntz, 1991). Die Erhöhung des Startle-Reflexes scheint aber auch von generellen Persönlichkeitsmerkmalen abzuhängen, insbesondere von der allgemeinen Ängstlichkeit. Cook, Hawk, Davis und Stevenson (1991) konnten zeigen, dass die Erhöhung der StartleReaktion bei der Konfrontation mit unangenehmen Reizen bei hoch ängstlichen Personen (gemessen mit dem Fear Survey Schedule) deutlich stärker ist als bei niedrig ängstlichen Personen und zwar unabhängig davon, ob es sich bei den negativen Stimuli eher um Angst erregende, traurige oder Ekel erregende Reize handelte. Ähnliche Ergebnisse berichten auch Grillon, Rezvan, Woods, Merikangas und Davis (1991).

Der Startle-Reflex wird jedoch nicht nur von der emotionalen Situation bestimmt, in der sich die Person befindet, sondern auch von Aufmerksamkeitsprozessen (Anthony \& Graham, 1985). Wird vor dem Reiz, der die Startle-Reaktion auslöst, ein interessanter Reiz in der gleichen Sinnesmodalität wie der Startle-Reiz dargeboten, wird die StartleReaktion verstärkt (ein interessantes Musikstück kurz vor einem akustischen Startle-Reiz verstärkt also die Startle-Reaktion). Wird der vorgeschaltete Reiz in einer anderen Sinnesmodalität dargeboten, wird die Startle-Reaktion gehemmt. Je mehr Aufmerksamkeit also auf die Modalität gelenkt wird, in der der Startle-Reiz dargeboten wird, desto größer ist die Reaktion. Obwohl Bradley, Cuthbert und Lang (1990) aufgrund ihrer Untersuchungen zur Bedeutsamkeit von Aufmerksamkeitsprozessen und emotionalem Hintergrund für die Modulation der Startle-Reaktion zu dem Schluss kommen, dass der emotionale Hintergrund einer Startle-Reaktion entscheidender ist als Aufmerksamkeitsprozesse, zeigten einige andere Studien ein eher heterogenes Bild, so dass noch nicht abschließend festzustellen ist, ob und unter welchen Umständen einer der beiden Prozesse eher wirksam wird (für eine Übersicht über die Studien siehe Filion, Dawson und Schell, 1998 und Putnam und Vanman, 1999). Auch über die Länge des Zeitintervalls, das zwischen vorgeschaltetem Reiz und Startle-Reiz liegen muss, damit Aufmerksamkeits- bzw. emotionale Prozesse sich auswirken können, gibt es unterschiedliche Angaben. Während zunächst davon ausgegangen wurde, dass eine Modulation der Startle-Reaktion durch den emotionalen Hintergrund nur bei so 
genannten „Long-Lead-Intervallen“ von mehr als $800 \mathrm{~ms}$ stattfinden, während Aufmerksamkeitsprozesse schon bei kürzeren Intervallen eine Rolle spielen können, zeigen neuere Untersuchungen auch Einflüsse des emotionalen Hintergrunds bei kürzeren Intervallen (250 und 750 ms, Vanman, Böhmelt, Dawson und Schell, 1996). Da diese Ergebnisse noch nicht eindeutig sind, erscheint es zur Zeit sinnvoll, bei Studien zur Bedeutung des emotionalen Hintergrunds der Startle-Reaktion eher Long-Lead-Intervalle zu nutzen.

Zusammenfassend lässt sich sagen, dass die Startle-Reaktion ein physiologisches Maß ist, mit dem sich ein Zustand von Angst zuverlässig nachweisen lässt. Geht man also davon aus, dass es bei chronischen Rückenschmerzpatienten eine konditionierte Angst vor Bewegungen gibt, so sollte sich dies in einer Erhöhung der Startle-Reaktion widerspiegeln, wenn die Patienten mit rückenschmerzrelevanten Bildern konfrontiert werden. Darüber hinaus ist bekannt, dass generelle Ängstlichkeit ebenfalls einen Einfluss auf die Startle-Reaktion hat. Wenn also Fear-Avoidance Beliefs in Bezug auf Bewegungsangst eine Art Trait-Angst widerspiegeln, so sollte sich das ebenfalls in der Startle-Reaktion zeigen: Personen mit ausgeprägteren Fear-Avoidance Beliefs sollten dann eine deutlichere Erhöhung der Reaktion zeigen, wenn sie mit rückenschmerzrelevanten Bildern konfrontiert werden.

\section{- Der Covariation Bias}

Während das Startle-Paradigma Rückschlüsse auf den emotional-motivationalen Zustand einer Person bzw. des Organismus zulässt, werden mit dem Paradigma des Covariation Bias kognitive Prozesse der Informationsverarbeitung untersucht. Ausgehend von Erkenntnissen über Erinnerungsfehler von Depressiven, die dazu neigen, sich an unangenehme Ereignisse besser zu erinnern als an angenehme (Blanley, 1986), wurde die Frage aufgeworfen, ob Personen mit Phobien möglicherweise die Verbindung von angstrelevanten Stimuli mit aversiven Ereignissen überschätzen und so zu einem Fehlurteil über die Gefährlichkeit der Reize kommen. Um dieser Frage nachzugehen entwickelten Tomarken, Mineka und Cook (1989) einen Versuchsaufbau, der im folgenden beschrieben werden soll. Personen mit spezifischen Phobien (z.B. Schlangen oder Spinnen) bekamen ebenso wie nicht ängstliche Kontrollpersonen Bilder von angstrelevanten, neutralen bzw. angenehmen Stimuli zu sehen. In einem Drittel aller Fälle wurden die Bilder jeder Kategorie (angstbesetzt, neutral, angenehm) mit einem 
aversiven Reiz (Schock) verbunden präsentiert. Nach der Bilderpräsentation sollten die Versuchspersonen angeben, welche Bilder zusammen mit dem aversiven Reiz dargeboten worden waren. Als Ergebnis zeigte sich, dass beide Gruppen die Häufigkeit der Assoziation zwischen angstrelevantem Reiz und dem Schock überschätzten (Covariation Bias), was bei den neutralen und den positiven Bildern nicht der Fall war. Bei der Gruppe der phobischen Versuchspersonen war diese Überschätzung jedoch deutlich ausgeprägter als bei der Gruppe der nicht ängstlichen Versuchspersonen. Es scheint also eine generelle Tendenz zu geben, bei unangenehmen Bildern die Verbindung mit aversiven Reizen zu überschätzen, wobei phobische Personen diese Tendenz stärker zeigen. Unterziehen sich phobische Patienten einer verhaltenstherapeutischen Behandlung, so verringert sich bei erfolgreicher Therapie das Ausmaß des Covariation Bias und gleicht sich dem von nicht ängstlichen Personen an (deJong und Merckelbach, 1991). Wird die Häufigkeit der Assoziation eines potentiell angstrelevanten Reizes (wie z.B. ein Bild von einer Schlange oder einem großen Hund) mit einem aversiven Reiz stark erhöht (z.B. auf 80\%), so zeigen auch niedrig ängstliche Personen einen Covariation Bias, der denen phobischer Personen entspricht (Pauli, Montoya und Martz 1996). Erfahrungen von Kontingenzen zwischen dem angstrelevanten Reiz und aversiven Reizen führen also offenbar zur Ausbildung eines Schemas über die Gefährlichkeit eines Reizes, welches dann die Erinnerung an Kontingenzen steuert. Wird ein Stimulus sehr oft mit einem aversiven Stimulus gemeinsam erlebt, so beeinflusst diese Erfahrung die Einschätzung von Kontingenzen aus der Erinnerung. Interessanterweise ist der Covariation Bias, also eine falsche Einschätzung im nachhinein, wesentlich stärker abhängig vom vorherigen Angstlevel und wird durch experimentelle Erfahrung von geringen Kontingenzen wesentlich weniger beeinflusst als ein entsprechender Erwartungsbias (also eine falsche Prognose über Kontingenzen, Davey und Dixon, 1996). Die Erwartung einer Kontingenz von angstrelevantem und aversivem Reiz ist anders als beim Covariation Bias zumeist bei hoch ängstlichen und niedrig ängstlichen Personen gleich und wird durch Erfahrung im Experiment relativ schnell den tatsächlichen Wahrscheinlichkeiten der Kontingenz angeglichen. Wenn also im Experiment zunächst viele Kontingenzen erfolgen, werden auch viele Kontingenzen erwartet. Verringern sich die Kontingenzen jedoch, dann erwarten die Personen auch geringere Kontingenzen und zwar unabhängig von ihrem generellen Angstlevel. Der Covariation Bias hingegen ist, wie oben beschrieben, bei hoch ängstlichen Personen deutlich höher und wird nicht durch Erfahrungen im Experiment beeinflusst. Verringert 
sich die Assoziationshäufigkeit im Experiment, wird der Covariation Bias nicht korrigert (Amin und Lovibond, 1997). Mit Hilfe des Covariation Bias ist es im Gegensatz zum Erwartungsbias also möglich, hoch ängstliche Personen von niedrig ängstlichen Personen zu unterscheiden. Wenn eine Person einen Stimulus als angstauslösend erlebt, dann wird sie dazu neigen, die gemeinsame Darbietung dieses Stimulus mit einem aversiven Reiz in ihrer Erinnerung zu verzerren, d.h. sie wird die Assoziationshäufigkeit überschätzen. Dieses Phänomen soll in der vorliegenden Untersuchung genutzt werden. Wenn Rückenschmerzpatienten bestimmte Bewegungen als gefährlich ansehen, so sollten sie, wenn sie mit solchen Bewegungen konfrontiert werden und gleichzeitig ein negativer Reiz dargeboten wird, einen erhöhten Covariation Bias zeigen.

Wenn Fear-Avoidance Beliefs einem überdauernden Konzept über die Gefährlichkeit von Bewegungen entsprechen, dann sollten Patienten mit stärkeren Fear-Avoidance Beliefs bei der Konfrontation mit rückenschmerzrelevanten Bewegungen einen besonders deutlichen Covariation Bias zeigen.

2.3.2 Untersuchung der Konzepte Bewegungsangst und Fear-Avoidance Beliefs unter Verwendung von Fragebögen

Zur Überprüfung des Konzepts der Fear-Avoidance Beliefs soll der Fear-Avoidance Beliefs Questionnaire (FABQ) von Waddell et al. (1993) eingesetzt werden. Wie bereits weiter oben ausgeführt ist dies ein Fragebogen, der in einer Reihe von Untersuchungen von hoher Relevanz für die Vorhersage bestimmter Zielkriterien war. Tatsächlich stellten sich die individuellen Fragebogenscores als bester Prädiktor für die Rückkehr an den Arbeitsplatz heraus (26\% der Varianz wurde von den Fragebogenwerten aufgeklärt, nur $5 \%$ durch Aspekte wie Schmerzstärke, -dauer oder vorhandene Schädigung, Waddell et al. 1993). Der Aufklärungswert des Fragebogen für subjektive Beeinträchtigung der Patienten ist noch etwas höher und liegt bei über $30 \%$.

Der Fragebogen enthält 16 Items über subjektive Annahmen in Bezug auf den Zusammenhang zwischen Rückenschmerzen und Aktivitäten bzw. Arbeit. Aufgrund faktorenanalytischer Untersuchungen des Fragebogens geben Waddell et al. (1993) an, dass der Fragebogen zwei Bereiche erfasst: Fear-Avoidance Beliefs in Bezug auf die Arbeit und Fear-Avoidance Beliefs in Bezug auf körperliche Aktivität allgemein. Bei der 
Untersuchung der deutschen Version des FABQ fanden Pfingsten et al (2000) stattdessen eine Drei-Faktoren-Lösung. Dabei wurde der Faktor „Arbeit“ der englischen Version noch einmal aufgespalten: ein Faktor ("Ursache Arbeit") bezieht sich auf die Annahme der Patienten, dass die Arbeit eine Ursache für ihre Schmerzen sei. Der zweite Faktor ("Prognose") beschreibt die Angaben der Patienten über die Wahrscheinlichkeit, dass sie wieder in den Beruf zurückkehren. Der dritte Faktor entspricht dem in der englischen Version gefundenen Faktor "körperliche Aktivität". Bei einer multiplen Regression mit Beeinträchtigung (gemessen mit dem Funktionsfragebogen Hannover, revidierte Fassung, Raspe und Kohlmann 1994) und Arbeitsunfähigkeit als Kriterien zeigten sich die Faktoren 2 und 3 als beste Prädiktoren. Soziodemographische Faktoren wie Alter und Geschlecht und Symptomvariablen (Schmerzintensität, Dauer der Erkrankung, depressive Symptome und allgemeine Ängstlichkeit) klärten praktisch keine Varianz auf, wobei nur Schmerzintensität überhaupt einen gewissen Vorhersagewert hatte. Bei einer Diskriminanzanalyse von Patienten, die trotz Rückenschmerzen arbeiteten, und anderen, die aktuell arbeitsunfähig waren, zeigten sich signifikant höhere Werte in allen drei Faktoren des FABQ bei den arbeitsunfähigen Patienten. Besonders ausgeprägt war der Unterschied beim Faktor 2 (Prognose in Bezug auf Arbeitsfähigkeit).

Dass die mit dem Fragebogen erfragten Fear-Avoidance Beliefs einen starken Zusammenhang zu Beeinträchtigungsmaßen, insbesondere zu Arbeitsunfähigkeit aufweisen, ließ sich also zeigen. Allerdings ist bislang noch nicht genauer untersucht, ob das mit dem Fragebogen erfasste Konzept eher einen Zusammenhang zu tatsächlich erlebter Angst aufweist oder zu überdauernden Überzeugungen, die möglicherweise relativ unabhängig von Angst sind. Für eine inhaltliche Validierung des Konzepts des Fragebogens, um genauere Kenntnis darüber zu erhalten, was der FABQ erfasst, werden deshalb zwei eigene Fragebögen erstellt und mit dem FABQ korreliert. Dabei soll der eine tatsächlich erlebte Angst im Zusammenhang mit Schmerz messen (Schmerz-AngstBogen) und der andere eher generelle Einstellungen zu Aktivitäten bzw. Bewegungen und Schmerzen (Schmerz-Einstellungs-Bogen).

Der Schmerz-Angst-Bogen (SAB) soll Zustandsangst im Zusammenhang mit Schmerzerleben erfassen. Bei faktorenanalytischen Untersuchungen von Fragebögen zur Zustandsangst zeigten sich zumeist zwei Faktoren, die bei der Erstellung des Fragebogens berücksichtigt wurden, die Komponenten „Worry“ und „Emotionality“ (Liebert und Morris, 1967). „Worry“ bezieht sich dabei auf den eher kognitiven Aspekt 
der Angst: Items, die diesen Aspekt erfassen, beschreiben den gedanklichen Umgang mit Angst, so wie Selbstzweifel und Grübeln, Konzentration auf die Angst oder Sorge um die Zukunft. "Emotionality“ erfasst eher den motivational-emotionalen Charakter von Angst. Dazugehörige Items drücken die Wahrnehmung physiologischer Reaktionen des Körpers aus, so wie Anspannung, Neigung zum Weinen oder Herzklopfen. Dabei ist es wichtig zu betonen, dass die Wahrnehmung und Äußerung solcher Symptome nicht notwendigerweise in engem Zusammenhang mit tatsächlichen physiologischen Symptomen steht (Morris und Liebert, 1970).

Ein Fragebogen, der aktuelle Zustandsangst im Zusammenhang mit Schmerzerleben erfasst, sollte also Items enthalten, die diese beiden Bereiche abbilden. Alle Items des SAB sind vorgegebene Weiterführungen des Satzanfanges: „Wenn ich Schmerzen habe...“. Diese Weiterführungen beziehen sich alle auf aktuelles Erleben (z.B. „,..fühle ich mich beunruhigt.“). Damit wird angezielt, dass die Probanden wirklich Erfahrungen schildern, die unmittelbar im Zusammenhang mit ihren Schmerzen auftreten und nicht generelle Einstellungen und Meinungen. Die Items sind so formuliert, dass sie die beiden oben beschriebenen Bereiche „Worry“ und „Emotionality“ im Zusammenhang mit der Erfahrung von Schmerz abbilden (Beispielitems s. Abb. 2.4).

Als Anhaltspunkte für die Formulierung der Items galten die Items der Subskalen "Somatic Anxiety" und "Cognitive Anxiety" der Pain Anxiety Symptom Scale (McCracken, Zayfert und Gross, 1992), die ebenfalls Zustandsangst im Zusammenhang mit Schmerzerleben erfragt.

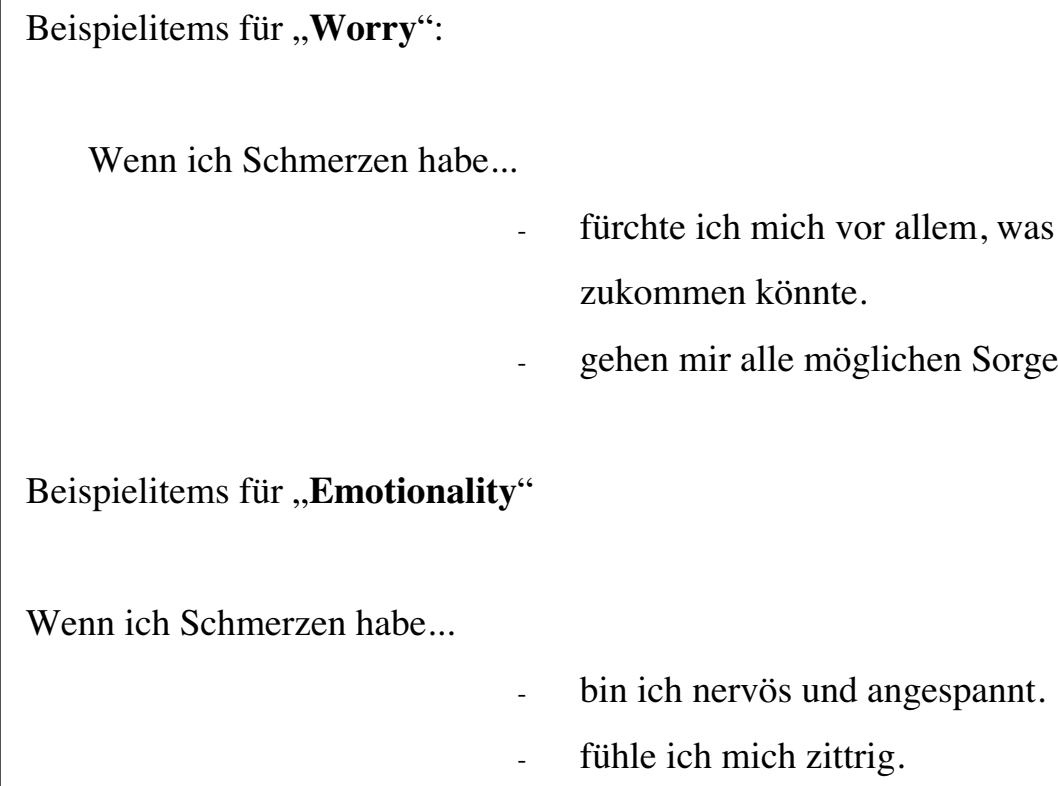

Abbildung 2.4 Beispielitems des SAB 
Der Schmerz-Einstellungs-Bogen (SEB) soll im Gegensatz zum SAB allgemeinere Einstellungen zum Zusammenhang zwischen Schmerz und Aktivitäten abbilden. Während der Schmerz-Angst-Bogen erfasst, welche Reaktionen Menschen auf tatsächlich erlebte Schmerzen zeigen, fragt der Schmerz-Einstellungs-Bogen, welches Verhalten beim Auftreten von Schmerzen von den Befragten generell für richtig erachtet wird. Einstellungen können sich auch ohne konkretes Erleben herausbilden, etwa durch Übernahme von Einstellungen wichtiger anderer Personen des sozialen Umfeldes. Bei dem hier untersuchten Zusammenhang von Bewegungen bzw. Aktivitäten und Schmerzen können solche Einstellungen z.B. von der Familie übernommen werden, aber auch von Ärzten oder aus den Medien. Einstellungen können Verhalten bestimmen, also z.B. Vermeidung hervorrufen, ohne dass die Person sich auf tatsächlich eigenes Erleben bezieht, das diese Vermeidung begründet. Das bedeutet, Menschen können z.B. bestimmte Objekte als gefährlich vermeiden, ohne dass sie selbst jemals deren Gefährlichkeit erlebt haben (Schiefele 1990). Im Zusammenhang mit Bewegungsvermeidung würde das also bedeuten, dass Menschen eventuell Bewegungen vermeiden, ohne dass sie sie selbst jemals als schmerzhaft erlebt haben.

Bei der Auswahl der Items für den Schmerz-Einstellungs-Bogen werden im Gegensatz zum Schmerz-Angst-Bogen allgemeine Formulierungen gewählt, wie „Menschen, die Schmerzen haben...“ (Beispielitems s. Abb. 2.5).

- Wer Schmerzen hat, sollte sich schonen.

- $\quad$ Ein Mensch, der Schmerzen hat, sollte sich möglichst wenig bewegen.

Abbildung 2.5 Beispielitems für SEB

Aufgrund der Tatsache, dass insbesondere der Faktor 1 des Fear-Avoidance Beliefs Questionnaires über arbeitsbezogene Beliefs einen guten Prädiktor für Beeinträchtigungsmaße darstellt, diskutieren Waddell et al. (1993) die Frage, ob nicht gerade der Faktor 1 anstatt Überzeugungen über die Schädlichkeit von Arbeit im Grunde Arbeitszufriedenheit erfasst. Allerdings untersuchen sie diesen Zusammenhang in ihrer Studie nicht explizit. In der vorliegenden Untersuchung soll deshalb die Arbeitszufriedenheit mit erhoben werden, um sie mit den Ergebnissen des FABQ in 
Beziehung setzen zu können. Als Instrument wird dafür der Arbeits-BeschreibungsBogen (ABB) von Neuberger und Allerbeck eingesetzt (1978).

Bei der Erfassung der Arbeitszufriedenheit erscheint es wichtig, verschiedene Bereiche des Arbeitslebens abzufragen, da die generelle Zufriedenheit am Arbeitsplatz von der Zusammenschau ganz unterschiedlicher Aspekte abhängt, z.B. der Art der Arbeit, der sozialen Beziehungen und der Entlohnung (Büssing, 1991). Der ABB wird dem gerecht, indem er neun verschiedene Aspekte von Arbeitszufriedenheit abfragt und zusätzlich ein Gesamturteil über die generelle Zufriedenheit abgeben lässt.

\subsection{Fragestellung und psychologische Hypothesen}

In der vorliegenden Untersuchung wird von der Annahme ausgegangen, dass sowohl Angst vor Bewegungen als auch sogenannte „Fear-Avoidance Beliefs“ Faktoren sind, die zur Beeinträchtigung und somit letztendlich zur Chronifizierung von Rückenschmerzen beitragen. Diese beiden Konzepte sollen im Experiment näher untersucht werden und ein möglicher Zusammenhang zwischen beiden Konzepten überprüft werden.

Zur Untersuchung der Bewegungsangst sollen das Startle-Paradigma und das Paradigma des Covariation Bias verwendet werden. Dabei gibt die Höhe des Startle-Reflexes Aufschluß über die emotional/motivationale Reaktion auf einen Stimulus, während der Covariation Bias Hinweise auf das Existieren kognitiver Schemata über die Gefährlichkeit eines bestimmten Stimulus gibt. Wenn Rückenschmerzpatienten im Alltag tatsächlich Angst vor Bewegungen haben, dann sollte diese Angst im Experiment durch die Vorstellung, bestimmte Bewegungen auszuführen, hervorzurufen sein. Dazu sollen Patienten mit chronischen Rückenschmerzen Bilder betrachten, die Menschen darstellen, die bestimmte Bewegungen ausführen. Damit die Patienten sich auch wirklich in die Bewegungssituation hineinversetzen, soll zusätzlich die Instruktion gegeben werden, sich vorzustellen, selbst diese Bewegung durchzuführen. Dabei ist darauf zu achten, dass die Bewegungen, die dargestellt werden, tatsächlich rückenschmerzrelevant sind, also Bewegungen darstellen, die von Rückenschmerzpatienten als potentiell schmerzauslösend erlebt werden. Typischerweise sind dies Drehbewegungen, Bücken oder Heben.

Sollte die Betrachtung bzw. die Vorstellung von rückenschmerzrelevanten Bewegungen tatsächlich Angst auslösen, so sollte sich dies sowohl bei der Startle-Reaktion als auch 
beim Covariation Bias zeigen. Das bedeutet, dass Patienten mit chronischem Rückenschmerz bei der Konfrontation mit Bildern von rückenschmerzrelevanten Bewegungen im Vergleich mit Bildern von angenehmen Bewegungen sowohl eine Erhöhung des Startle-Reflexes zeigen sollten als auch einen erhöhten Covariation Bias.

Wenn es einen Zusammenhang zwischen Fear-Avoidance Beliefs und Angst vor Bewegungen gibt, dann sollten insbesondere solche Patienten eine deutlichere Erhöhung der Startle-Reaktion bzw. des Covariation Bias aufweisen, die auf dem Fear-Avoidance Beliefs Questionnaire von Waddell et al. (1993) hohe Werte haben, die also in hohem Maße von einem Zusammenhang zwischen bestimmten Aktivitäten bzw. ihrer Arbeit und ihren Schmerzen überzeugt sind.

Um zu überprüfen, ob der Fear-Avoidance Beliefs Questionnaire eher emotional/motivationale Aspekte von Bewegungsangst erfasst oder eher von Bewegungsangst relativ unabhängige kognitive Schemata, wird der Zusammenhang des FABQ mit zwei selbst erstellten Fragebögen erhoben. Dabei erfasst der eine Fragebogen das tatsächliche Erleben von Angst vor Schmerzen (SAB), während der andere eher allgemeine Einstellungen zum Zusammenhang von Schmerz und Aktivitäten erfragt (SEB). Da Waddell et al. (1993) einen möglichen Zusammenhang zwischen FearAvoidance Beliefs und Arbeitszufriedenheit diskutieren, wird dieser Zusammenhang mit dem Arbeitsbeschreibungsbogen (ABB) von Neuberger und Allerbeck (1978) untersucht. Um einen möglichen Einfluss von Zustands- bzw. Eigenschaftsangst auf die Ergebnisse der experimentellen Phase kontrollieren zu können, wird zusätzlich das State-TraitAnxiety Inventory (STAI), deutsche Version von Laux, Glanzmann, Schaffner und Spielberger (1981) eingesetzt. Darüber hinaus werden die Zusammenhänge des FABQ, des SAB und des SEB mit den beiden Skalen des STAI erfasst, um zu überprüfen, ob die Konzepte dieser Fragebögen Verbindungen zu aktueller Zustandsangst bzw. zu genereller Ängstlichkeit aufweisen.

Die Validität der Startle-Untersuchung sowie der Erhebung des Covariation Bias soll überprüft werden, indem zusätzlich eine bereits gut untersuchte Methode zur Emotionsinduktion eingesetzt wird. Sowohl für die Startle-Reaktionsmessung als auch für die Messung des Covariation Bias werden neben den Bildern mit Bewegungen positive und negative Bilder des International Affective Picture Systems (IAPS) gezeigt. Diese Bilder wurden bereits mehrfach im Zusammenhang mit der Messung der Startle- 
Reaktion und des Covariation Bias eingesetzt, wobei sich in der Regel reliable Befundstrukturen über die Reaktion auf diese Bilder ergaben.

Sowohl die Bewegungsbilder als auch die Bilder der IAPS werden von allen Versuchspersonen in ihrer subjektiven Valenz und ihrem Arousal eingestuft. Dies dient dazu zu überprüfen, ob die Emotionsinduktion als gelungen gelten kann und ob die Bilder in der Erregungsdimension so stark eingeschätzt werden, dass überhaupt eine Veränderung der Startle-Reaktion erwartet werden kann (siehe Punkt 2.3.1). Zudem erlaubt dies die Prüfung der Hypothese, dass sich die Rückenschmerzpatienten von den Kontrollgruppen in ihrer Einschätzung der rückenschmerzrelevanten Bilder unterscheiden, d.h. dass sie diese negativer einschätzen.

Da die Konzepte der Bewegungsangst und der Fear-Avoidance Beliefs als spezifisch für Patienten mit Rückenschmerzen diskutiert werden, sollten auch die erwarteten Reaktionen bei der Startle-Ableitung und der Messung des Covariation Bias nur von Rückenschmerzpatienten (im Folgenden als CLBP-Patienten bezeichnet $=$ Chronic Low Back Pain) gezeigt werden. Um dies zu überprüfen, wird eine Kontrollgruppe gewählt, deren Personen ebenfalls unter chronischen Schmerzen leiden, für die aber Angst vor Bewegungen bzw. Fear-Avoidance Beliefs als nicht bedeutsam angesehen werden. Als eine solche Kontrollgruppe werden Patienten mit chronischen Kopfschmerzen untersucht. Bei chronischen Kopfschmerzen scheint Angst vor bestimmten Bewegungen keine Rolle zu spielen, da die Schmerzen unabhängig von Bewegungen auftreten. Bei Spannungskopfschmerzen ist es sogar häufig so, dass diese durch Bewegungen gelindert werden. Insofern ist davon auszugehen, dass Personen mit chronischen Kopfschmerzen keine Angst vor Bewegungen erleben und Bewegungen nicht aus Angst vor Schmerzen vermeiden. Darüber hinaus wird noch eine gesunde Kontrollgruppe untersucht, um eventuell generell schmerzspezifische Reaktionen von gesunden Reaktionen abgrenzen zu können. 
Folgende Hypothesen werden geprüft:

Hypothese 1:

Die Bewertung der Valenz der positiven und der negativen Bewegungsbilder unterscheidet sich bei allen drei Gruppen signifikant, wobei die CLBP-Patienten die Bilder von rückenschmerzrelevanten Bewegungen negativer beurteilen als die Kopfschmerzpatienten und die gesunde Kontrollgruppe.

Hypothese 2:

Der oben beschriebene Effekt ist besonders ausgeprägt bei Patienten, die auf dem Fear-Avoidance Beliefs Questionnaire hohe Scores zeigen.

Hypothese 3:

CLBP-Patienten zeigen eine deutliche Erhöhung des Startle-Reflexes bei der Betrachtung der Bilder mit rückenschmerzrelevanten Bewegungen im Vergleich zu Bildern von angenehmen Bewegungen, wohingegen Kopfschmerzpatienten und gesunde Kontrollpersonen diesen Effekt nicht zeigen.

Hypothese 4:

Der oben beschriebene Effekt ist besonders ausgeprägt bei Patienten, die auf dem Fear-Avoidance Beliefs Questionnaire hohe Scores zeigen.

Hypothese 5:

CLBP-Patienten zeigen einen stärkeren Covariation Bias in Bezug auf die rückenschmerzrelevanten Bilder als die Kopfschmerzpatienten und die andere Kontrollgruppe.

Hypothese 6:

Der oben beschriebene Effekt ist besonders ausgeprägt bei Patienten, die auf dem Fear-Avoidance Beliefs Questionnaire hohe Scores zeigen. 
Hypothese 7:

In Bezug auf die Bilder des IAPS ergeben sich die aus der Literatur bekannten Effekte in der Reaktion auf die positiven und negativen Bildern (Erhöhung der Startle-Reaktion und des Covariation Bias bei den negativen Bildern gegenüber der Reaktion auf die positiven Bilder). Es ergeben sich keine Unterschiede in der Beurteilung der IAPS-Bilder, der Höhe des Startle-Reflexes und im Covariation Bias zwischen den drei Gruppen.

Um eventuelle weitere Einflussfaktoren auf die Ergebnisse in Bezug auf die oben beschriebenen Hypothesen kontrollieren zu können, sollen folgende Fragen ebenfalls geprüft werden:

- Unterscheidet sich die State-Angst (gemessen mit dem State-Fragebogen des STAI) zwischen den Gruppen und wirkt sich die State-Angst auf die Höhe der Startle-Reaktion bei den Bewegungsbildern aus?

- Unterscheidet sich die Höhe der Arousal-Einschätzungen der Bewegungsbilder zwischen den Gruppen und gibt es Unterschiede in der Höhe der ArousalEinschätzung zwischen den Bewegungsbildern und den Bildern der IAPS?

- Gibt es Unterschiede bei der Einschätzung der Valenz der Bilder, der StartleReaktion und dem Covariation Bias abhängig davon, ob die Probanden zuerst die Bewegungsbilder gesehen haben (Block 1) oder zuerst die Bilder der IAPS (Block 2)? 
Des weiteren wird explorativ folgenden Fragestellungen nachgegangen:

1. Welche Zusammenhänge ergeben sich zwischen dem Fear Avoidance Beliefs Questionnaire und dem Schmerz-Angst-Bogen, dem Schmerz-Einstellungs-Bogen, dem Arbeits-Beschreibungs-Bogen und den beiden Skalen des State-Trait-Anxiety Inventory?

Weist der Fear-Avoidance Beliefs Questionnaire einen engeren Zusammenhang zum Schmerz-Angst-Bogen auf, d.h. wird mit ihm eher die erlebte Angst bei Schmerzen erfasst oder sind die Zusammenhänge zum Schmerz-Einstellungs-Bogen höher, d.h. der FABQ erhebt eher allgemeine Einstellungen zu Rückzugs- und Schonverhalten bei Schmerzen?

2. Welche Zusammenhänge ergeben sich zwischen dem Schmerz-Angst-Bogen und dem Schmerz-Einstellungsbogen untereinander, bzw. zwischen diesen beiden Bögen und dem Arbeits-Beschreibungs-Bogen und den beiden Skalen des State-TraitAnxiety Inventory?

3. Zeigen die CLBP-Patienten höhere Angst-Werte auf dem Schmerz-Angst-Bogen und stärkere Tendenzen zu Schonung und Vermeidung auf dem SchmerzEinstellungsbogen als die Personen der beiden Kontrollgruppen? 


\section{Methodik}

\subsection{Versuchsplan und unabhängige Variablen}

Der Untersuchung der Haupthypothesen liegt jeweils ein 3 x 2 faktorieller Versuchsplan mit den Faktoren Gruppe (Rückenschmerzpatienten, Kopfschmerzpatienten, gesunde Probanden) und Valenz der visuellen Stimuli (;) positiv/ :) negativ) mit Messwiederholung (s. Kapitel 4.2) zugrunde (s. Tabelle 3.1). Die jeweiligen Bildmaterialien (Bewegungsbilder / Bilder der IAPS) werden getrennt analysiert. Untersucht werden 36 Personen mit chronischen Rückenschmerzen, 18 Personen mit chronischen Kopfschmerzen und 18 gesunde Personen. Allen drei Gruppen werden jeweils 24 Bilder von Bewegungen und 24 Bilder des IAPS präsentiert (jeweils 12 (;) positive und 12 : negative Bilder). Zur Kontrolle von Reihenfolge- und Habituationseffekten werden die Bilder jeder Kategorie in sechs verschiedenen Bilderreihenfolgen dargeboten. Die Versuchspersonen der jeweiligen Gruppe werden den verschiedenen Reihenfolgen randomisiert zugewiesen. Grundsätzlich wird angestrebt, die Daten der beiden Kontrollgruppen zusammenzufassen. Sollte sich bei der Analyser der Daten allerdings zeigen, dass es Unterschiede auch zwischen den beiden Kontrollgruppen gibt, werden diese weiterhin getrennt betrachtet.

Tabelle 3.1: Versuchsplan

\begin{tabular}{|c|c|c|c|}
\hline \multirow{2}{*}{ Gruppen } & \multirow{2}{*}{$\begin{array}{l}\text { Experimentalgruppe } \\
\text { CLBP }(n=36)\end{array}$} & \multicolumn{2}{|l|}{ Kontrollgruppen } \\
\hline & & $\mathrm{KG} \mathrm{KS}(\mathrm{n}=18)$ & $\operatorname{KG~GES~}(\mathrm{n}=18)$ \\
\hline \multirow{4}{*}{ Stimuli } & $\begin{array}{l}12 \text { Bewegung }: ; \\
4 \text { mit Startle-Reiz }\end{array}$ & $\begin{array}{l}12 \text { Bewegung }(;) \\
4 \text { mit Startle-Reiz }\end{array}$ & $\begin{array}{l}12 \text { Bewegung }: ; \\
4 \text { mit Startle-Reiz }\end{array}$ \\
\hline & $\begin{array}{l}12 \text { Bewegung : } \\
4 \text { mit Startle-Reiz }\end{array}$ & $\begin{array}{l}12 \text { Bewegung : } \\
4 \text { mit Startle-Reiz }\end{array}$ & $\begin{array}{l}12 \text { Bewegung : } \\
4 \text { mit Startle-Reiz }\end{array}$ \\
\hline & $\begin{array}{l}12 \text { IAPS } \odot ; \\
4 \text { mit Startle-Reiz }\end{array}$ & $\begin{array}{l}12 \text { IAPS }: ; \\
4 \text { mit Startle-Reiz }\end{array}$ & $\begin{array}{l}12 \text { IAPS } \odot \\
4 \text { mit Startle-Reiz }\end{array}$ \\
\hline & $\begin{array}{l}12 \text { IAPS }: \\
4 \text { mit Startle-Reiz }\end{array}$ & $\begin{array}{l}12 \text { IAPS }: \\
4 \text { mit Startle-Reiz }\end{array}$ & $\begin{array}{l}12 \text { IAPS }: \\
4 \text { mit Startle-Reiz }\end{array}$ \\
\hline
\end{tabular}

CLBP = Rückenschmerzpatienten KG KS = Kopfschmerzpatienten KG GES = gesunde Probanden
Bewegung / IAPS $\odot$ : = positive Bilder Bewegung $/$ IAPS $:=$ negative Bilder 
Als unabhängige Variablen gelten die jeweilige Gruppenzugehörigkeit (CLBP, KG KS, KG GES) und die Valenz (:; / ()) der Bilder von Bewegungen bzw. der Bilder der IAPS.

\section{- Das Bildmaterial}

Zur Erstellung der Bewegungsbilder wurden insgesamt 44 Photos hergestellt. 22 Bilder zeigen angenehme Bewegungen (entspannt liegen oder gehen) und 22 unangenehme, rückenschmerzrelevante Bewegungen (bücken, heben). 18 Versuchspersonen mit chronischen Rückenschmerzen (Patienten der Schmerzambulanz Göttingen) wurden gebeten, die Bilder auf den Dimensionen Valenz und Arousal einzustufen. Für die experimentelle Untersuchung wurden jeweils die 12 positiven Bilder ausgewählt, die die höchsten Valenz- und die höchsten Arousal-Ratings aufweisen, sowie die 12 negativen Bilder, bei denen die Valenz-Ratings am niedrigsten und die Arousal-Ratings am höchsten sind (siehe Tabelle 3.1, Beispielbilder siehe Abbildungen 3.1 und 3.2). Auf den Bildern sind sowohl Frauen als auch Männer zu sehen, das Alter der dargestellten Personen variiert, um die Identifikationsmöglichkeiten mit den dargestellten Personen möglichst offen zu halten .

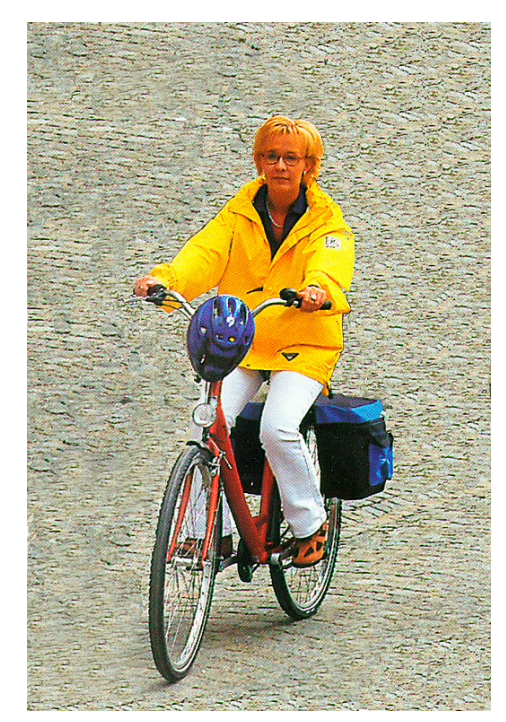

Abbildung 3.1 Beispiel Bewegung (:)

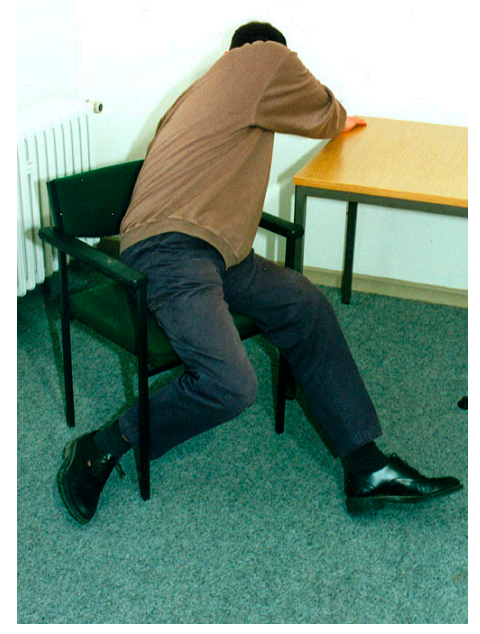

Abbildung 3.2 Beispiel Bewegung :: 
Tabelle 3.1 Bewegungsbilder, Mittelwerte und Standardabweichungen ( ) der Voreinstufung

\begin{tabular}{|l|l|l|}
\hline & Valenz & Arousal \\
\hline Positive Bilder $:-$ & $6.98(0.82)$ & $6.1(1,1)$ \\
\hline Negative Bilder $:-$ & $3.4(0.71)$ & $6.3(0.9)$ \\
\hline
\end{tabular}

Valenz: $1=$ sehr negativ, $9=$ sehr positiv

Arousal: $1=$ wenig erregend, $9=$ stark erregend

Tabelle 3.2 Kurzbeschreibung der Inhalte der Bewegungsbilder

\begin{tabular}{|c|c|}
\hline Inhalt & Valenz positiv/negativ \\
\hline Sitzen auf Sitzball & ;:) \\
\hline Liegen in Stufenlage & ;:) \\
\hline Stehen an Baum gelehnt & (:) \\
\hline Entspannt sitzen und lesen & ;: \\
\hline Entspannt sitzen und telefonieren & ;: \\
\hline Auf dem Boden sitzend lesen & ;:) \\
\hline Spaziergang & (:) \\
\hline Entspannt am Tisch sitzen, telefonieren & ;) \\
\hline An die Wand gelehnt lesen & (:) \\
\hline Bequem ausgestreckt stehen & ;) \\
\hline Liegen im Entspannungssessel & (;) \\
\hline Aufrecht Fahrrad fahren & ;) \\
\hline Drehbewegung auf Stuhl & :) \\
\hline Drehbewegung zum Telefon & : \\
\hline Vorbeugen zum Boden & : \\
\hline Koffer tragen & : \\
\hline Unter den Tisch beugen & : \\
\hline Kiste heben & : \\
\hline Drehbewegung mit Ball & : \\
\hline Über Fahrrad beugen & : \\
\hline Drehbewegung im Stehen & : \\
\hline Schnürsenkel zubinden gebeugt & : \\
\hline Beugebewegung zum unteren Schrankfach & : \\
\hline Unkrautjäten gebeugt & : \\
\hline
\end{tabular}


Aus dem 600 Bilder umfassenden Satz der IAPS werden 24 Bilder ausgewählt, jeweils 12 mit positiver und 12 mit negativer Valenz. Die Auswahl orientiert sich an Wertetabellen von Lang et al. (1997). Diese geben für jedes einzelne Bild gemittelte Bewertungen einer größeren Stichprobe von College-Studenten (Männer und Frauen) an, die alle Bilder auf den Dimensionen Valenz und Arousal auf einer neunstufigen Skala eingeschätzt haben (Kriterien für die Auswahl siehe Tabelle 3.3). Bei der Wahl der positiven Bilder werden Bilder mit sportlichen Inhalten (Skifahren, Schwimmen) ausgeschlossen, um keine Assoziation mit potentiell rückenschmerzrelevanten Bewegungen bei den CLBP Patienten hervorzurufen (Identifikationsnummern und Inhalte der ausgewählten Bilder siehe Tabelle 3.4).

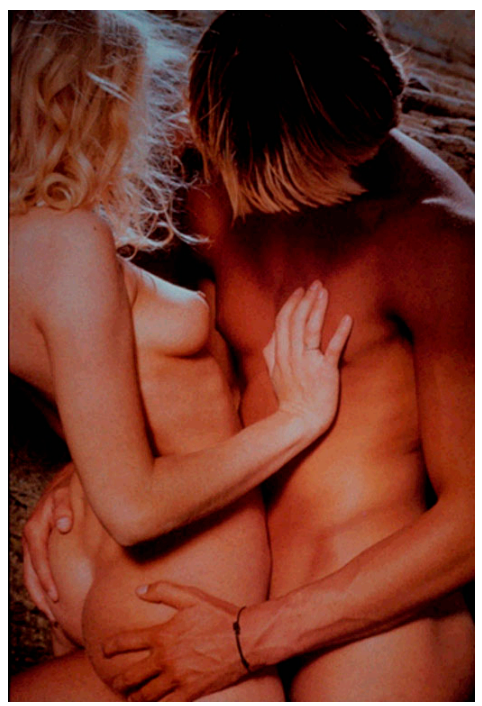

Abbildung 3.3 Beispiel IAPS :)

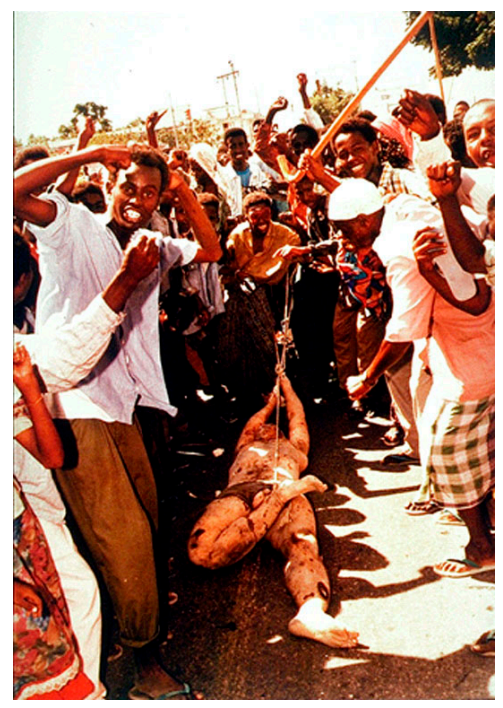

Abbildung 3.4 Beispiel IAPS :: 
Tabelle 3.3 Auswahlkriterien für IAPS Bilder (Einstufung nach Lang et al. 1997, Werte von Männern und Frauen zusammen)

\begin{tabular}{|l|l|l|}
\hline & Valenz & Arousal \\
\hline Positive Bilder $\odot ;$ & $>6.5$ & $>6$ \\
\hline Negative Bilder $\odot:$ & $<3$ & $>6$ \\
\hline
\end{tabular}

Valenz: $1=$ sehr negativ, $9=$ sehr positiv

Arousal: 1 = wenig erregend, $9=$ stark erregend

Tabelle 3.4 Identifikationsnummern und Kurzbeschreibung der Inhalte der IAPS Bilder

\begin{tabular}{|c|c|c|}
\hline Identifikationsnummer & Inhalt & Valenz positiv/negativ \\
\hline 1710 & Junge Hund & (:) \\
\hline 4607 & Pärchen Picknick & (;) \\
\hline 4608 & Pärchen halbnackt & (:) \\
\hline 4658 & Pärchen auf Bett & (;) \\
\hline 4659 & Pärchen nackt & (;) \\
\hline 4660 & Kuss & (;) \\
\hline 4670 & Pärchen stehend & (;) \\
\hline 4810 & Pärchen im Garten & (:) \\
\hline 5270 & Niagarafälle & (:) \\
\hline 5910 & Feuerwerk & ;) \\
\hline 8170 & Segelboot & (:) \\
\hline 8490 & Achterbahn & ;) \\
\hline 3010 & Mafiaopfer & (:) \\
\hline 3030 & Gesicht mit Narbe & : \\
\hline 3071 & Messerwunde am Hals & : \\
\hline 3530 & Überfall U-Bahn & (:) \\
\hline 6230 & Revolvermündung & (:) \\
\hline 6313 & Überfall & : \\
\hline 6350 & Bedrohung mit Messer & (:) \\
\hline 6540 & Überfall/ Würgegriff & : \\
\hline 9050 & Flugzeugabsturz & (:) \\
\hline 9252 & Lynchmord & : \\
\hline 9570 & Hundeskelett & (:) \\
\hline 9921 & Feuer & : \\
\hline
\end{tabular}




\subsection{Abhängige Variablen}

\subsubsection{Bildereinstufung}

Alle Bilder werden analog den Vorgaben von Lang et al. (1997) von den Probanden des Hauptversuchs nach dem Durchgang mit den Startle-Reizen auf einer 9- stufigen Skala auf der Dimension Valenz und Arousal eingestuft (s. Abbildung 3.5, Evaluationsbogen siehe Anhang B7).

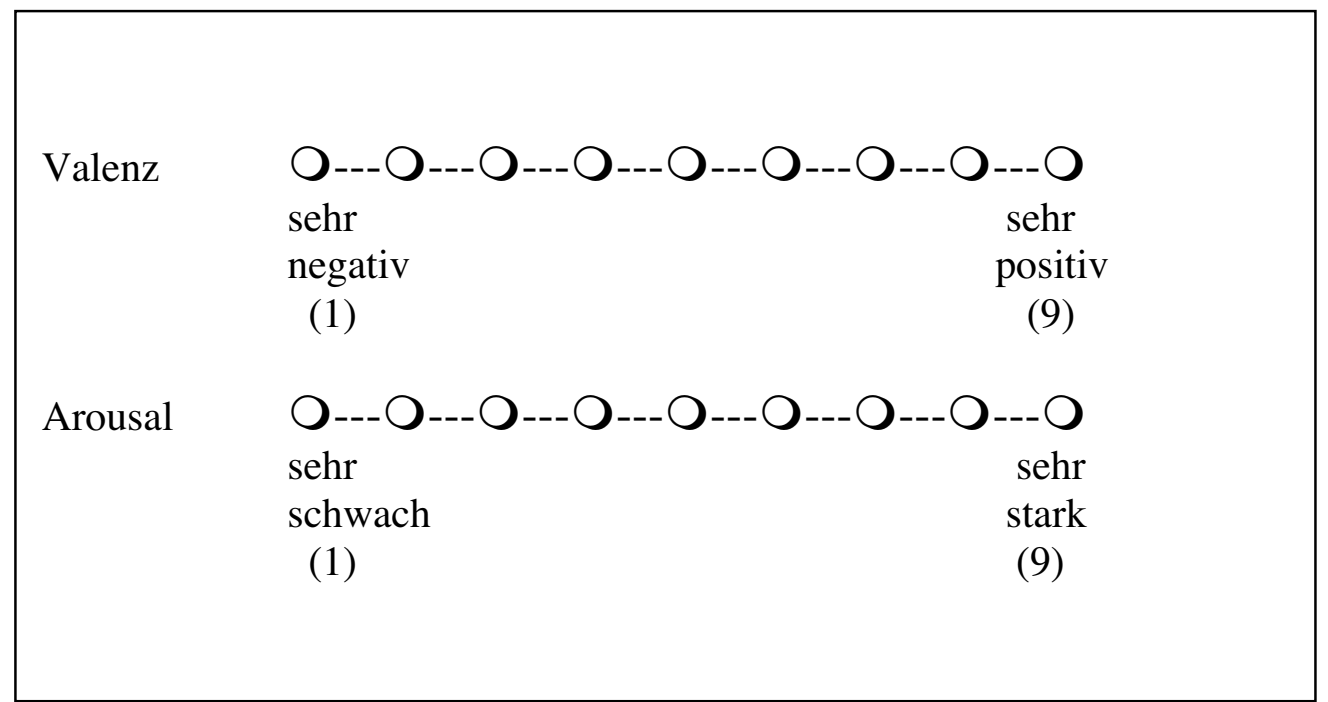

Abbildung 3.5 Skalen für die Bestimmung von Valenz- und Arousal

\subsubsection{Auslösung und Ableitung der Startle-Reaktion}

Als Startle-Reiz dient ein 50 Millisekunden dauerndes, 105 dB(A) lautes weißes Rauschen, das über Kopfhörer präsentiert wird. Der Startle-Reiz setzt unmittelbar ein (extrem steile Anstiegsflanke). Das digitalisierte weiße Rauschen wird über die AudioAusgabe eines Macinthosh Power PC (7600) abgespielt und mittels einer Hifiverstärkers (Denon) verstärkt. Die Startle-Reaktion wird über das EMG des Orbicularis Oculi, abgeleitet mit zwei Hellige Silber-Silberchlorid-Elektroden (Durchmesser $0.5 \mathrm{~cm}$ ) unter dem linken Auge, erhoben. Eine Erdungselektrode (Durchmesser $1 \mathrm{~cm}$ ) befindet sich hinter dem linken Ohr. Die Elektroden werden gefüllt mit Hellige-Elektrodengel (deutsche Patentnummer: 1564 103). Die analoge Aufbereitung und Verstärkung des 
EMG erfolgt mit einem Vitaport II mit 8-Kanal-Universal-Verstärker-Analogmodul. Die Güte der Ableitung wird online überprüft. Das Vitaport-Gerät digitalisiert das EMG mit 12 Bit Wandlungsbreite. Gemessen wird mit einer Sample-Frequenz von $2560 \mathrm{~Hz}$, mit einem Hochpass von $67 \mathrm{~Hz}$ und einem Tiefpass von $1000 \mathrm{~Hz}$. Das Rohwertesignal wird gleichgerichtet, integriert und geglättet entsprechend den Empfehlungen von Berg und Balaban (1999). Der gesamte Versuchsablauf inklusive Steuerung des Startle-Reizes, Konfiguration und Ansteuerung des Verstärkers, Anstoß der Messung, Auslesen der physiologischen Daten, erster digitale Aufbereitung sowie Abspeicherung erfolgt durch ein Steuerprogramm für den Macintosh von Breuer (1998). Als abhängige Variable dient das Maximum der geglätteten Kurve (Differenz zwischen Baseline und Maximum der Kurve) in einem Zeitfenster von 39 bis 120 Millisekunden nach dem Startle-Reiz. Als Baseline dient der Mittelwert der Kurve von 500ms vor dem StartleReiz bis zum Startle-Reiz.

Da nicht auszuschließen ist, dass die Reaktion auf die Bewegungsbilder bei einer bezogen auf den Gesamtpool randomisierten (gemischten) Darbietung durch die Reaktion auf die IAPS-Bilder in unkontrollierbarer Weise beeinflusst wird, werden die Bilder dieser beiden verschiedenen Quellen getrennt gezeigt. Jede Versuchsperson sieht zunächst alle 24 Bilder der einen Quelle und danach alle Bilder der anderen Quelle. Um den Einfluss der Reihenfolge kontrollieren zu können, werden der Hälfte der Versuchspersonen zu Beginn die Bewegungsbilder gezeigt, der anderen die Bilder der IAPS. Wenn ein Startle-Stimulus mit jedem Bild assoziiert würde, wäre eine starke Habituation der Reaktion zu erwarten (s. Vrana et al., 1988), deshalb wird bei jeder Bilderpräsentation (also der Darbietung aller Bilder pro Proband) nur bei einem Drittel der Bilder ein Startle-Reiz dargeboten, jeweils gleich häufig bei positiven und negativen Bildern. Von den 24 Bewegungsbildern und den 24 IAPS-Bildern werden also je 8 Bilder (4 positive und 4 negative Bilder) mit einem Schreckreiz assoziiert.

Da davon auszugehen ist, dass die Startle-Reaktion dennoch in gewissem Maße habituieren wird, wird jedes Bild zweimal, nämlich jeweils einmal in der ersten Hälfte einer Bilderpräsentation und einmal in der zweiten Hälfte, zusammen mit einem StartleReiz präsentiert. Damit werden Habituationseffekte für die einzelnen Kategorien ausgeglichen. Da bei jeder Bilderpräsentation pro Proband nur bei einem Drittel der Bilder ein Startle-Reiz gesetzt wird, benötigt man drei verschiedene Bilderreihenfolgen, um alle Bilder einmal zusammen mit einem Startle-Reiz zu zeigen. Da alle Bilder 
insgesamt zweimal mit dem Startle-Reiz präsentiert werden sollen, ergeben sich sechs verschiedene Bilderreihenfolgen.

Zur Erstellung der verschiedenen Bilderreihenfolgen werden zunächst die 12 Bilder jeder Kategorie (Bewegung $(-) /:$, IAPS $(-) /()$ ) in vier Zellen aufgeteilt. In jeder Zelle wird jeweils einem Bild der Startle-Reiz zugewiesen (Beispiel s. Tabelle 3.6, grau unterlegt). Die Bilder werden in den weiteren fünf Bilderreihenfolgen so verteilt, dass die oben beschriebene Anforderung (jedes Bild wird einmal in der ersten Hälfte der Präsentation und einmal in der zweiten Hälfte mit Startle-Reiz präsentiert) erfüllt ist. Darüber hinaus soll jedes Bild in jeder der vier Zellen einmal vertreten sein, so dass gewährleistet ist, dass jedes Bild an unterschiedlichen Positionen gezeigt wird und so Reihenfolgeeffekte der Bilderpräsentation ebenfalls kontrolliert sind. Für jede der vier Kategorien (Bewegung $: /(:)$, IAPS $:(:)$ ) wird eine Tabelle mit je vier Zellen in sechs verschiedenen Reihenfolgen erstellt (Beispiel s. Tabelle 3.6).

Tabelle 3.6 Aufteilung der 12 Bewegungsbilder :) auf die verschiedenen Zellen zur Erstellung der Bilderreihenfolgen: exemplarische Darstellung der Reihenfolgebildung

\begin{tabular}{|c|c|c|c|c|c|c|}
\hline & \begin{tabular}{|l|} 
B+ für \\
Reihenfolge 1
\end{tabular} & $\begin{array}{l}\text { B+ für } \\
\text { Reihenfolge } 2\end{array}$ & $\begin{array}{l}\text { B+ für } \\
\text { Reihenfolge } 3\end{array}$ & $\begin{array}{l}\text { B+ für } \\
\text { Reihenfolge } 4\end{array}$ & $\begin{array}{l}\text { B+ für } \\
\text { Reihenfolge } 5\end{array}$ & $\begin{array}{l}\text { B+ für } \\
\text { Reihenfolge } 6\end{array}$ \\
\hline Zelle 1 & $\begin{array}{l}\mathrm{B}+1 \\
\mathrm{~B}+2 \\
B+3\end{array}$ & $\begin{array}{l}\mathrm{B}+11 \\
\mathrm{~B}+4 \\
B+7 \\
\end{array}$ & $\begin{array}{l}B+5 \\
B+10 \\
B+9\end{array}$ & $\begin{array}{l}\mathrm{B}+6 \\
B+12 \\
\mathrm{~B}+8 \\
\end{array}$ & $\begin{array}{l}B+2 \\
B+9 \\
B+5\end{array}$ & $\begin{array}{l}\mathrm{B}+5 \\
\mathrm{~B}+9 \\
B+4\end{array}$ \\
\hline Zelle 2 & $\begin{array}{l}\mathrm{B}+4 \\
\mathrm{~B}+5 \\
B+6\end{array}$ & $\begin{array}{l}B+1 \\
B+10 \\
B+12\end{array}$ & $\begin{array}{l}\mathrm{B}+2 \\
\mathrm{~B}+8 \\
B+11\end{array}$ & $\begin{array}{l}\mathrm{B}+7 \\
B+9 \\
\mathrm{~B}+3\end{array}$ & $\begin{array}{l}B+8 \\
B+6 \\
B+3\end{array}$ & $\begin{array}{l}\mathrm{B}+11 \\
\mathrm{~B}+2 \\
\mathrm{~B}+10\end{array}$ \\
\hline Zelle 3 & $\begin{array}{l}B+7 \\
B+8 \\
B+9\end{array}$ & $\begin{array}{l}B+5 \\
B+3 \\
B+6\end{array}$ & $\begin{array}{l}B+1 \\
B+12 \\
B+4\end{array}$ & $\begin{array}{l}B+2 \\
B+11 \\
B+10\end{array}$ & $\begin{array}{l}\mathrm{B}+11 \\
\mathrm{~B}+7 \\
B+4\end{array}$ & $\begin{array}{l}\mathrm{B}+7 \\
B+3 \\
\mathrm{~B}+1\end{array}$ \\
\hline Zelle 4 & $\begin{array}{l}\mathrm{B}+10 \\
B+11 \\
\mathrm{~B}+12\end{array}$ & $\begin{array}{l}B+9 \\
B+8 \\
B+2\end{array}$ & $\begin{array}{l}\mathrm{B}+3 \\
\mathrm{~B}+7 \\
B+6\end{array}$ & $\begin{array}{l}\mathrm{B}+5 \\
\mathrm{~B}+4 \\
B+1\end{array}$ & $\begin{array}{l}\mathrm{B}+12 \\
B+10 \\
\mathrm{~B}+1\end{array}$ & $\begin{array}{l}B+8 \\
B+12 \\
B+6\end{array}$ \\
\hline
\end{tabular}

$\mathrm{B}+3$ : Bild Nr. 3 mit Startle-Reiz präsentiert

Um jeweils eine Bilderreihenfolge mit positiven und negativen Bildern zu erzeugen, werden die erstellten Tabellen für die positiven und die negativen Bewegungsbilder zusammengefügt, ebenso wie die Tabellen für die positiven und die negativen IAPSBilder. Diese zusammengefügten Präsentationspläne zeigen die Bilderreihenfolgen auf, wie sie den einzelnen Probanden präsentiert werden. Dabei wird gewährleistet, dass die Rangsummen der Positionen der jeweiligen Kategorien (:)/:) gleich sind, so dass die Positionen der Bilder der beiden Kategorien vergleichbar sind. Als zusätzliche 
Randbedingung gilt: es werden maximal zwei Bilder einer Kategorie hintereinander präsentiert (Beispiel s. Tabelle 3.7, alle Bilderreihenfolgen s. Anhang B5).

Die Bilder werden insgesamt 6 Sekunden lang gezeigt, der Startle-Reiz erfolgt 3, 4 oder 5 Sekunden nach dem Erscheinen des Bildes auf dem Monitor (s. Vrana 1988). Diese Variation soll sicherstellen, dass die Probanden keine festen Erwartungen darüber bilden können, wann der Startle-Reiz erfolgt und so die Schreckreaktion unter Umständen abgeschwächt wird. In jeder Bilderreihenfolge werden jeweils sechsmal Startle-Reize in den Pausen zwischen den Bildern präsentiert, um Daten über die Höhe des StartleReflexes ohne Emotionsinduktion durch Bilder zu erhalten. Zudem wird dadurch eine Erwartungsbildung über die Auftretenswahrscheinlichkeit eines Startle-Reizes zusätzlich erschwert. Die Pause zwischen den Bildern variiert zwischen 27 und 48 Sekunden.

Tabelle 3.7 Reihenfolge 1 (positive und negative Bilder)

\begin{tabular}{|c|c|c|}
\hline Bildernummer & Rangplatz (:) & Rangplatz : \\
\hline $1+$ & 1 & \\
\hline 14 & & 2 \\
\hline $21-$ & & 3 \\
\hline \multicolumn{3}{|l|}{ Blank 1} \\
\hline $2+$ & 4 & \\
\hline $3+$ & 5 & \\
\hline $17-$ & & 6 \\
\hline \multicolumn{3}{|l|}{ Blank 2} \\
\hline $4+$ & 7 & \\
\hline $20-$ & & 8 \\
\hline $18-$ & & 9 \\
\hline $5+$ & 10 & \\
\hline \multicolumn{3}{|l|}{ Blank 3} \\
\hline $15-$ & & 11 \\
\hline $6+$ & 12 & \\
\hline $23-$ & & 13 \\
\hline $7+$ & 14 & \\
\hline 19 & & 15 \\
\hline $8+$ & 16 & \\
\hline \multicolumn{3}{|l|}{ Blank 4} \\
\hline $9+$ & 17 & \\
\hline 16- & & 18 \\
\hline $10+$ & 19 & \\
\hline \multicolumn{3}{|l|}{ Blank 5} \\
\hline $24-$ & & 20 \\
\hline $11+$ & 21 & \\
\hline $22-$ & & 22 \\
\hline $13-$ & & 23 \\
\hline \multicolumn{3}{|l|}{ Blank 6} \\
\hline $12+$ & 24 & \\
\hline Rangsumme & 150 & 150 \\
\hline
\end{tabular}

22- : Bild Nr.22 (negativ) assoziiert mit Startle-Reiz

Blank 3: Startle-Reiz in Bildpause 
Von den 72 Versuchspersonen werden jeder Reihenfolge insgesamt 12 Versuchspersonen zugeordnet, wobei jeweils 6 Probanden zuerst die Bewegungsbilder sehen und 6 Probanden zuerst die IAPS-Bilder, so dass 12 Reihenfolgeblöcke entstehen. Die genaue Verteilung der Versuchspersonen auf diese Blöcke zeigt Tabelle 3.8. Da jede Kontrollgruppe von der Anzahl der Personen her die Hälfte der Personen der Experimentalgruppe aufweist, ergibt sich, dass jedem Block 3 Personen der Experimentalgruppe und jeweils 2 Personen der einen Kontrollgruppe und eine Person der anderen Kontrollgruppe zugeordnet wird. Wenn in Block 1 eine Person der Kopfschmerzgruppe ist, werden Block 2 zwei Personen der Kopfschmerzgruppe zugeteilt und umgekehrt. Über alle Bilderreihenfolgen sehen jeweils 18 Personen der Experimentalgruppe (d.h. die Hälfte der Experimentalgruppe) und je 9 Personen beider Kontrollgruppen (d.h. je die Hälfte der beiden Kontrollgruppen) die Bilder der einen oder der anderen Bildquelle (Bewegungsbilder/IAPS) zuerst. Unter Berücksichtigung dieser Vorgaben werden die Probanden den Reihenfolgen und Blöcken zufällig zugeteilt.

Da jedes Bild in zwei der sechs Reihenfolgen zusammen mit dem Startle-Reiz präsentiert wird und jede Reihenfolge von 12 Personen gesehen wird, gibt es von jedem Bild insgesamt 24 Einzeldaten über die Höhe des Startle-Reflexes.

Die Bilder werden präsentiert über einen 19-Zoll Monitor, vor dem die Probanden in einem Abstand von $75 \mathrm{~cm}$ sitzen.

Tabelle 3.8 Verteilung der Versuchspersonen auf die Reihenfolgeblöcke

\begin{tabular}{|c|c|c|c|c|c|c|c|}
\hline & & $\begin{array}{l}\text { Reihenfolge } \\
1\end{array}$ & $\begin{array}{l}\text { Reihenfolge } \\
2\end{array}$ & $\begin{array}{l}\text { Reihenfolge } \\
3\end{array}$ & $\begin{array}{l}\text { Reihenfolge } \\
4\end{array}$ & $\begin{array}{l}\text { Reihenfolge } \\
5\end{array}$ & $\begin{array}{l}\text { Reihenfolge } \\
6\end{array}$ \\
\hline $\begin{array}{l}\text { Block } \\
1\end{array}$ & $\begin{array}{l}\text { 1. Bewegung } \\
\text { 2. IAPS }\end{array}$ & $\begin{array}{l}3 \text { CLBP } \\
2 \text { KG KS } \\
1 \text { KG GES }\end{array}$ & $\begin{array}{l}3 \text { CLBP } \\
2 \text { KG KS } \\
1 \text { KG GES }\end{array}$ & $\begin{array}{l}3 \text { CLBP } \\
1 \mathrm{KG} \mathrm{KS} \\
2 \mathrm{KG} \mathrm{GES}\end{array}$ & $\begin{array}{l}3 \text { CLBP } \\
1 \text { KG KS } \\
2 \text { KG GES }\end{array}$ & $\begin{array}{l}3 \text { CLBP } \\
2 \mathrm{KG} \mathrm{KS} \\
1 \mathrm{KG} \mathrm{GES}\end{array}$ & $\begin{array}{l}3 \text { CLBP } \\
1 \mathrm{KG} \mathrm{KS} \\
2 \mathrm{KG} \mathrm{GES}\end{array}$ \\
\hline $\begin{array}{l}\text { Block } \\
2\end{array}$ & $\begin{array}{l}\text { 1.IAPS } \\
\text { 2. Bewegung }\end{array}$ & $\begin{array}{l}3 \text { CLBP } \\
1 \mathrm{KG} \mathrm{KS} \\
2 \mathrm{KG} \mathrm{GES}\end{array}$ & $\begin{array}{l}3 \text { CLBP } \\
1 \mathrm{KG} \mathrm{KS} \\
2 \mathrm{KG} \mathrm{GES}\end{array}$ & $\begin{array}{l}3 \text { CLBP } \\
2 \text { KG KS } \\
1 \text { KG GES }\end{array}$ & $\begin{array}{l}3 \text { CLBP } \\
2 \text { KG KS } \\
1 \text { KG GES }\end{array}$ & $\begin{array}{l}3 \text { CLBP } \\
1 \mathrm{KG} \mathrm{KS} \\
2 \mathrm{KG} \mathrm{GES}\end{array}$ & $\begin{array}{l}3 \text { CLBP } \\
2 \text { KG KS } \\
1 \text { KG GES }\end{array}$ \\
\hline
\end{tabular}

CLBP = Rückenschmerzpatienten KG KS = Kopfschmerzpatienten KG GES = gesunde Probanden 


\subsubsection{Covariation Bias}

Zur Erhebung des Covariation Bias werden den Versuchspersonen die Bilder nach dem Durchgang des Startle-Experiments ein weiteres Mal gezeigt, wobei sie angeben sollen, ob das jeweilige Bild zusammen mit dem "lauten Ton" (Startle-Reiz) präsentiert worden ist (Instruktion siehe Anhang B7).

\subsubsection{Fragebögen}

Der Fear-Avoidance Beliefs Questionnaire von Waddell et al. (1993) in der deutschen Version von Pfingsten et al. (2000) dient der Erfassung von Überzeugungen über den Zusammenhang zwischen Rückenschmerzen und Arbeit bzw. körperliche Aktivität Die 16 Items werden auf einer sieben-stufigen Skala von "Stimmt gar nicht" bis "Stimmt genau“ beantwortet. Wie in Kapitel 2.3.2 beschrieben fanden Pfingsten et al. (2000) bei einer Validierungsuntersuchung der deutschen Version des Fragebogens statt einer ZweiFaktoren-Lösung eine eindeutige Drei-Faktoren-Lösung mit Eigenwerten größer als 1.5. Der erste Faktor "Ursache Arbeit" erklärt $43.4 \%$ der Varianz, der zweite Faktor "Prognose" 11.8 \% Varianz. Der dritte Faktor "körperliche Aktivität" erklärt 8.9\% der Varianz. Die Homogenitätswerte der einzelnen Faktoren sind mit über 0.80 durchgehend gut, ebenso wie die Werte für die Retest-Reliabilität aller drei Faktoren (ebenfalls $>0.80$ ). Da der FABQ explizite Aussagen über Rückenschmerzen macht, erhalten ihn nur die Probanden der Experimentalgruppe.

Alle Probanden die an dem Versuch teilnehmen, also auch die Probanden der beiden Kontrollgruppen, erhalten den Schmerz-Angst-Bogen und den Schmerz-EinstellungsBogen. Beide Fragebögen wurden für die vorliegende Studie entwickelt. Sie enthalten Aussagen über Schmerzen im allgemeinen und können deshalb von allen Probanden ausgefüllt werden. Die Auswahl der Items orientierte sich an den Empfehlungen von Mummendey (1995) zur Fragebogenkonstruktion. Die Antworten werden auf einer sechsstufigen Antwortskala gegeben, die von ,trifft genau zu“ bis „trifft überhaupt nicht

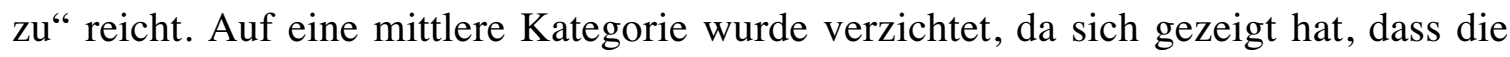
Interpretation einer mittleren Kategorie uneindeutig ist und von verschiedenen Probanden verschieden aufgefasst werden kann. Ansonsten sollten die Antworten klar 
verständlich sein, und von jedem Probanden generell auch erlebbar. Dennoch sollten keine trivialen Antworten enthalten sein, die von jedem als richtig beantwortet würden (z.B. „Wenn ich Schmerzen habe, fühle ich mich nicht sehr wohl.“).

Um die Reliabiltät des SAB zu untersuchen, wurde der Fragebogen 28 Studierenden der Universität Göttingen zum Ausfüllen vorgelegt. Alle Items wurden gut verstanden. Als Maß für die interne Konsistenz des Fragebogen wurde Cronbach's Alpha verwendet. Ein Item erwies sich als die interne Konsistenz des Fragebogens verringernd und wurde deshalb herausgenommen. Der Fragebogen mit 15 Items hatte eine gute interne Konsistenz von 0.86. Zwei Wochen nach der ersten Bearbeitung füllten 21 Studenten den Fragebogen ein weiteres Mal aus. Die Retest-Reliabilität von $r=0.82$ ist ebenfalls als gut zu bezeichnen.

Für den SEB wurden zunächst 13 Items formuliert, die allgemeine Aussagen über das Verhalten, das Menschen mit Schmerzen zeigen sollten, enthalten (z.B. „Ein Mensch, der Schmerzen hat, sollte sich schonen."). An einer Stichprobe von 60 Studierenden der Universität Göttingen wurde der Fragebogen voruntersucht. Zwei Items wurden herausgenommen, da sie die interne Konsistenz des Fragebogens verringerten. Mit 11 Items zeigte der Fragebogen eine gute interne Konsistenz von 0.83. Nach zwei Wochen wurde der Fragebogen 20 Personen ein weiteres Mal vorgelegt. Es ergab sich ein RetestReliabiltätskoeffizient von $\mathrm{r}=0.71$, also geringer als der Wert des Schmerz-AngstBogens, aber immer noch als zufrieden stellend zu bewerten.

Zur weiteren Überprüfung des Konzepts der Fear-Avoidance Beliefs wird den Probanden eine gekürzte Version des Arbeitsbeschreibungsbogens von Neuberger und Allerbeck (1978) vorgelegt. Der Arbeitsbeschreibungsbogen erfragt die Zufriedenheit in neun verschiedenen Bereichen des Arbeitslebens (Kollegen, Vorgesetze, Tätigkeit, Arbeitsbedingungen, Organisation und Leitung, eigene Entwicklung, Bezahlung, Arbeitszeit und Sicherheit des Arbeitsplatzes), sowie die Zufriedenheit mit der Arbeitsstelle im Ganzen. Die Antwortmöglichkeiten reichen auf einer sieben-stufigen Skala von „sehr zufrieden“ bis „sehr unzufrieden“. Die Originalversion enthält für alle neun Unterbereiche jeweils konkrete Unterfragen zusätzlich zur Frage, wie zufrieden man insgesamt mit diesem Bereich ist. Da die Korrelationen zwischen den einzelnen Fragen für die Unterbereiche und den jeweiligen Gesamtfragen alle bei über 0.70 liegen (Neuberger und Allerbeck, 1978), wird bei der vorliegenden Studie auf die Einzelfragen 
verzichtet und nur die Frage zur allgemeinen Zufriedenheit für den jeweiligen Bereich gestellt. Die Retest-Reliabilität des Fragebogens (nach vier Wochen) wird von den Autoren mit 0.73 angegeben und kann als zufrieden stellend angesehen werden.

Um die Auswirkung allgemeiner Zustandsangst auf die Untersuchung kontrollieren zu können, wird zu Beginn der Untersuchung das State-Trait-Angstinventar (STAI), deutsche Version von Laux, Glanzmann, Schaffner und Spielberger (1981) eingesetzt. Die State-Skala des STAI dient zur Erfassung der aktuellen Zustandsangst der Probanden. Sie besteht aus 20 Items wobei 10 negativ formuliert sind und 10 positiv, das heißt 10 Items spiegeln einen Zustand der Angst wider und 10 einen Zustand der Ruhe bzw. Entspannung. Die Items sind Aussagen über den momentanen Zustand (z.B. „Ich bin ruhig.“), die Antwortalternativen gehen vier-stufig von ,überhaupt nicht“ bis „sehr“. Die interne Konsistenz kann als hoch bezeichnet werden (Cronbachs $\alpha \geq 0.90$ ), die Retest-Reliabilität liegt bei Testwiederholung nach einer Stunde bei 0.74.

Da die Startle-Reaktion unter anderem auch von genereller Ängstlichkeit abhängig ist (Cook et al. 1991), soll überprüft werden, ob sich die Personen der drei untersuchten Gruppen auf diesem Faktor unterscheiden. Dazu wird die Trait-Skala des STAI eingesetzt. Die Trait-Skala erfasst Angst als überdauernde Eigenschaft und bezieht sich auf die generelle Neigung, in vielen verschiedenen Situationen mit Angst zu reagieren. Auch sie besteht aus 20 Items, wobei hier 13 Items in Richtung von Ängstlichkeit formuliert sind und 7 Items in Richtung von Gelassenheit. Die Items sind als generelle Aussagen formuliert (z.B. „Ich bin vergnügt.“), Antworten werden auf einer vier-stufigen Skala gegeben, die von „fast nie“ bis „fast immer“ geht. Die interne Konsistenz der Skala ist nach Angaben der Autoren ebenfalls hoch (Cronbachs $\alpha \geq 0.90$ ), die RetestReliabilität nach 63 Tagen liegt bei 0.84 .

\subsection{Probanden}

36 Patienten mit chronischen Rückenschmerzen nehmen an dem Versuch teil. Alle Patienten befinden sich zum Zeitpunkt der Untersuchung in Behandlung in der Schmerzambulanz der Universität Göttingen. Während einer Voruntersuchung für die Teilnahme an einem speziellen Rehabilitationsprogramm werden sie über die Studie informiert und um eine Teilnahme gebeten. Bei allen teilnehmenden Patienten ist die 
Diagnose „unspezifischer Rückenschmerz“ gestellt worden. Ausschlusskriterien für die Untersuchung sind schwere Atem- und Herz-/Kreislauferkrankungen, spezifische Rückenschmerzen aufgrund von Tumoren oder Entzündungen, akuter Bandscheibenvorfall und Zustand nach Bauch- oder gynäkologischen Operationen. Drei Patienten sind nicht werktätig, sondern Hausfrauen bzw. Rentner. 19 der Patienten sind aktuell arbeitsunfähig aufgrund ihrer Rückenschmerzen (Arbeitsfehlzeit im Mittel 16.5 Wochen).

Als Schmerzkontrollgruppe werden 18 Patienten mit chronischen Kopfschmerzen (Migräne und Spannungskopfschmerzen) weit gehend über Zeitungsanzeigen rekrutiert. Voraussetzung für die Teilnahme ist, dass die Patienten seit mehr als drei Jahren an Kopfschmerzen leiden, sich deswegen in ärztlicher Behandlung befinden und keine zusätzlichen Rückenschmerzprobleme aufweisen. Als gesunde Kontrollgruppe nehmen 18 Personen ohne Schmerzproblematik an der Untersuchung teil. Vorwiegend sind dies Angestellte der Universität. Bei der Auswahl der Probanden wird angestrebt, beide Kontrollgruppen nach Alter und Geschlecht mit der CLBP-Gruppe zu parallelisieren.

\subsection{Versuchsdurchführung}

\subsubsection{Räumliche Bedingungen}

Die Untersuchung findet in den Räumen des Georg-Elias-Müller Instituts statt. Der Untersuchungsraum ist ungefähr 30 qm groß und ist mit einer 8 qm großen, elektrisch abgeschirmten Versuchskabine ausgestattet. Der Laborcomputer zur Registrierung der Daten steht außerhalb der Versuchskabine und ist durch die Probanden nicht einzusehen. In der Kabine befindet sich ein Tisch mit einem 19 Zoll-Monitor. In ungefähr $75 \mathrm{~cm}$ Abstand davon steht ein bequemer Stuhl. Das Vitaportgerät zur Ableitung des EMG befindet sich in der Kabine mit einer Verbindung zum Laborcomputer. An beiden Seiten des Tisches sind Abschirmungen angebracht, so dass die Versuchspersonen während des Versuchs nicht im Raum umherschauen können. 
3.4.2 Versuchsablauf

Tabelle 3.10: Versuchsablauf

Informed Consent

Fragebogen: FABQ (nur CLBP)

STAI

Anbringen der Elektroden/ Kopfhörer

Überprüfung der Ableitung

Instruktion

Startle-Experiment:

Bilderreihenfolge $1-6$, Block 1 bzw. 2

Ableitung des EMG vom Orbicularis Oculi

Bewertung der Bilder (Valenz, Arousal):

Bilderreihenfolge 1-6 (wie vorher, ohne Startle)

Covariation Bias:

Einschätzung der Assoziationshäufigkeit von

Startle-Reiz und Bild

Startle-Experiment:

Bilderreihenfolge $1-6$, Block 1 bzw. 2

Ableitung des EMG vom Orbicularis Oculi

Bewertung der Bilder (Valenz, Arousal):

Bilderreihenfolge 1-6 (wie vorher, ohne Startle)

Covariation Bias:

Einschätzung der Assoziationshäufigkeit von

Startle-Reiz und Bild

Fragebogen: SAB

SEB

$\mathrm{ABB}$ 
Der geplante Versuchsablauf wird vor Beginn der Untersuchung der Ethik-Kommision der Deutschen Gesellschaft für Psychologie vorgelegt und von dieser genehmigt. Die Probanden erhalten die Information, dass bei dieser Studie die körperliche Reaktion von Schmerzpatienten auf bestimmte Bilder gemessen werde. Nachdem sie schriftlich darüber aufgeklärt worden sind, dass sie verschiedene Bilder zu sehen bekommen, ihr Lidschlagreflex registriert wird und sie von Zeit zu Zeit einen lauten Ton über Kopfhörer hören, unterzeichnen sie eine Einverständniserklärung (siehe Anhang A2) und füllen den STAI aus, die CLBP Patienten auch den FABQ. Sie werden dann in die Versuchskabine geführt. Dort werden die Messelektroden unter dem linken Auge angebracht, sowie eine Erdungselektrode hinter dem linken Ohr. Um die korrekte Ableitung des Lidschlagreflexes zu gewährleisten, wird ein willkürliches Zwinkern der Probanden online am Bildschirm des Laborcomputers beobachtet. Die Probanden werden instruiert, während der gesamten Bilderpräsentation auf den Bildschirm zu schauen. Vor der Präsentation der Bewegungsbilder werden die Probanden aufgefordert, sich während der Betrachtung der Bilder vorzustellen, dass sie selbst die Bewegung ausführen. In den Pausen zwischen den Bildern erscheint auf dem Bildschirm ein Kreuz, auf das die Probanden schauen sollen, um zu gewährleisten, dass der Blick die ganze Zeit auf den Bildschirm gerichtet bleibt. Nach der Präsentation der Bilder mit Applizierung des Startle-Reizes wird die Bilderserie den Probanden noch einmal präsentiert, wobei sie die Bilder in ihrer Valenz und ihrem Arousal einstufen sollen. Weiter werden sie befragt, ob das entsprechende Bild zusammen mit dem lauten Ton dargeboten wurde (Covariation Bias). Bei diesem Versuchsabschnitt können sich die Probanden die Bilder so lange anschauen, wie sie wollen und dann selbst mit der Maus das nächste Bild anwählen. Danach wird die noch nicht gezeigte Bilderserie präsentiert, wieder zunächst ein Durchgang mit Applizierung des Startle-Reizes und danach ein Durchgang ohne StartleReiz, zur Erhebung des Covariation Bias und der Evaluation der Bilder. Nach der Präsentation der Bilder werden die Patienten außerhalb der Versuchskabine gebeten, den SAB, den SEB und wenn sie erwerbstätig sind, auch den ABB auszufüllen. Abschließend werden genauere Informationen über die Untersuchung und die Fragestellung gegeben und eventuell noch verbleibende Fragen geklärt. 


\section{Ergebnisse}

\subsection{Eingangsmerkmale der Stichprobe}

An der Untersuchung nehmen 36 CLBP-Patienten und jeweils 18 Probanden der beiden Kontrollgruppen (KG KS und KG GES) teil. Es werden ungefähr gleich viele Männer wie Frauen untersucht, wobei die Zahl der Männer etwas überwiegt. Im Schnitt sind die Probanden zwischen 43 und 45 Jahren alt. Die CLBP-Patienten leiden im Mittel seit dreieinhalb Jahren an ihren Schmerzen, die Kopfschmerzpatienten im Mittel seit 21 Jahren. Insgesamt sind mehr Probanden mit geringeren Bildungsabschlüssen (mittlere Reife/ Hauptschule) vertreten.

Bei der Auswahl der Teilnehmer der beiden Kontrollgruppen wurde eine Parallelisierung in Bezug auf Alter, Geschlecht, Bildungsstand und Ängstlichkeit angestrebt. Ob diese Parallelisierung gelungen ist, wird statistisch überprüft; für die Variable Alter und Ängstlichkeit (gemessen mit der Trait-Skala des STAI) mittels einer einfaktoriellen ANOVA für unabhängige Stichproben, für die Variablen Geschlecht und Bildungsstand mittels eines Chi-Quadrat-Tests. Es ergeben sich auf keiner der drei Variablen signifikante Unterschiede, so dass davon ausgegangen werden kann, dass die untersuchten Gruppen in diesen Bereichen vergleichbar sind (Ergebnisse siehe Tabelle 4.2). Eine Parellelisierung der beiden Schmerzgruppen in Bezug auf die Dauer der Schmerzen war insofern nicht möglich, da bei den Kopfschmerzpatienten der Symptombeginn meist sehr viel früher liegt, was dazu führt, dass sie bereits eine wesentlich längere Zeit an ihren Schmerzen leiden als die Rückenschmerzpatienten.

Für die Untersuchung der Startle-Reaktion ist es notwendig, dass die Probanden auf die Präsentation des Reizes (lauter Ton) überhaupt eine deutliche Veränderung des EMGs am Augenmuskel zeigen. Insgesamt 13 Personen zeigten keine Reaktion und werden deshalb aus der Analyse für die Hypothesen drei und vier herausgenommen. Die Frage, ob sich diese Non-Responder signifikant unterschiedlich auf die drei Gruppen verteilen, wird ebenfalls mit Hilfe eines Chi-Quadrat-Tests überprüft. Wie die Ergebnisse in Tabelle 4.2 zeigen, ergeben sich auch hier keine signifikanten Unterschiede.

Um zu überprüfen, ob die CLBP-Patienten in Bezug auf die Schmerzstärke und ihre Beeinträchtigung mit anderen in Behandlung befindlichen Patienten mit chronischen Rückenschmerzen vergleichbar sind, wird ihnen eine 11-Punkte numerischen RatingSkala zur Einschätzung ihrer Schmerzstärke vorgelegt. Die Beeinträchtigung wird über 
den Funktionsfragebogen Hannover (FFbH) von Raspe und Kohlmann (1994) erfasst. Die Werte unterscheiden sich im Mittel nicht von denen einer Stichprobe mit 190 Patienten aus der Schmerzambulanz der Universität Göttingen (siehe Anhang C1).

Tabelle 4.1 Stichprobencharakteristika

\begin{tabular}{|c|c|c|c|}
\hline & \multirow{2}{*}{\begin{tabular}{|l} 
Experimental- \\
gruppe
\end{tabular}} & \multicolumn{2}{|c|}{ Kontrollgruppen } \\
\hline & & KG KS & KG GES \\
\hline \multirow{2}{*}{$\begin{array}{l}\text { Männlich } \\
\text { Weiblich }\end{array}$} & 19 & 9 & 11 \\
\hline & 17 & 9 & 7 \\
\hline Alter: M (SD) & $45(10)$ & $43(12)$ & $45(9)$ \\
\hline $\begin{array}{l}\text { Schmerzdauer in Jahren: } \\
\mathrm{M}(\mathrm{SD})\end{array}$ & $3.5(3,5)$ & $21(12)$ & --- \\
\hline \multirow{2}{*}{$\begin{array}{l}\text { Abitur } \\
\text { Hauptschule/mittlere Reife }\end{array}$} & 4 & 6 & 4 \\
\hline & 32 & 12 & 14 \\
\hline $\begin{array}{l}\text { Trait-Skala des STAI: } \\
\text { M (SD) }\end{array}$ & $2.18(0.63)$ & $2.31(0.61)$ & $1.89(0.52)$ \\
\hline $\begin{array}{l}\text { Responder / } \\
\text { Non-Responder }\end{array}$ & $27 / 9$ & $17 / 1$ & $15 / 3$ \\
\hline $\begin{array}{l}\text { Beeinträchtigung }(\mathrm{FFbH}) \text { : } \\
\mathrm{M} \text { (SD) }\end{array}$ & $61.9(19.4)$ & --- & --- \\
\hline $\begin{array}{l}\text { Schmerzstärke } \\
\text { (11-Punkte VAS) : M (SD) }\end{array}$ & $6.3(2.1)$ & --- & --- \\
\hline
\end{tabular}

CLBP $=$ Rückenschmerzpatienten

KG KS = Kopfschmerzpatienten

KG GES = gesunde Probanden 
Tabelle 4.2 Stichprobencharakteristika: Ergebnisse des Gruppenvergleichs

\begin{tabular}{||l|c|c|c|}
\hline & \multicolumn{2}{|l|}{ Gruppenvergleich } & $\mathrm{p}=.77$ \\
\hline Geschlecht & $\mathrm{df}=2$ & $\chi^{2}=.51$ & $\mathrm{p}=.77$ \\
\hline Alter & $\mathrm{df}=2$ & $\mathrm{~F}=.26$ & $\mathrm{p}=.14$ \\
\hline Bildungsgrad & $\mathrm{df}=2$ & $\chi^{2}=3.9$ & $\mathrm{p}=.10$ \\
\hline $\begin{array}{l}\text { Trait anxiety } \\
\text { Verteilung }\end{array}$ & $\mathrm{df}=2$ & $\mathrm{~F}=2.35$ & $\mathrm{p}=.21$ \\
\hline $\begin{array}{l}\text { Non- } \\
\text { Responder }\end{array}$ & $\mathrm{df}=2$ & $\chi^{2}=3.1$ & \\
\hline
\end{tabular}

\section{2 Überprüfung der Hypothesen}

Die Hypothesen werden mit verschiedenen inferenz-statistischen Verfahren geprüft, die jeweils bei den Ergebnissen der einzelnen Hypothesen vorgestellt werden. Für die Feststellung einer statistischen Signifikanz wird bei allen Hypothesen ein $\alpha$ von 0.05 erwartet.

\section{Hypothese 1}

In Bezug auf die Bewegungsbilder wird angenommen, dass es einen signifikanten Unterschied in der Bewertung der positiven und der negativen Bilder gibt und dass die CLBP-Patienten die rückenschmerzrelevanten Bilder negativer einschätzen als die beiden Kontrollgruppen, sich jedoch in der Einschätzung der positiven Bilder nicht von diesen unterscheiden. Für die Prüfung dieser Hypothese wird eine 3 x 2 ANOVA “Gruppe“(CLBP, KG KS, KG GES) x "Qualität" (;)/:) gerechnet. Da die Stichprobe in Bezug auf den Faktor "Qualität" abhängig ist (die Bilder werden jeweils von der gleichen Person eingeschätzt) handelt es sich um eine ANOVA mit Messwiederholung. 
Bei der Wahl eines parametrischen statistischen Verfahrens wird Intervallskalierung, Normalverteilung der Grundgesamtheit und Homogenität der Fehlervarianzen vorausgesetzt. Auch wenn das Skalenniveau von Rating-Skalen nicht eindeutig ist, kann nach der Literatur ein Intervallskalenniveau angenommen werden (Bortz und Döring, 1995). Auf die explizite Überprüfung der Voraussetzungen der Normalverteilung bzw. der Homogenität der Fehlervarianzen wird verzichtet, was insofern vertretbar ist, da nach Bortz, Lienert und Boehnke (1990) die Verletzung der Voraussetzungen die Bestätigung einer Unterschiedshypothese eher erschwert, also insgesamt eine konservative Testung bedeutet.

Bei der Berechnung der ANOVA wird ein signifikanter Haupteffekt "Qualität" erwartet, sowie ein signifikanter Interaktionseffekt. Tatsächlich zeigen die Ergebnisse einen sehr signifikanten Haupteffekt "Qualität“" ( $<$ < 0.001) und einen Interaktionseffekt ( $<<0.01$, s. Tabelle 4.4). Die positiven und die negativen Bewegungsbilder werden also von allen drei Gruppen signifikant unterschiedlich wahrgenommen. Abbildung 4.1 zeigt, dass die CLBP-Patienten die rückenschmerzrelevanten Bilder wie erwartet negativer bewerten als die beiden anderen Gruppen.

Tabelle 4.3 Einschätzung der Valenz der Bilder:

Mittelwerte und Standardabweichungen ( )

\begin{tabular}{|l|l|l|l|}
\hline & CLBP & KG KS & KG GES \\
\hline Bewegung $:-$ & $7.08(0.74)$ & $6.81(0.79)$ & $7.03(0.81)$ \\
\hline Bewegung $:$ & $3.24(1.19)$ & $4.29(0.96)$ & $3.9(1.21)$ \\
\hline
\end{tabular}

Tabelle 4.4 Valenz der Bewegungsbilder: Ergebnisse der Varianzanalyse

\begin{tabular}{|l|l|l|l|}
\hline & $\mathrm{F}$ & $\mathrm{df}$ & $\mathrm{p}$ \\
\hline Gruppe (CLBP, KG KS, KG GES) & 2.41 & $2 / 69$ & 0.97 \\
\hline Qualität (:) / : ) & 341.43 & $1 / 69$ & $<0.0001^{* * *}$ \\
\hline Gruppe x Qualität & 5.73 & $2 / 69$ & $0.005^{* *}$ \\
\hline *** $\leq 0.001 \quad * * \mathrm{p} \leq 0.01$ &
\end{tabular}




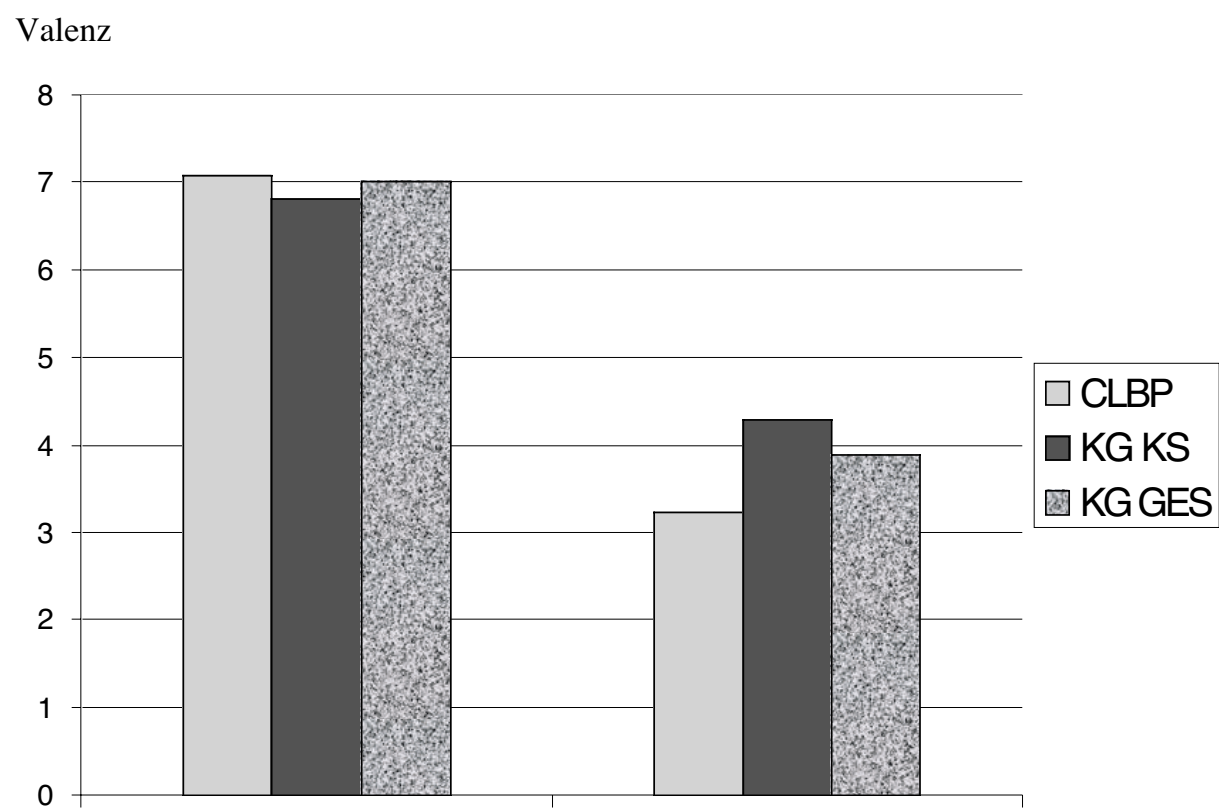

Bilder

Bewegung $;$

Bewegung $:$

Abbildung 4.1: Einschätzung der Valenz der Bilder der drei Gruppen

\section{Hypothese 2}

Es wird davon ausgegangen, dass die negative Einschätzung der rückenschmerzrelevanten Bewegungsbilder bei den CLBP-Patienten, die auf dem FABQ hohe Scores zeigen, besonders ausgeprägt ist. Die Einteilung der Probanden der CLBPGruppe in die Subgruppen Fear-avoidance hoch/niedrig erfolgt anhand des Medians des Summenscores des FABQ. 18 Patienten mit einem Summenscore über bzw. gleich 64 werden als Gruppe mit hoher Fear-avoidance, 18 Patienten mit einem Summenscore unter 64 als Gruppe mit geringer Fear-avoidance eingestuft (Mittelwert und Standardabweichung der Summenscores siehe Tabelle 4.5).

Tabelle 4.5 Mittelwerte und Standardabweichungen der Summenscores des FABQ

\begin{tabular}{|l|l|l|l|}
\hline Subgruppe & Anzahl & Mittelwert & Standardabweichung \\
\hline Fear-Avoidance hoch $(\geq 64)$ & 18 & 75.5 & 7.7 \\
\hline Fear-Avoidance niedrig $(<64)$ & 18 & 35.9 & 17.3 \\
\hline
\end{tabular}


Um zu überprüfen, ob die Patienten mit hohen Scores auf dem FABQ die rückenschmerzrelevanten Bewegungsbilder tatsächlich negativer einstufen als die mit weniger ausgeprägten Beliefs, wird nur für die CLBP-Patienten eine ANOVA mit Messwiederholung "Subgruppe“ (Fear-Avoidance hoch/niedrig) x "Qualität" (Bewegung (:) / :) ) gerechnet. Erwartet wird ein Haupteffekt "Qualität" und ein Interaktionseffekt. Den Haupteffekt "Qualität" zeigt das Ergebnis der Varianzanalyse. Es ergibt sich kein Haupteffekt für die Subgruppe und auch nicht der angenommene Interaktionseffekt. Die Personen mit ausgeprägteren Fear-Avoidance Beliefs bewerten die rückenschmerzrelevanten Bilder nicht negativer als die mit weniger ausgeprägten Beliefs (Ergebnisse s. Tabellen 4.6 und 4.7).

Tabelle 4.6 Valenz der Bewegungsbilder in Abhängigkeit von Fear-Avoidance: Mittelwerte und Standardabweichungen ( )

\begin{tabular}{|l|l|l|}
\hline Subgruppe & Fear-Avoidance hoch & Fear-Avoidance niedrig \\
\hline Bewegung $: ;$ & $7.13(0.74)$ & $7.02(0.76)$ \\
\hline Bewegung $\odot ;$ & $3.38(1.25)$ & $3.09(1.13)$ \\
\hline
\end{tabular}

Tabelle 4.7 Valenz der Bewegungsbilder in Abhängigkeit von Fear-Avoidance: Ergebnisse der Varianzanalyse

\begin{tabular}{|l|l|l|l|}
\hline & $\mathrm{F}$ & $\mathrm{df}$ & $\mathrm{p}$ \\
\hline Subgruppe (FA hoch/ niedrig) & 0.96 & $1 / 34$ & 0.33 \\
\hline Qualität (:) / :) & 205.42 & $1 / 34$ & $<0.0001 * * *$ \\
\hline Subgruppe x Qualität & 0.13 & $1 / 34$ & 0.72 \\
\hline
\end{tabular}

$* * * \mathrm{p} \leq 0.001$

\section{Hypothese 3}

Es wird erwartet, dass die CLBP-Patienten in Bezug auf die negativen Bewegungsbilder eine deutliche Erhöhung des Startle-Reflexes zeigen im Vergleich zu den positiven Bildern. Bei den Kopfschmerzpatienten und den gesunden Kontrollgruppen wird dieser Effekt nicht vorhergesagt.

Auch für die Überprüfung dieser Hypothese wird eine 3 x 2 ANOVA "Gruppe" (CLBP, KG KS, KG GES) x "Qualität" (;)/:) mit Messwiederholung gerechnet (Mittelwerte 
und Standardabweichungen der Startle-Reaktion auf die Bilder siehe Tabelle 4.8). Für die Messwerte der Startle-Reaktion (A/D units) kann Intervallskalenniveau angenommen werden. Auf die Überprüfung der anderen Voraussetzungen wird auch hier verzichtet. In die Berechnung der Varianzanalyse gehen Daten von 59 Personen ein, da wie weiter oben erwähnt, die 13 Non-Responder aus der Berechnung ausgeschlossen werden.

Tabelle 4.8 Startle-Reaktion auf Bewegungsbilder : Mittelwerte und Standardabweichungen (A/D units) (nur Responder)

\begin{tabular}{|l|l|l|l|}
\hline & CLBP & KG KS & KG GES \\
\hline Bewegung $:-$ & $376.8(314.7)$ & $396.67(377.85)$ & $295.1(199.4)$ \\
\hline Bewegung $:-$ & $397.84(348.3$ & $368.21(321.7)$ & $316.35(203.9)$ \\
\hline
\end{tabular}

In der Hypothese wird ein Interaktionseffekt angenommen, den die Ergebnisse der Varianzanalyse jedoch nicht zeigen. Die CLBP-Patienten zeigen also nicht, wie angenommen, eine Erhöhung der Startle-Reaktion bei der Betrachtung der rückenschmerzrelevanten Bilder (s. Tabelle 4.9).

Tabelle 4.9 Startle-Reaktion auf Bewegungsbilder (nur Responder): Ergebnisse der Varianzanalyse

\begin{tabular}{|l|l|l|l|}
\hline & F & df & $\mathrm{p}$ \\
\hline Gruppe (CLBP, KG KS, KG GES) & 0.38 & $2 / 56$ & 0.68 \\
\hline Qualität (:) / :) ) & 0.071 & $1 / 56$ & 0.79 \\
\hline Gruppe x Qualität & 0.9 & $2 / 56$ & 0.42 \\
\hline
\end{tabular}

In dieser Berechnung werden die jeweiligen Mittelwerte der Startle-Reaktion auf die verschiedenen Bilder betrachtet. Eine ebenso plausible Vorgehensweise ist es, pro Person einen individuellen Differenzwert zu berechnen (Bewegung $:$ minus Bewegung $(;)$ ) und mit einer einfaktoriellen Varianzanalyse zu überprüfen, ob es einen Gruppenunterschied für diesen Differenzwert gibt, d.h. ob die CLBP-Patienten einen höheren Differenzwert aufweisen als die beiden Kontrollgruppen. Auch diese Berechnungsvariante ergibt keine Effekte. Beide Berechnungsarten zeigen also gleichgerichtete Ergebnisse (siehe Anhang C2). 


\section{Hypothese 4}

Es wird angenommen, dass die in Hypothese 3 vorhergesagten Ergebnisse besonders deutlich ausgeprägt sind bei Personen, die auf dem FABQ hohe Scores aufweisen.

Um zu überprüfen, ob die Patienten der Subgruppe "Fear-Avoidance hoch" eine stärkere Startle-Reaktion auf die rückenschmerzrelevanten Bilder zeigen als die Patienten der Subgruppe "Fear-Avoidance niedrig" wird für die CLBP-Patienten eine ANOVA mit Messwiederholung "Subgruppe" (Fear-Avoidance hoch/niedrig) x "Qualität" (Bewegung (:) / :) ) gerechnet (Mittelwerte und Standardabweichung der Reaktion der beiden Subgruppen siehe Tabelle 4.10). Die Ergebnisse der Varianzanalyse zeigen, dass die beiden Gruppen nicht unterschiedlich reagieren, es zeigen sich keine Haupteffekte und auch nicht der erwartete Interaktionseffekt. Die Gruppe der Patienten mit ausgeprägten Fear-Avoidance Beliefs zeigt also keine stärkere Startle-Reaktion auf die rückenschmerzrelevanten Bilder als die anderen Patienten (s. Tabelle 4.11).

Tabelle 4.10 Startle-Reaktion auf Bewegungsbilder in Abhängigkeit von Fear-Avoidance: Mittelwerte und Standardabweichung ( ) (nur Responder)

\begin{tabular}{|l|l|l|}
\hline & Fear-Avoidance hoch $(\geq 64, \mathrm{n}=12)$ & Fear-Avoidance niedrig $(<64, \mathrm{n}=15)$ \\
\hline Bewegung $: ;$ & $321.96(247.23)$ & $420.73(362.28)$ \\
\hline Bewegung $: ;$ & $321.67(254.67)$ & $458.8(406.63)$ \\
\hline
\end{tabular}

Tabelle 4.11 Startle-Reaktion auf Bewegungsbilder in Abhängigkeit von Fear-Avoidance (nur Responder): Ergebnisse der Varianzanalyse

\begin{tabular}{|l|l|l|l|}
\hline & F & df & p \\
\hline Subgruppe (FA hoch/ niedrig) & 0.89 & $1 / 25$ & 0.36 \\
\hline Qualität (; / :) & 0.46 & $1 / 25$ & 0.51 \\
\hline Subgruppe x Qualität & 0.47 & $1 / 25$ & 0.5 \\
\hline
\end{tabular}




\section{Hypothese 5}

Auch für die Ergebnisse des Covariation Bias gilt die Annahme, dass die CLBPPatienten sich in der Reaktion auf die rückenschmerzrelevanten Bilder von den beiden Kontrollgruppen unterscheiden, indem sie häufiger die falsche Angabe machen, dass das Bild mit dem Startle-Reiz zusammen präsentiert wurde. Unterschiede in der Reaktion auf die positiven Bilder werden nicht vorhergesagt.

Um eventuelle Unterschiede zwischen den Gruppen feststellen zu können und insbesondere, um Auskunft über mögliche Interaktionseffekte zu erhalten, soll auch hier eine 3 x 2 ANOVA "Gruppe“ (CLBP, KG KS, KG GES) x "Qualität" (:) /:) mit Messwiederholung gerechnet werden. Dafür wird für jeden einzelnen Probanden ein Summenscore für die Anzahl der falsch positiven Antworten pro Qualität (= falsche Angabe, dass das Bild mit dem Startle-Reiz assoziiert war) berechnet. Da insgesamt 12 Bilder pro Kategorie gezeigt wurden und vier davon jeweils tatsächlich zusammen mit dem aversiven Reiz dargeboten wurden, sind maximal acht falsche Antworten möglich. Für den Summenscore kann Intervallskalenniveau angenommen werden. Auf die Überprüfung der anderen Voraussetzungen wird ebenso wie bei der Bewertung der Valenzen und der Berechnung der Startle-Reaktion verzichtet. Die Ergebnisse der Varianzanalyse zeigen keine Haupteffekte "Gruppe" und "Qualität" (Mittelwerte und Standardabweichungen für die falsch positiven Antworten der Probanden s. Tabelle 4.12, Ergebnisse der Varianzanalyse s. Tabelle 4.13). Es gibt jedoch, wie die Hypothese vorhersagt, einen Interaktionseffekt $(\mathrm{p}<0.01)$. Die Abbildung 4.2 zeigt, dass dieser Interaktionseffekt einerseits wie erwartet darauf zurückzuführen ist, dass die CLBPPatienten häufiger bei den rückenschmerzrelevanten Bildern die Angabe machen, dass dieses Bild zusammen mit dem Startle-Reiz präsentiert wurde. Andererseits treffen die Kopfschmerzpatienten diese falsche Aussage häufiger bei den positiven Bewegungsbildern, was nicht vorhergesagt wurde. 
Tabelle 4.12 Covariation Bias bei Bewegungsbildern: Mittelwerte und Standardabweichungen ( ) der Summenscores der falsch positiven Antworten

\begin{tabular}{|l|l|l|l|}
\hline & CLBP & KG KS & KG GES \\
\hline Bewegung $\odot:$ & $2.06(1.72)$ & $3.11(1.75)$ & $1.89(1.88)$ \\
\hline Bewegung $:$ & $3.14(2.55)$ & $1.89(1.32)$ & $2.44(2.06)$ \\
\hline
\end{tabular}

Tabelle 4.13 Covariation Bias bei Bewegungsbildern: Ergebnisse der Varianzanalyse

\begin{tabular}{|l|l|l|l|l|}
\hline & & $\mathrm{F}$ & $\mathrm{df}$ & $\mathrm{p}$ \\
\hline \multirow{3}{*}{ Bewegung } & Gruppe (CLBP, KG KS, KG GES) & 0.44 & $2 / 69$ & 0.65 \\
\cline { 2 - 5 } & Qualität (:) / : ) & 0.23 & $1 / 69$ & 0.64 \\
\cline { 2 - 5 } & Gruppe x Qualität & 5.83 & $2 / 69$ & $0.005 * *$ \\
\hline
\end{tabular}

falsch positive

Antworten

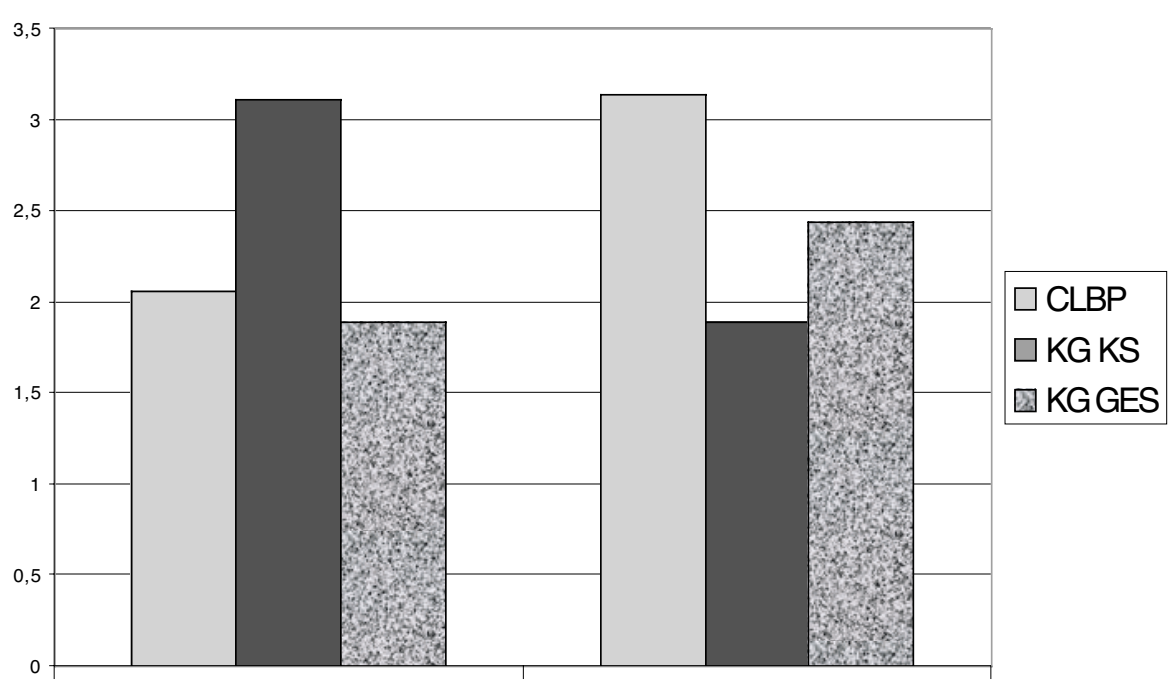

Bilder

Bewegung :) Bewegung :

Abbildung 4.2 Covariation Bias bei Bewegungsbildern: Anzahl der falsch positiven Antworten der drei Gruppen 


\section{Hypothese 6}

Auch die Ergebnisse des Covariation Bias werden für die CLBP-Patienten noch einmal getrennt untersucht, entsprechend den Ergebnissen des FABQ (Mittelwerte und Standardabweichungen der falsch positiven Antworten der beiden Subgruppen siehe Tabelle 4.14). Bei der Berechnung der 2 x 2 ANOVA mit Messwiederholung "Subgruppe" (Fear-Avoidance hoch/niedrig) x "Qualität" (Bewegung :-) / :) ) zeigt sich ein Haupteffekt "Qualität" ( $\mathrm{p}<0.05)$, d.h. beide Gruppen irren sich bei den negativen Bildern im Mittel häufiger in Bezug auf die Kovariation von Startle-Reiz und Bild. Es ergibt sich jedoch kein Haupteffekt "Subgruppe" und auch nicht der erwartete Interaktionseffekt. Die Subgruppe mit ausgeprägteren Fear-Avoidance Beliefs gibt also nicht häufiger irrtümlich an, dass die rückenschmerzrelevanten Bilder zusammen mit dem Startle-Reiz präsentiert wurden (s. Tabelle 4.15).

Tabelle 4.14 Covariation Bias bei Bewegungsbildern in Abhängigkeit von Fear-Avoidance: Mittelwerte und Standardabweichungen ( )

\begin{tabular}{|l|l|l|}
\hline & Fear-Avoidance hoch & Fear-Avoidance niedrig \\
\hline Bewegung $\odot ;$ & $2.28(1.6)$ & $1.83(1.85)$ \\
\hline Bewegung $: ;$ & $3.44(2.25)$ & $2.83(2.85)$ \\
\hline
\end{tabular}

Tabelle 4.15 Covariation Bias bei Bewegungsbildern in Abhängigkeit von Fear-Avoidance: Ergebnisse der Varianzanalyse

\begin{tabular}{|l|l|l|l|}
\hline & F & df & p \\
\hline Gruppe (FA hoch/ niedrig) & 0.87 & $1 / 34$ & 0.36 \\
\hline Qualität ( (; / :) & 5.47 & $1 / 34$ & $0.025 *$ \\
\hline Gruppe x Qualität & 0.32 & $1 / 34$ & 0.85 \\
\hline
\end{tabular}

${ }^{*} \mathrm{p} \leq 0.05$ 


\section{Hypothese 7}

Die oben beschriebenen Berechnungen werden analog auch für die Bilder der IAPS durchgeführt. Dabei wird angenommen, dass die Ergebnisse das in der Literatur beschriebene Bild widerspiegeln. Erwartet wird:

- ein signifikanter Unterschied in der Einschätzung der Valenz zwischen den positiven und den negativen Bildern

- eine signifikante Erhöhung der Startle-Reaktion bei der Betrachtung der negativen Bilder im Vergleich zu der Reaktion auf die positiven Bilder

- ein signifikant höherer Covariation Bias in Bezug auf die negativen Bilder im Vergleich zu den positiven Bildern, d.h. häufiger die falsch positive Angabe, dass die negativen Bilder mit dem Startle-Reiz zusammen präsentiert worden seien.

Darüber hinaus wird vorhergesagt, dass sich die drei Gruppen in allen drei Aspekten nicht voneinander unterscheiden. Zur Überprüfung dieser Annahmen werden analog zu der Berechnung der Bewegungsbilder jeweils 3 x 2 ANOVAs "Gruppe“ (CLBP, KG KS, KG GES) x "Qualität" (;)/:) mit Messwiederholung berechnet.

\section{- Valenzeinschätzung}

Für die Einschätzung der Valenz der IAPS- Bilder zeigt die Varianzanalyse wie erwartet einen hoch signifikanten Haupteffekt "Qualität" ( $\mathrm{p}<0.001)$. Das bedeutet, dass die positiven und die negativen Bilder signifikant unterschiedlich eingestuft werden. Die drei Gruppen unterscheiden sich in ihrer Einschätzung der Bilder wie vorhergesagt nicht, was sich darin zeigt, dass der Haupteffekt "Gruppe" nicht signifikant wird. Der Interaktionseffekt wird ebenfalls wie vorhergesagt nicht signifikant (Ergebnisse s. Tabelle 4.16 und 4.17). 
Tabelle 4.16 Einschätzung der Valenz der IAPS-Bilder:

Mittelwerte und Standardabweichungen ( )

\begin{tabular}{|l|l|l|l|}
\hline & CLBP & KG KS & KG GES \\
\hline IAPS $:-$ & $7.21(0.95)$ & $6.96(0.9)$ & $6.98(0.91)$ \\
\hline IAPS $: 0$ & $1.98(0.86)$ & $2.04(0.79)$ & $2.03(0.8)$ \\
\hline
\end{tabular}

Tabelle 4.17 Einschätzung der Valenz der IAPS-Bilder: Ergebnisse der Varianzanalyse

\begin{tabular}{|l|l|l|l|}
\hline & F & df & $p$ \\
\hline Gruppe (CLBP, KG KS, KG GES) & 2.42 & $2 / 69$ & 0.79 \\
\hline Qualität (:) / : ) & 1095.12 & $1 / 69$ & $<0.0001^{* * *}$ \\
\hline Gruppe x Qualität & 0.52 & $2 / 69$ & 0.6 \\
\hline
\end{tabular}

$* * * \mathrm{p} \leq 0.001$

- Startle-Reaktion

Die Varianzanalyse zeigt den vorhergesagten sehr signifikanten Haupteffekt "Qualität" ( $p<0.001$ ) sowie entsprechend der Vorhersage keinen Haupteffekt "Gruppe" und keinen Interaktionseffekt (Ergebnisse s. Tabelle 4.18 und 4.19). Alle drei Gruppen zeigen also im Mittel die vorhergesagte Erhöhung der Startle-Reaktion bei der Betrachtung der negativen Bilder der IAPS.

Tabelle 4.18 Startle-Reaktion auf IAPS-Bilder: Mittelwerte und Standardabweichungen (Adunits), nur Responder

\begin{tabular}{|l|l|l|l|}
\hline & CLBP & KG KS & KG GES \\
\hline IAPS $:-$ & $301.56(249.7)$ & $359.14(309.6)$ & $313.18(197.5)$ \\
\hline IAPS $:-$ & $358.47(290.7)$ & $402.4(335.7)$ & $332.27(181.7)$ \\
\hline
\end{tabular}


Tabelle 4.19 Startle-Reaktion auf IAPS-Bilder (nur Responder): Ergebnisse der Varianzanalyse

\begin{tabular}{|l|l|l|l|}
\hline & $\mathrm{F}$ & $\mathrm{df}$ & $\mathrm{p}$ \\
\hline Gruppe (CLBP, KG KS, KG GES) & 0.24 & $2 / 56$ & 0.78 \\
\hline Qualität (-) / : ) & 14.74 & $1 / 56$ & $0.0003^{* * *}$ \\
\hline Gruppe x Qualität & 1.16 & $2 / 56$ & 0.32 \\
\hline
\end{tabular}

$* * * \mathrm{p} \leq 0.001$

In der vorliegenden Studie waren zur Überprüfung der neutralen Reaktion Startle-Reize in den Pausen zwischen den Bildern gegeben worden, also ohne Emotionsinduktion über Bilder. Wie u.a. Bradley et al. (1999) zeigen, ist die Reaktion auf neutrale Stimuli üblicherweise in der Höhe zwischen der Reaktion auf negative und der Reaktion auf positive Stimuli zu finden. Die Reaktion der Versuchspersonen entspricht jedoch nicht den Erwartungen (s. Anhang C2).

\section{- Covariation Bias}

Wie vorhergesagt zeigt die Varianzanalyse einen sehr signifikanten Haupteffekt "Qualität" ( $\mathrm{p}<0.001)$ und keinen Haupteffekt "Gruppe“. Alle drei Gruppen geben häufiger bei den negativen IAPS-Bildern fälschlicherweise an, dass diese zusammen mit dem Startle-Reiz präsentiert worden sind (Ergebnisse s. Tabelle 4.20 und 4.21).

Tabelle 4.20 Covariation Bias bei IAPS-Bildern:Mittelwerte und Standardabweichungen ( ) der falsch positiven Antworten

\begin{tabular}{||l|l|l|l||}
\hline & CLBP & KG KS & KG GES \\
\hline IAPS $: ;$ & $2.64(2.38)$ & $2.06(1.39)$ & $1.72(1.6)$ \\
\hline IAPS $: 0$ & $3.56(2.47)$ & $3.5(2.57)$ & $2.61(1.97)$ \\
\hline
\end{tabular}


Tabelle 4.21 Covariation Bias bei IAPS-Bildern:

Ergebnisse der Varianzanalyse

\begin{tabular}{|l|l|l|l|}
\hline & $\mathrm{F}$ & $\mathrm{df}$ & $\mathrm{p}$ \\
\hline Gruppe (CLBP, KG KS, KG GES) & 1.55 & $2 / 69$ & 0.29 \\
\hline Qualität (-) / :) ) & 11.95 & $1 / 69$ & $0.0009 * * *$ \\
\hline Gruppe x Qualität & 0.31 & $2 / 69$ & 0.73 \\
\hline$* * * \mathrm{p} \leq 0.001$ & &
\end{tabular}

4.3 Überprüfung der State-Angst, der Arousal-Einschätzung und des Einflusses der Reihenfolge (Blocks) auf die Ergebnisse der Hypothesenprüfung

- $\quad$ Überprüfung der State-Angst

Zunächst soll überprüft werden, ob sich die State-Angst gemessen mit der State-Skala des STAI zwischen den Gruppen unterscheidet. Dafür wird eine einfaktorielle Varianzanalyse mit dem Faktor "Gruppe" berechnet. Tatsächlich ergibt sich ein signifikanter Effekt "Gruppe“ ( $<<0.001)$. Der post-hoc-Test (Fisher-PLSD) zeigt, dass sich die beiden Kontrollgruppen nicht voneinander unterscheiden, sich aber jeweils signifikant von der Experimentalgruppe unterscheiden. Die Mittelwerte aller drei Gruppen machen deutlich, dass die CLBP-Patienten mehr State-Angst erleben als die beiden Kontrollgruppen (s. Tabelle 4.22). Um einen etwaigen Einfluss dieses Unterschiedes auf die Startle-Reaktion zu kontrollieren, wird die Variable State-Angst bei der Berechnung der einfaktoriellen Varianzanalyse für die Differenzwerte der Reaktion auf die Bewegungsbilder (Reaktion auf negatives Bewegungsbild minus Reaktion auf positives Bewegungsbild) als Kovariate in die Berechnung eingeführt. Die Analyse zeigt keinen Einfluss dieser Kovariate. Die Tatsache, dass die CLBP-Patienten mehr State-Angst zeigen als die Probanden der beiden Kontrollgruppen hat somit keinen Einfluss auf das Ausmaß der Startle-Erhöhung bei den negativen Bewegungsbildern (s.Tabelle 4.23). 
Tabelle 4.22 State-Angst: Mittelwerte und Standardabweichungen ( )

\begin{tabular}{||l|l|l|l|}
\hline & CLBP & KG KS & KG GES \\
\hline State-Angst M /SD & $2.1(0.45)$ & $1.86(0.33)$ & $1.67(0.22)$ \\
\hline
\end{tabular}

Tabelle 4.23 Einfluss der State-Angst auf die Startle-Reaktion:

Ergebnisse der Varianzanalyse der Startle-Differenzwerte mit Kovariate State-Angst (nur Responder)

\begin{tabular}{|l|l|l|l|}
\hline & $\mathrm{F}$ & $\mathrm{df}$ & $\mathrm{p}$ \\
\hline Gruppe (CLBP, KG KS, KG GES) & 0.32 & $2 / 53$ & 0.72 \\
\hline State-Angst & 0.38 & $1 / 53$ & 0.54 \\
\hline Gruppe x State-Angst & 0.36 & $2 / 53$ & 0.7 \\
\hline
\end{tabular}

- Einschätzung des Arousals

Es soll überprüft werden, ob es Unterschiede in der Einschätzung des Arousals der Bilder zwischen den Gruppen gibt, da die Höhe des Arousals für die erfolgreiche Emotionsinduktion entscheidend sein kann (Mittelwerte und Standardabweichungen der Einschätzungen s. Tabelle 4.24).

Tabelle 4.24 Einschätzung des Arousals der Bewegungsbilder / IAPS Bilder: Mittelwerte und Standardabweichungen ( )

\begin{tabular}{|l|l|l|l|}
\hline & CLBP & KG KS & KG GES \\
\hline Bewegung $\odot ;$ & $6.57(1.4)$ & $5.93(1.2)$ & $6.51(1.1)$ \\
\hline Bewegung $:-$ & $6.31(1.4)$ & $5.82(1.1)$ & $5.68(1.6)$ \\
\hline \hline IAPS $\odot:$ & $6.54(1.4)$ & $6.34(1.1)$ & $6.72(1.1)$ \\
\hline IAPS $: 0$ & $6.51(2.7)$ & $6.75(1.7)$ & $6.95(1.9)$ \\
\hline
\end{tabular}


Die 3 x 2 ANOVA mit Messwiederholung "Gruppe“ x "Qualität" für die Bewegungsbilder ergibt einen Haupteffekt "Qualität" ( $p<0.05)$, d.h. die negativen Bilder werden bezüglich ihres Arousal-Wertes etwas geringer eingestuft als die positiven Bilder. Einen Haupteffekt "Gruppe“ gibt es nicht, ebenso wenig einen Interaktionseffekt. Bei der Einschätzung der IAPS-Bilder zeigen sich weder Haupteffekte noch ein Interaktionseffekt (s. Tabelle 4.25).

Tabelle 4.25 Einschätzung des Arousals der Bewegungsbilder/ IAPS Bilder:

Ergebnisse der Varianzanalysen

\begin{tabular}{|l|l|l|l|l|}
\hline & & $\mathrm{F}$ & $\mathrm{df}$ & $\mathrm{p}$ \\
\hline \multirow{3}{*}{ Bewegung } & Gruppe (CLBP, KG KS, KG GES) & 1.85 & $2 / 69$ & 0.17 \\
\cline { 2 - 5 } & Qualität (:) / : ) & 4.28 & $1 / 69$ & $0.042^{*}$ \\
\cline { 2 - 5 } & Gruppe x Qualität & 1.19 & $2 / 69$ & 0.31 \\
\hline \multirow{3}{*}{ IAPS } & Gruppe (CLBP, KG KS, KG GES) & 0.3 & $2 / 69$ & 0.74 \\
\cline { 2 - 5 } & Qualität (:) / : ) & 0.81 & $1 / 69$ & 0.37 \\
\cline { 2 - 5 } & Gruppe x Qualität & 0.39 & $2 / 69$ & 0.69 \\
\hline
\end{tabular}

$*=\mathrm{p} \leq 0.05$

Um zu überprüfen, ob es Unterschiede in der Arousal-Bewertung der Bewegungsbilder und der IAPS Bilder gibt, wurde jeweils eine 3 x 2 ANOVA mit Messwiederholung "Gruppe" (CLBP, KG KS, KG GES) x "Quelle" (IAPS, Bewegung) gerechnet, sowohl für die positiven Bilder beider Quellen als auch für die negativen Bilder. Zwischen den positiven Bildern zeigen sich keine Unterschiede zwischen der Bewertung der IAPS und der Bewegungsbilder, es gibt weder Haupteffekte, noch einen Interaktionseffekt. Für die negativen Bilder zeigt sich ein hoch signifikanter Haupteffekt "Quelle“ (p<0.001), d.h. die Bilder der IAPS werden als stärker erregend eingeschätzt als die Bewegungsbilder. Es zeigt sich ebenfalls ein, wenn auch schwächerer Interaktionseffekt $(\mathrm{p}<0.05)$; die Probanden der CLBP-Gruppe schätzen die negativen Bewegungsbilder als stärker erregend ein als die beiden anderen Gruppen (s. Tabelle 4.26 und Abbildung 4.3).

Um einen direkten Vergleich der Bewertung der negativen IAPS-Bilder und der negativen Bewegungsbilder der CLBP-Patienten zu erhalten, wird der Unterschied 
zwischen den Einschätzungen der Experimentalgruppe mittels eines t-Test für abhängige Stichproben betrachtet. Es zeigt sich, dass die CLBP-Patienten die negativen IAPS- und die Bewegungsbilder nicht unterschiedlich einschätzen, der Effekt "Quelle" in der Varianzanalyse geht also auf die beiden anderen Gruppen zurück (s. Tabelle 4.26 und Abbildung 4.3).

Tabelle 4.26 Einschätzung des Arousals der Bewegungsbilder und der IAPS Bilder: Ergebnisse der Varianzanalysen und des t-Tests

\begin{tabular}{|c|l|l|l|l|}
\hline & & $\mathrm{F} / \mathrm{t}$ & $\mathrm{df}$ & $\mathrm{p}$ \\
\hline $\begin{array}{c}\text { Bewegung/IAPS } \\
(;\end{array}$ & Gruppe (CLBP, KG KS, KG GES) & 1.07 & $2 / 69$ & 0.35 \\
\cline { 2 - 5 } & Quelle (Bewegung / IAPS) & 2.25 & $1 / 69$ & 1.14 \\
\cline { 2 - 5 } & Gruppe x Quelle & 1.02 & $2 / 69$ & 0.37 \\
\hline $\begin{array}{c}\text { Bewegung/IAPS } \\
*\end{array}$ & Gruppe (CLBP, KG KS, KG GES) & 16.85 & $2 / 69$ & 0.95 \\
\cline { 2 - 5 } & Quelle (Bewegung / IAPS) & 4.28 & $1 / 69$ & $0.0001^{* * *}$ \\
\cline { 2 - 5 } & Gruppe x Quelle & 3.22 & $2 / 69$ & $0.046^{*}$ \\
\hline Nur CLBP & IAPS : / Bewegung $:$ & 0.81 & $1 / 35$ & 0.42 \\
\hline
\end{tabular}

*** $\mathrm{p} \leq 0.001 \quad * \mathrm{p} \leq 0.05$

Arousal

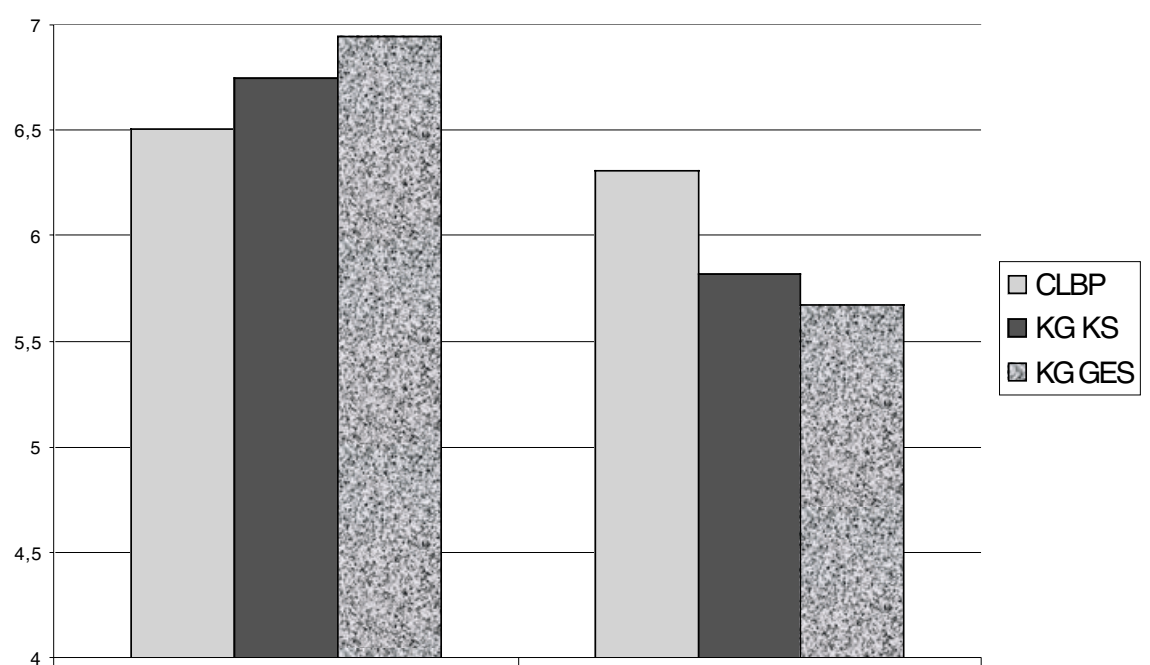

IAPS :- Bewegung :

Abbildung 4.3 Arousaleinschätzung der negativen Bilder der drei Gruppen 
- Überprüfung der Auswirkung der Reihenfolge (Blocks)

Die Ergebnisse der Analysen für die Einschätzung der Valenz bzw. des Arousals der Bewegungsbilder, die Startle-Reaktion und den Covariation Bias werden möglicherweise davon beeinflusst, ob die Probanden erst die Bewegungsbilder und dann die IAPS-Bilder gesehen haben oder umgekehrt (Block 1 oder Block 2). Darum soll dieser Effekt geprüft werden. Dafür wird für alle weiter oben geprüften abhängigen Variablen (Evaluation von Valenz und Arousal, Startle-Reaktion und Covariation Bias) jeweils eine 2 × 2 ANOVA "Block“ (1/2) x "Qualität"(:)/ :) gerechnet.

\section{- Bilderevaluation}

Bei der Überprüfung des Einflusses der Blocks auf die Evaluation der Valenz und des Arousals der Bilder zeigt die ANOVA weder einen signifikanten Haupteffekt "Block", noch einen Interaktionseffekt (s. Tabelle 4.27). Auf eine weitere Analyse der Daten wird also verzichtet.

Tabelle 4.27 Einfluss der Blocks auf die Valenz- und Arousal- der Bewegungsbilder: Ergebnisse der Varianzanalysen

\begin{tabular}{|l|l|l|l|l|}
\hline & & F & df & p \\
\hline \multirow{4}{*}{ Valenz } & Block (1 /2)) & 1.004 & $1 / 70$ & 0.32 \\
\cline { 2 - 5 } & Block x Qualität & 2.52 & $1 / 70$ & 0.12 \\
\hline \multirow{3}{*}{ Arousal } & Block (1 /2) & 0.19 & $1 / 70$ & 0.66 \\
\cline { 2 - 5 } & Block x Qualität & 0.71 & $1 / 70$ & 0.4 \\
\hline
\end{tabular}

\section{- Startle-Reaktion:}

Die ANOVA für den Effekt der Blocks auf die Startle-Reaktion ergibt einen signifikanten Haupteffekt "Block“ $(\mathrm{p}<0,05)$, jedoch keinen Interaktionseffekt (s. Tabelle 4.28). Wie in Tabelle 4.29 mit den Mittelwerten der Startle-Reaktion deutlich wird, zeigen sich in Block 2 niedrigere Werte als in Block 1. 
Tabelle 4.28 Einfluss der Blocks auf die Startle-Reaktion auf die Bewegungsbilder (nur Responder):

Ergebnisse der Varianzanalysen

\begin{tabular}{|l|l|l|l|}
\hline & $\mathrm{F}$ & $\mathrm{df}$ & $\mathrm{p}$ \\
\hline Block $(1 / 2))$ & 5.56 & $1 / 57$ & 0.02 \\
\hline Block x Qualität & 0.41 & $1 / 57$ & 0.52 \\
\hline
\end{tabular}

Tabelle 4.29 Einfluss der Blocks auf die Startle-Reaktion auf die Bewegungsbilder (nur Responder): Mittelwerte und Standarabweichungen ( )

\begin{tabular}{|l|l|l|l|l|}
\hline & & CLBP & KG KS & KG GES \\
\hline Block 1 & Bewegung $:-$ & $500.3(417)$ & $485.5(439.4)$ & $295(192.5)$ \\
\cline { 2 - 5 } & Bewegung $:-$ & $536.6(359.9)$ & $468.5(376.2)$ & $315.6(236.7)$ \\
\hline Block 2 & Bewegung $:-$ & $243.9(166.5)$ & $317.5(318.8)$ & $295.2(218.5)$ \\
\cline { 2 - 5 } & Bewegung $:-$ & $248.4(166.4)$ & $279.1(253.4)$ & $316(187.4)$ \\
\hline
\end{tabular}

Um zu überprüfen, ob die Hypothese 1 möglicherweise für einen der beiden Blocks bestätigt werden kann, wird die ANOVA "Gruppe“ x "Qualität" mit Messwiederholung für die Gruppen noch einmal getrennt nach den Blocks berechnet.

Bei der getrennten Analyse der beiden Blocks zeigen sich weder Haupteffekte noch der in der Hypothese erwartete Interaktionseffekt (Tabelle 4.30). Das heißt also, dass die Rückenschmerzpatienten, unabhängig von der Frage, ob sie erst die Bewegungs- oder die IAPS-Bilder gesehen haben, bei der Betrachtung der rückenschmerzrelevanten Bewegungsbilder keine Erhöhung der Startle-Reaktion zeigen. 
Tabelle 4.30 Startle-Reaktion auf Bewegungsbilder getrennt nach Blocks (nur Responder): Ergebnisse der Varianzanalysen

\begin{tabular}{|l|l|l|l|l|}
\hline & & F & df & p \\
\hline \multirow{3}{*}{ Block 1 } & Gruppe & 0.86 & $1 / 26$ & 0.43 \\
\cline { 2 - 5 } & Qualität & 0.21 & $1 / 26$ & 0.64 \\
\cline { 2 - 5 } & Gruppe x Qualität & 0.34 & $1 / 26$ & 0.72 \\
\hline \multirow{3}{*}{ Block 2 } & Gruppe & 0.25 & $1 / 27$ & 0.78 \\
\cline { 2 - 5 } & Qualität & 0.03 & $1 / 27$ & 0.85 \\
\cline { 2 - 5 } & Gruppe x Qualität & 0.65 & $1 / 27$ & 0.53 \\
\hline
\end{tabular}

\section{- Covariation Bias}

Bei der Berechnung des Einflusses der Blocks auf den Covariation Bias bei den Bewegungsbilder ergibt die ANOVA einen signifikanten Haupteffekt "Block" $(\mathrm{p}<0.001)$ und keinen Interaktionseffekt (Tabelle 4.32). Bei der Analyse des Covariation Bias zeigt sich also ein signifikanter Unterschied in der Häufigkeit der falschen Annahme, dass die Bewegungsbilder mit dem unangenehmen Reiz zusammen präsentiert wurden, in Abhängigkeit davon, ob die Probanden zuerst die Bewegungsbilder sahen oder erst die Bilder der IAPS. Da die Probanden beim zweiten Durchgang bereits wussten, dass sie nach dem Zusammenhang der Bilder mit dem Ton gefragt wurden, sind die entscheidenden Ergebnisse hier die des Blocks 1.

Tabelle 4.31 Einfluss der Blocks auf den Covariation Bias bei den Bewegungsbildern: Mittelwerte und Standardabweichungen ( )

\begin{tabular}{|l|l|l|l|l|}
\hline & & CLBP & KG KS & KG GES \\
\hline Block 1 & Bewegung $:-$ & $2.55(1.58)$ & $3.22(1.64)$ & $3.1(1.9)$ \\
\cline { 2 - 5 } & Bewegung $:-$ & $4.33(2.54)$ & $1.89(1.17)$ & $3.11(1.61)$ \\
\hline Block 2 & Bewegung $:-$ & $1.55(1.76)$ & $3(1.93)$ & $0.67(0.7))$ \\
\cline { 2 - 5 } & Bewegung $\odot-$ & $1.94(1.98)$ & $1.89(1.53)$ & $1.77(2.33)$ \\
\hline
\end{tabular}


Tabelle 4.32 Einfluss der Blocks auf den Covariation Bias bei den Bewegungsbildern: Ergebnisse der Varianzanalysen

\begin{tabular}{|l|l|l|l|}
\hline & $\mathrm{F}$ & $\mathrm{df}$ & $\mathrm{p}$ \\
\hline Block $(1 / 2))$ & 15.56 & $1 / 70$ & $0.0002 * * *$ \\
\hline Block x Qualität & 0.37 & $1 / 70$ & 0.54 \\
$* * * \mathrm{p} \leq 0.001$ & & &
\end{tabular}

Die ANOVA "Gruppe“ x "Qualität" mit Messwiederholung für die Gruppen wird getrennt nach den Blocks berechnet. Aufgeführt werden hier nur die Eregebnisse für den Block 1 (Ergebnisse für Block 2 siehe Anhang C3). Es zeigt sich kein signifikanter Haupteffekt, jedoch ein Interaktionseffekt. Wie in Abbildungen $4.4 \mathrm{zu}$ sehen ist, zeigen die CLBP-Patienten den Covariation Bias in die erwartete Richtung (mehr falsch positive Antworten bei den rückenschmerzrelevanten Bildern). Die Kopfschmerzpatienten zeigen einen höheren Bias bei den positiven Bildern (s. Tabelle 4.33)

Tabelle 4.33 Covariation Bias bei Bewegungsbildern in Block 1 Ergebnisse der Varianzanalyse

\begin{tabular}{|l|l|l|l|l|}
\hline & & F & df & p \\
\hline \multirow{3}{*}{ Block 1 } & Gruppe & 1.39 & $1 / 33$ & 0.26 \\
\cline { 2 - 6 } & Qualität & 0.96 & $1 / 33$ & 0.76 \\
\cline { 2 - 5 } & Gruppe x Qualität & 4.16 & $1 / 33$ & $0.02 *$ \\
\hline
\end{tabular}

$* \mathrm{p} \leq 0.05$ 
falsch positive

Antworten

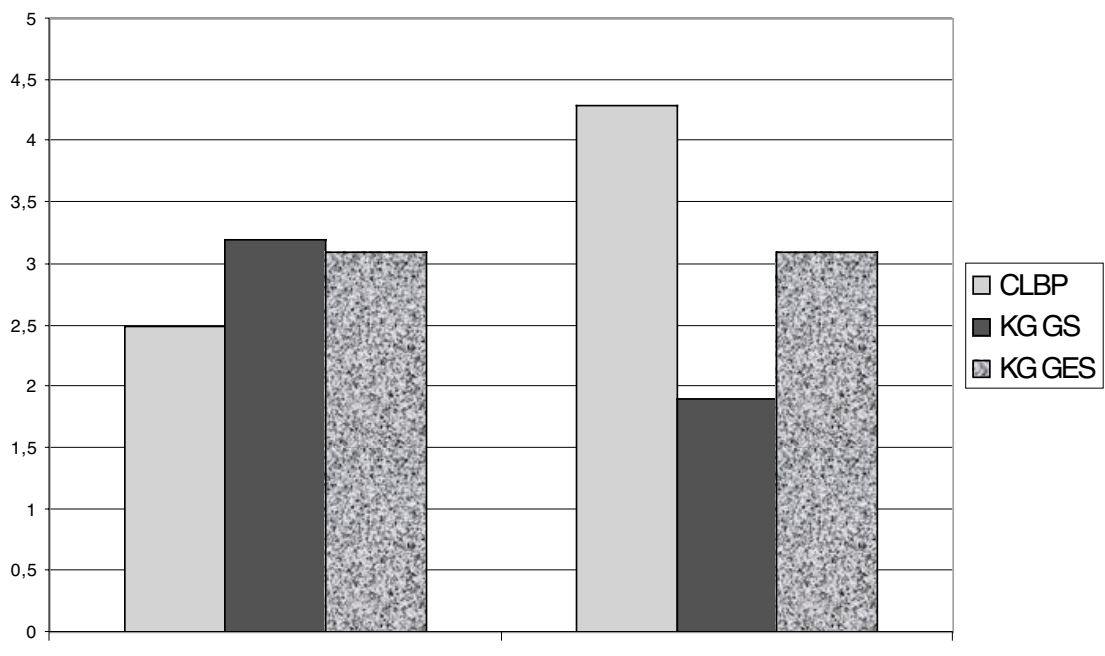

Bewegung $(;) \quad$ Bilder Bewegung $:$

Abbildung 4.4 Covariation Bias bei den Bewegungbildern in Block 1

4.4 Analyse der Fragebogen und ihrer Zusammenhänge

Sowohl für den SAB als auch für den SEB und den ABB wird die interne Konsistenz berechnet. Beim SAB ergibt sich ein Wert für Cronbachs Alpha von 0.92. Da alle Items ausreichend mit der Gesamtskala korrelieren, werden alle Items in die weitere Berechnung hinein genommen. Die Berechnung einer Faktorenanalyse mit VarimaxRotation zeigt drei Faktoren mit einem Eigenwert über 1. Da jedoch alle Items ausreichend hoch auf dem ersten Faktor laden und auch der Scree-Test eine einfaktorielle Lösung nahe legt, wird diese Lösung angenommen. Der Faktor klärt 49\% Varianz auf. Die bei der Entwicklung des Fragebogens theoretisch angenommenen zwei Faktoren "Worry" und "Emotionality" spiegeln sich in der Faktorenstruktur der vorliegenden Daten nicht wieder.

Bei der Berechnung der internen Konsistenz des SEB verringert ein Item die Konsistenz des Fragebogens und wird deshalb aus den weiteren Berechnungen herausgenommen. Mit nunmehr 10 Items hat der Fragebogen eine interne Konsistenz von 0.87 (Cronbachs Alpha). Die Faktorenanalyse mit Varimax-Rotation ergibt zwei Faktoren mit 
Eigenwerten über 1. Auch hier wird aufgrund der ausreichend hohen Ladungen auf dem ersten Faktor und den Hinweisen des Scree-Tests eine einfaktorielle Lösung angenommen. Der Faktor klärt 46\% der Varianz auf (genaue Ergebnisse der Faktorenanalysen siehe Anhang C4/5).

Die Itemanalyse des ABB zeigt, dass das Item "Sicherheit" nur gering mit den anderen Items des Fragebogens korreliert. Aus den weiteren Berechnungen wird es deshalb herausgenommen. Mit den verbleibenden neun Items hat der Fragebogen eine interne Konsistenz von 0.88 (Cronbachs Alpha).

\section{- Zusammenhänge des Fear-Avoidance Beliefs Questionnaire mit den anderen} Fragebögen

Zunächst sollen die Zusammenhänge des Fear-Avoidance Beliefs Questionnaire mit dem Schmerz-Angst-Bogen, dem Schmerz-Einstellungsbogen, dem Arbeits-BeschreibungsBogen und den Skalen State und Trait des State-Trait-Anxiety Inventory überprüft werden. Dazu werden bivariate Korrelationen nach Pearson berechnet und zweiseitig auf Signifikanz getestet. Tabelle 4.34 zeigt die Ergebnisse. Da der FABQ nur den CLBPPatienten vorgelegt wurde, liegen der Berechnung Daten von 36 Personen zugrunde, bzw. für den ABB Daten von 33 Personen (drei Personen der Experimentalstichprobe hatten aktuell keine Arbeitsstelle) Um die Zusammenhänge des FABQ mit anderen Verfahren differenzierter beleuchten zu können, wird außer einem Gesamtwert für den FABQ auch jeweils ein Wert für die drei Faktoren nach Pfingsten et al (2000) berechnet: "Ursache Arbeit", "Prognose Arbeit" und "Aktivität". Bei der Betrachtung der Korrelationen gilt es zu beachten, dass bei den Fragebögen SAB und SEB ein niedriger Wert eine hohe Zustimmung bedeutet, während bei den anderen Fragebögen ein niedriger Wert geringe Zustimmung bedeutet. Es werden also negative Korrelationen erwartet. 
Tabelle 4.34 Korrelationen nach Pearson der FABQ-Werte (Gesamt und Unterfaktoren) mit den Werten des SAB, SEB, ABB, STAI-State und STAI-Trait

\begin{tabular}{|l|l|l|l|l|}
\hline & FABQ & Ursache Arbeit & Prognose Arbeit & Aktivität \\
\hline SAB & -.222 & -.144 & -.153. & -.316 \\
\hline SEB & -.314 & -.292 & -.190 & $-.357^{*}$ \\
\hline ABB & .322 & $.420^{*}$ & .276 & .074 \\
\hline STAI-State & $.376^{*}$ & $.333^{*}$ & .301 & $.375^{*}$ \\
\hline STAI-Trait & $.388^{*}$ & $.361^{*}$ & .268 & $.364^{*}$ \\
\hline
\end{tabular}

$*=\mathrm{p}<0,05$

Wie aus Tabelle 4.34 deutlich wird, zeigt der Gesamtwert des FABQ weder einen bedeutsamen Zusammenhang zum SAB noch zum SEB oder zum ABB. AusZusammenhänge ergeben sich dagegen zu den beiden Skalen des STAI, also sowohl zur Trait Anxiety als auch zur State Anxiety. Der FABQ-Faktor "Ursache Arbeit" korreliert ebenfalls mit diesen beiden Skalen, zusätzlich jedoch auch noch mit dem ABB. Der Faktor "Prognose Arbeit" zeigt keinerlei Zusammenhänge zu den übrigen Fragebögen. Der Faktor “Aktivität" zeigt einen Zusammenhang zum SEB und zur Trait bzw. zur State Anxiety. Alle diese Korrelationen werden zwar auf dem 5\%-Niveau signifikant, haben aber maximal eine gemeinsame Varianz von knapp 18\%. Der stärkste Zusammenhang zeigt sich zwischen dem Faktor "Ursache Arbeit" und dem ABB. Erwartungsgemäß zeigen die Faktoren des FABQ untereinander hohe Korrelationen (max. $r=0.83$, Ergebnisse siehe Anhang C6).

\section{- Zusammenhänge zwischen SAB / SEB und ABB bzw. STAI}

Um die Zusammenhänge des SAB und des SEB untereinander bzw. ihren jeweiligen Zusammenhang zum ABB und zu den beiden Skalen des STAI zu überprüfen, werden ebenfalls bivariate Korrelationen berechnet. Die Ergebnisse zeigt Tabelle 4.35. Der Schmerz-Angst-Bogen zeigt relativ starke Zusammenhänge zu den beiden Skalen des STAI, also sowohl zur State (27\% gemeinsame Varianz) als auch zur Trait Anxiety (38\% gemeinsame Varianz). Es zeigt sich jedoch auch ein, wenn auch schwächerer Zusammenhang zum ABB. Die Scores des SAB und SEB korrelieren ebenfalls signifikant - mittelhoch - miteinander mit einer gemeinsamen Varianz von $18 \%$. Der SEB zeigt zudem noch einen Zusammenhang zur Trait-Skala des STAI. 
Tabelle 4.35 Korrelationen nach Pearson der Werte des SAB/SEB mit den Werten des ABB, STAI-State und STAI-Trait

\begin{tabular}{|l|l|l|}
\hline & SAB & SEB \\
\hline SAB & & \\
\hline SEB & $.428^{* *}$ & \\
\hline ABB & $-.332^{*}$ & -.258 \\
\hline State Anxiety & $-.524^{* *}$ & -.036 \\
\hline Trait Anxiety & $-.617^{* *}$ & $-.248^{*}$ \\
\hline
\end{tabular}

$*=\mathrm{p}<0.05$

$* *=\mathrm{p}<0.01$

- Analyse potentieller Unterschiede der Scores des SAB /SEB zwischen den Gruppen

Um zu überprüfen, ob die CLBP-Patienten auf dem SAB und dem SEB höhere Werte zeigen als die Personen der beiden Kontrollgruppen, wird jeweils eine einfaktorielle Varianzanalyse mit dem Faktor "Gruppe" (CLBP, KG KS, KG GS) gerechnet (Mittelwert und Standardabweichung der beiden Fragebögen s. Tab 4.36).

Tabelle 4.36 Ergebnisse der Fragebögen SAB, SEB:

Mittelwerte und Standardabweichungen ( )

\begin{tabular}{|l|l|l|l|}
\hline & CLBP & KG KS & KG GES \\
\hline SAB & $3.6(1.05)$ & $3.6(0.73)$ & $4.0(0.77)$ \\
\hline SEB & $3.7(0.67)$ & $2.8(0.68)$ & $3.6(0.67)$ \\
\hline
\end{tabular}

Für den SAB zeigt die Varianzanalyse keine signifikanten Unterschiede zwischen den Gruppen (s. Tabelle 4.37). Alle drei Gruppen zeigen also in gleichem Maße Angst bei Schmerzen. Die Varianzanalyse für den SEB zeigt einen sehr signifikanten Gruppenunterschied $(\mathrm{p}<0.001)$. Der post-hoc-Test (Fisher PLSD) ergibt sehr signifikante Unterschiede zwischen der Kopfschmerzgruppe und den beiden anderen Gruppen, wohingegen sich die Experimentalgruppe und die gesunde Kontrollgruppe nicht voneinander unterscheiden (s. Tabelle 4.38). Bei der Betrachtung der Mittelwerte 
der drei Gruppen in Tabelle 4.36 wird deutlich, dass entgegen der Annahme nicht die Rückenschmerzpatienten sondern die Kopfschmerzpatienten stärker die Einstellung bekunden, dass Menschen mit Schmerzen sich schonen sollten bzw. Aktivitäten vermeiden.

Tabelle 4.37 SAB, SEB: Ergebnisse der Varianzanalyse mit dem Faktor "Gruppe“

\begin{tabular}{|l|l|l|l|}
\hline & F & df & $p$ \\
\hline SAB (CLBP, KG KS, KG GES) & 1.22 & $2 / 69$ & 0.3 \\
\hline SEB (CLBP, KG KS, KG GES) & 10.7 & $2 / 69$ & $<0.001$ \\
\hline
\end{tabular}

Tabelle 4.38 Unterschiede im SEB zwischen den Gruppen: Ergebnisse des Post-hocTests (Fisher PLSD)

\begin{tabular}{|l|l|l|l|}
\hline & mittlere Diff. & kritische Diff. & $\mathrm{p}$ \\
\hline CLBP, KG GES & 0.071 & 0.39 & 0.72 \\
\hline CLBP, KG KS & 0.87 & 0.39 & $<0.0001 * * *$ \\
\hline KG GES, KG KS & 0.8 & 0.48 & $0.0007 * * *$ \\
\hline
\end{tabular}

$* * * \mathrm{p}<0.001$

Da sich bei der Analyse der Daten für die Experimentalgruppe und die beiden Kontrollgruppen doch einige Unterschiede zwischen den beiden Kontrollgruppen gezeigt haben, wird darauf verzichtet, wie ursprünglich geplant, die Daten dieser beiden Gruppen zusammenzufassen . 


\section{Diskussion}

5.1 Untersuchung der Konzepte Bewegungsangst und Fear-Avoidance Beliefs unter Verwendung des Startle-Paradigmas

Die vorliegende Studie untersucht die Bedeutung von Bewegungsangst bei Patienten mit chronischen Rückenschmerzen. Dabei wird der Frage nachgegangen, ob CLBPPatienten, wenn sie mit rückenschmerzrelevanten Bewegungen konfrontiert werden, im Experiment tatsächlich Bewegungsangst erleben. Um dies zu überprüfen, schätzen die Probanden der drei Gruppen (CLBP, KG KS, KG GES) die Valenz der rückenschmerzrelevanten Bilder ein. Zudem wird die Stärke der Startle-Reaktion auf die Bilder erhoben, die einen Hinweis auf die motivational/emotionale Situation gibt, in der sich eine Person befindet.

Es wird davon ausgegangen, dass Patienten Bilder von rückenschmerzrelevanten Bewegungen als aversiver einstufen als gesunde Probanden bzw. Probanden mit Kopfschmerzen, für die Bewegungsangst nicht relevant sein sollte. Darüber hinaus wird erwartet, dass die CLBP-Patienten, wenn sie mit diesen Bildern konfrontiert werden und sich vorstellen, die Bewegungen auch auszuführen, eine Erhöhung der Startle-Reaktion zeigen.

Wie erwartet schätzen die CLBP-Patienten die rückenschmerzrelevanten Bilder signifikant negativer ein als die beiden anderen Gruppen. In der Bewertung der positiven Bilder unterscheiden sich die drei Gruppen nicht. Da diese Bilder ausgesprochen angenehme Bewegungen bzw. Haltungen darstellen (wie z. B. das Liegen im Entspannungssessel), ist das nicht überraschend. Auch die beiden Kontrollgruppen bewerten die rückenschmerzrelevanten Bilder deutlich negativer als die positiven Bilder, d.h. die dargestellten Bewegungen werden generell als relativ unangenehm empfunden. Dennoch zeigt sich der erwartete Unterschied in der Bewertung zwischen den Gruppen, d.h. subjektiv empfinden die CLBP-Patienten die Bilder als deutlich aversiver als die beiden anderen Gruppen. Die Bewertung der negativen Bilder unterscheidet sich jedoch nicht wie angenommen zwischen den Patienten mit ausgeprägten und weniger ausgeprägten Fear-Avoidance Beliefs. Die Bewertung der Valenz der rückenschmerzrelevanten Bilder ist also offenbar von den Fear-Avoidance Beliefs unabhängig. 
Obwohl die CLBP-Patienten die Bewegungen subjektiv als unangenehmer bewerten, zeigen sie jedoch bei der Startle-Ableitung keine dem entsprechende Reaktion. Konfrontiert mit den Bildern der rückenschmerzrelevanten Bewegungen reagieren sie nicht, wie erwartet, mit einer Erhöhung des Startle-Reflexes. Auch wenn Patienten mit ausgeprägten und weniger ausgeprägten Fear-Avoidance Beliefs getrennt betracht werden, ergibt sich nicht der erwartete Effekt: beide Subgruppen unterscheiden sich voneinander nicht in ihrer Startle-Reaktion auf die Bewegungsbilder. Die Hälfte der Probanden sah zuerst die Bewegungsbilder (Block 1), die andere Hälfte zuerst die Bilder der IAPS (Block 2). Auch bei der getrennten Betrachtung der Blocks lässt sich die Hypothese nicht bestätigen: weder in Block 1, in dem Habituation noch keine so große Bedeutung hat, noch in Block 2 zeigt sich der angenommene Unterschied der StartleReaktion auf die Bewegungsbilder. Somit kann ausgeschlossen werden, dass der fehlende Effekt in den über den Gesamtversuch aggregierten Daten auf eine Habituation im Verlauf der Bilderdarbietung zurückzuführen ist.

Für die Befunde bieten sich verschiedene Erklärungen an. Zunächst stellt sich die Frage, ob das Startle-Paradigma in der vorliegenden Untersuchung experimentell korrekt umgesetzt wurde. Um dies überprüfen zu können, wurden nicht nur die selbst erstellten Bilder verwendet, sondern ebenfalls Bilder der IAPS, auf die typische StartleReaktionsmuster gut dokumentiert sind. Die nach den Daten der Literatur zu erwartende Befundstruktur in Bezug auf die IAPS-Bilder wird in dieser Studie repliziert. Bei allen drei Gruppen zeigen sich signifikante Unterschiede in der Startle-Reaktion auf positive und negative Bilder der IAPS. Die technisch-methodische Anlage des Versuchs scheint also nicht für den fehlenden Effekt verantwortlich zu sein.

Eine Ursache dafür, warum die Emotionsinduktion mit den IAPS-Bildern gelungen ist und mit den Bewegungsbildern offenbar nicht, könnte darin liegen, dass die Bewertung der Valenz der negativen Bewegungsbilder nicht ausgeprägt negativ genug ist, bzw. die Einschätzung des Arousals nicht hoch genug. Tatsächlich werden in den meisten Studien mit IAPS-Bildern negative Bilder verwendet, deren Valenzeinschätzungen den Wert von 2.5 nicht überschreiten (Bradley und Lang, 1994, 1= sehr negativ, $9=$ sehr positiv). In der vorliegenden Studie liegt die mittlere Einschätzung der aversiven Bewegungsbilder durch die CLBP-Patienten bei 3.2. Da die verwendeten IAPS-Bilder Motive von schwer verletzen Menschen, Unfällen oder ähnlich erschreckenden Situationen zeigen, ist dieser Unterschied nicht verwunderlich. Selbst wenn Rückenschmerzpatienten bestimmte Bewegungen als aversiv empfinden, wird der Anblick schwer verletzter Menschen sicher 
noch eine größere innere Abwehrreaktion hervorrufen. Dass sich Unterschiede in der Startle-Reaktion jedoch auch zeigen lassen, wenn die Valenzeinschätzungen nicht so niedrig sind wie bei den hier verwendeten IAPS-Bildern zeigt Mauler (1997). Sie fand bei der Untersuchung von Bulimie-Patientinnen eine deutliche Erhöhung der StartleReaktion beim Anblick von Bildern mit Nahrungsmitteln, obwohl die Valenz dieser Bilder von den Patientinnen nicht so niedrig eingeschätzt wurde, wie die zum Vergleich verwendeten IAPS-Bilder. Es kann also davon ausgegangen werden, dass es grundsätzlich möglich gewesen wäre, auch mit Bildern mit Valenz-Werten, wie sie hier vorliegen, eine Startle-Erhöhung hervorzurufen.

Das Argument, dass möglicherweise der Arousal-Wert der Bilder nicht ausreicht, um eine Reaktion hervorzurufen, kann dadurch entkräftet werden, dass sich die CLBPPatienten anders als die Personen der Kontrollgruppen nicht signifikant in ihrer ArousalEinschätzung der IAPS-Bilder und der Bewegungs-Bilder unterscheiden, sie ist also für die Bewegungsbilder ähnlich hoch wie für die Bilder der IAPS. Für beide Bilderquellen liegen zudem die Werte der Einschätzung über 6 und damit über dem Wert, der nach Bradley et al. (1999) mindestens erreicht sein sollte, um eine zuverlässige Veränderung der Startle-Reaktion hervorrufen zu können. Insgesamt kann es somit zumindest als unwahrscheinlich angesehen werden, dass Aspekte des Bildmaterials in Bezug auf die Einschätzung der Valenz und des Arousals eine Bestätigung der Hypothese über die Startle-Reaktion verhindert haben.

Auffallend ist in dieser Untersuchung der hohe Anteil der Personen, die überhaupt keine Startle-Reaktion zeigen. In der Literatur wird dieser Effekt nicht ausführlich diskutiert. Eine mögliche Erklärung ist die Tatsache, dass in vielen Untersuchungen zur StartleReaktion die Probanden vergleichsweise jünger waren, insbesondere in den Studien mit Studierenden als Probanden. In der vorliegenden Studie sind die Probanden im Mittel über vierzig Jahre alt. Ob das Alter auf die generelle Startle-Reaktion einen Einfluss hat, ist bisher bei Menschen nicht systematisch untersucht. Allerdings zeigen Varty, Hauger und Geyer (1998), dass die Reaktivität beim Startle-Reflex von Ratten mit zunehmenden Alter deutlich abnimmt. Insofern wäre es möglich, dass auch bei Menschen in höherem Alter der Prozentsatz derer, die keine Startle-Reaktion zeigen, ansteigt. Da die NonResponder bei der Überprüfung der Hypothesen zur Startle-Reaktion aus der Analyse ausgeschlossen wurden, werden die Ergebnisse der Untersuchung durch sie nur insofern beeinflusst, als die Größe der Stichprobe dadurch verringert wird. Inwieweit eine 
Untersuchung des Startle-Reflexes mit älteren Probanden in ihrer generellen Validität eingeschränkt ist, lässt sich ohne systematische Studien zu dieser Frage nicht klären. Um die ausbleibende Startle-Erhöhung bei den negativen Bewegungsbildern zu erklären, könnte man auch die Frage stellen, ob durch das Betrachten der Bilder und die Vorstellung, die Bewegung selbst auszuführen, überhaupt Angst vor Bewegungen ausgelöst worden ist. Dass es grundsätzlich möglich ist, eine Potenzierung der Schreckreaktion allein durch die Vorstellung von phobischen Reizen auszulösen, zeigen Hamm, Gerlach, Globisch und Vaitl (1992). Sie konnten eine erhöhte Schreckreaktion ebenso nachweisen, wenn sich die Probanden (Schlangen- bzw. Spinnenphobiker) die phobierelevanten Reize nur vorstellten, wie wenn sie Bilder der entsprechenden Reize betrachteten. Da hier beide Vorgehensweisen kombiniert wurden, kann man grundsätzlich davon ausgehen, dass es möglich ist, dadurch Angst auszulösen. Allerdings lässt sich nicht überprüfen, wie intensiv sich die Probanden wirklich vorgestellt haben, die Bewegungen auszuführen. Obwohl die Bewegungen als aversiv bewertet werden, könnte ihre Betrachtung dennoch ohne Angst erfolgen, da die Patienten wissen, dass sie die Bewegungen nicht wirklich ausführen müssen. Um diese Problematik zu umgehen, wäre es möglicherweise sinnvoll gewesen, den Probanden zu sagen, dass sie die Bewegungen später tatsächlich durchführen müssen. Zudem sind die Vorstellungen bei der Betrachtung statischer Bilder, gerade wenn es um Bewegungen geht, eventuell zu wenig deutlich und anschaulich. Die Darbietung eines Videofilms statt der Präsentation der Bilder wäre insofern eine Alternative gewesen. Vlaeyen et al. (1999) haben in ihrer Untersuchung zur Wirkung von Bewegungsangst auf die muskuläre Reaktivität genau dieses Vorgehen gewählt: sie zeigten Videofilme von Bewegungen und sagten den Probanden, dass sie die Bewegungen später ausführen müssten. Die Personen auf den Filmen zeigten bei den rückenschmerzrelevanten Bildern auch deutliche Schmerzreaktionen wie Grimassieren oder Stöhnen, was sicher einen zusätzlichen Einfluss hat. Dennoch konnten auch Vlaeyen et al. nicht den Effekt von Bewegungsangst auf die muskuläre Spannung zeigen, den sie erwartet hatten. Bei einer Nachbefragung ihrer Probanden wurde deutlich, dass möglicherweise eine Reihe von Personen die Bewegungsangst kognitiv vermieden hatte, indem sie sich einfach vornahmen, die Bewegung doch nicht auszuführen. Es ist nicht auszuschließen, dass kognitive Vermeidung der Vorstellung, die Bewegungen auszuführen, in der vorliegenden Studie ebenfalls eine Rolle gespielt hat. Da die Patienten nach der Untersuchung nicht systematisch befragt wurden, liegen dazu jedoch keine Daten vor. 
In vielen Studien zum Startle-Reflex werden auch Daten über Reaktionen auf neutrale Reize erhoben. Zum Teil wird dies über neutrale Bilder erreicht, teilweise wird jedoch auch in den Pausen zwischen den Bildern ein Startle-Reiz dargeboten (Bradley et al., 1999). Diese Vorgehensweise wurde hier ebenfalls gewählt, die Probanden erhielten sechs Startle-Reize in den Pausen zwischen den Bildern. Allerdings zeigt sich hier nicht das erwartete Ergebnis, dass die Höhe der Startle-Reaktion zwischen der auf die positiven und der auf die negativen Bilder liegt. Teilweise sind die Reaktionen sogar höher als auf die negativen Bilder. Möglicherweise ist es hier nicht wirklich gelungen, einen "neutralen" Befindlichkeitsstatus hervorzurufen, da das Warten auf neue Bilder für manche Probanden aversiv gewirkt haben könnte. Die Probanden waren angewiesen, während der Pausen (,die aus technischen Gründen zum Teil sehr lang waren, bis zu 48 Sekunden) auf ein weißes Kreuz auf dem Bildschirm zu gucken, um zu verhindern, dass sie sich im Raum umgucken. Einige Probanden äußerten spontan, dass dieses Blicken auf das Kreuz für sie sehr unangenehm gewesen sei, was die hohen Startle-Reaktionen erklären könnte. Auch wenn dies Ergebnis nicht den in der Literatur beschriebenen Ergebnissen entspricht, kann man aufgrund der Reaktionen auf die IAPS- Bildern davon ausgehen, dass die Umsetzung des Startle-Paradigmas grundsätzlich korrekt gelungen ist.

Neben der Möglichkeit, dass es mit dem gewählten Versuchsablauf nicht gelungen ist, Bewegungsangst zu aktivieren, könnte auch ein Unterschied zwischen dem Angstreiz, um den es in dieser Studie geht und den phobierelevanten Reizen der anderen Untersuchungen bedeutsam sein. In allen zitierten Studien zur Startle-Reaktion handelt es sich bei dem Angstreiz um Objekte (Spinnen, Hunde, Nahrungsmittel), während es in dieser Studie um eine Handlung geht. Welche Auswirkungen die Vorstellung von Handlungen auf die Startle-Reaktion hat, ist bisher nicht gut untersucht. Es ist nicht ganz auszuschließen, dass die Startle-Reaktion auf angstbesetzte Handlungen nicht die gleiche ist, wie die auf angstbesetzte Objekte.

Jenseits der Diskussion über mögliche methodische Gründe, weswegen die erwartete Startle-Reaktion nicht gefunden wurde, sollen auch theoretische Überlegungen angestellt werden. Es wird davon ausgegangen, dass eine Erhöhung der Startle-Reaktion die Aktivierung des defensiven motivationalen Systems der Vermeidung/ Flucht reflektiert. Dass die CLBP-Patienten bei der Betrachtung der Bewegungsbilder und der Vorstellung, die abgebildeten Bewegungen auszuführen, diese Reaktion nicht zeigen, könnte bedeuten, dass dies System hier nicht aktiviert worden ist. Die Probanden würden dann 
also keine Angst, die mit der Aktivierung dieses Systems einhergeht, erleben. Es könnte demnach sein, dass Patienten mit chronischen Rückenschmerzen keine Bewegungsangst erleben, die der Angst von Phobikern gleicht. Möglicherweise haben die Patienten stattdessen ein kognitives Konzept von der potentiellen Gefährlichkeit von Bewegungen bzw. von der Notwendigkeit, Bewegungen zu vermeiden. Dieses Schema könnte sowohl durch eigene Erfahrungen erworben sein, als auch über die Erfahrungen anderer. Diese Argumentation trägt auch Philips (1987) vor, die davon ausgeht, dass kognitive Prozesse für Vermeidungsverhalten von Schmerzpatienten entscheidender sind als das Erleben von Angst. Auf die Bedeutsamkeit kognitiver Prozesse für das Verhalten von chronischen Schmerzpatienten verweist auch Dolce (1987). Waddell et al. (1993) diskutieren, dass die Beziehung zwischen Schmerz, Bewegung und Angst unter Umständen nur in der akuten Phase der Rückenschmerzen eine Rolle spielt, während später kognitive Schemata über die Schädlichkeit von Bewegungen das Verhalten bestimmen, ohne dass Furcht aktuell erlebt wird. Da die CLBP-Patienten, die an dieser Studie teilnahmen, alle seit mehr als drei Jahren an ihren Rückenschmerzen leiden, ist es denkbar, dass eine ursprünglich konditionierte Angst ihre Bedeutung verloren hat und so durch die Konfrontation mit rückenschmerzrelevanten Bildern das System von Vermeidung/ Flucht nicht mehr aktiviert wird. Um diesen Fragen nachzugehen, wäre es interessant, eine Gruppe von Patienten mit chronifizierten Rückenschmerzen zu vergleichen mit einer Gruppe, deren Schmerzen erst kürzlich aufgetreten sind. Es ist nicht auszuschließen, dass sich bei Patienten mit akuten Rückenschmerzen eine StartleErhöhung zeigen würde, wenn sie mit rückenschmerzrelevanten Bewegungen konfrontiert würden. Sollte es tatsächlich nur zu Beginn von Rückenschmerzerkrankungen eine erlebte Bewegungsangst mit Aktivierung des defensiven motivationalen Systems geben, würde sich diese Angst dennoch von anderen phobischen Ängsten unterschieden. Patienten mit phobischer Angst vor Objekten wie Schlangen oder Spinnen behalten diese Angst über viele Jahre, selbst wenn sie nicht mit den angstrelevanten Reizen konfrontiert werden.

5.2 Untersuchung der Konzepte Bewegungsangst und Fear-Avoidance Beliefs unter Verwendung des Covariation Bias

Anders als die Ergebnisse des Startle-Experiments bestätigen die Ergebnisse zur Erhebung des Covariation Bias in Bezug auf die Experimentalgruppe die Hypothese. Wie 
angenommen zeigen die CLBP-Patienten bei den negativen Bewegungsbildern einen höheren Covariation Bias als bei den positiven Bewegungsbildern, das bedeutet, sie geben häufiger fälschlich an, dass diese Bilder zusammen mit dem aversiven Ton präsentiert wurden. Allerdings irritiert hier das Ergebnis der Kopfschmerzgruppe, denn diese Gruppe zeigt einen Covariation Bias für die positiven Bilder.

Auch für den Covariation Bias werden die Ergebnisse getrennt für Block 1 und Block 2 analysiert. Da die Probanden beim zweiten Durchgang bereits wussten, dass sie über die Assoziation der Bilder mit dem aversiven Ton befragt werden würden, sollten nur die Ergebnisse des Blocks 1 interpretiert werden. Dabei ergibt sich das gleiche Bild wie bei der Analyse der beiden Blocks gemeinsam: die CLBP-Patienten zeigen einen eindeutigen Covariation Bias in Bezug auf die negativen Bilder, die Kopfschmerzpatienten in Bezug auf die positiven Bilder. Die gesunde Kontrollgruppe zeigt keine Unterschiede zwischen den positiven und den negativen Bildern. Durch die Aufspaltung der Stichprobe in Block 1 und Block 2 wird die Stichprobengröße allerding sehr gering. Für die Kontrollgruppen gehen jeweils nur die Daten von neun Personen ein, was bei der Interpretation der Daten bedacht werden muss.

Das Ergebnis der Kopfschmerzgruppe in Bezug auf die Bewegungsbilder ist nicht plausibel zu interpretieren. Der erhöhte Covariation Bias bei den positiven Bewegungsbildern könnte die Vermutung nahe legen, dass Kopfschmerzpatienten mit Bildern von Entspannung und Ruhe eine Form von Gefahr assoziieren. Allerdings ist dann nicht nachvollziehbar, warum sie diese ebenso positiv bewerten, wie das die beiden anderen Gruppen tun. Ohne weitere Untersuchung einer größeren Stichprobe kann dieses Ergebnis nicht befriedigend erklärt werden.

Um die Validität der Untersuchung in Bezug auf den Covariation Bias überprüfen zu können, wurde auch der Covariation Bias zusätzlich zu den Bewegungsbildern bei den Bildern der IAPS erhoben. Dabei wurde untersucht, ob sich hier das gleiche Ergebnismuster ergibt wie in der Literatur berichtet. Dies ist der Fall, alle drei Gruppen zeigen einen signifikant höheren Covariation Bias für die negativen Bilder der IAPS als für die positiven. Das bedeutet, dass das Paradigma des Covariation Bias offensichtlich angemessen umgesetzt wurde.

Die Hypothese in Bezug auf den Covariation Bias der Experimentalgruppe wird, auch wenn nur der Block 1 betrachtet wird, statistisch bestätigt. Dies ist allerdings angesichts der ungewöhnlichen Daten der Kopfschmerzgruppe vorsichtig zu interpretieren. Für die CLBP-Patienten deuten, anders als bei der Kopfschmerzgruppe, die Daten der 
subjektiven Bewertung der Bilder und des Covariation Bias in die gleiche Richtung: die Patienten bewerten die rückenschmerzrelevanten Bilder negativer als die beiden anderen Gruppen und sie zeigen in Bezug auf diese Bilder einen höheren Covariation Bias als in Bezug auf die positiven Bilder.

Betrachtet man die Ergebnisse getrennt für die Subgruppen "Fear-avoidance hoch/niedrig“ zeigt sich allerdings kein Unterschied zwischen den Gruppen. Ebenso wie die Einschätzung der Valenz der Bilder ist also auch der Covariation Bias unabhängig von den Überzeugungen der CLBP-Patienten über den Zusammenhang zwischen ihren Rückenschmerzen und ihrer Arbeit bzw. Aktivitäten.

Der Covariation Bias zeigt nicht wie die Startle-Reaktion die Aktivierung des motivational/emotionalen Systems für Vermeidung/Flucht, sondern macht eher Aussagen über die Informationsverarbeitung der Person. Der entsprechende Reiz wird als bedrohlich erachtet, was aber nicht notwendigerweise heißt, dass auch auf physiologischemotionaler Ebene Angst erlebt wird. Schon Morris und Liebert (1970) haben bei ihrer Darstellung der beiden Konzepte "Worry" und "Emotionality" darauf hingewiesen, dass die Wahrnehmung und Äußerung von Angst nicht zwangsläufig mit den entsprechenden physiologischen Symptomen einhergeht. Wenn man davon ausgeht, dass die StartleReaktion eng mit physiologischen Angstreaktionen zusammen hängt, dann scheint genau dies in dieser Studie der Fall zu sein: die Rückenschmerzpatienten äußern eine negativere Einschätzung der rückenschmerzrelevanten Bilder als die anderen beiden Gruppen und gehen häufiger davon aus, dass die negativen Bilder zusammen mit dem aversiven Reiz präsentiert wurden. Das deutet darauf hin, dass sie ein kognitives Konzept haben, dass die dargestellten Bewegungen potentiell bedrohlich bzw. potentiell zu vermeiden sind. Dabei muss allerdings einschränkend betont werden, dass bei der Einschätzung der Bilder nicht danach gefragt wurde, wie bedrohlich die Bilder empfunden wurden, sondern nur, wie positiv bzw. negativ das Gefühl ist, das die Bilder hervorrufen, ist. Die Startle-Reaktion der Patienten entspricht diesen Äußerungen nicht: es gibt keinen Unterschied in der Höhe des Reflexes bei den positiven und den negativen Bildern, das heißt, das System für Vermeidung/Flucht ist nicht aktiviert . Interessant ist, dass bei keiner der drei abhängigen Variablen (Valenzeinschätzung, Startle-Reaktion und Covariation Bias) Unterschiede zu sehen sind, je nachdem ob die Patienten hohe oder niedrige Werte im FABQ zeigen. Wenn man davon ausgeht, dass die Valenzeinschätzung der Bilder und der Covariation Bias Hinweise auf ein kognitives Schema von Schädlichkeit bzw. Bedrohlichkeit bestimmter Bewegungen geben und der 
FABQ ebenfalls ein solches Schema erfassen würde, dann wäre zu erwarten gewesen, dass sich die Reaktionen der Personen, die ausgeprägtere Fear-Avoidance Beliefs äußern, von denen mit geringeren Fear-Avoidance Beliefs unterscheiden würden. Die Tatsache, dass dies hier nicht der Fall ist, weist darauf hin, dass es sich bei Fear-Avoidance Beliefs möglicherweise um andere Schemata handelt als die, die hier durch die Valenzeinschätzung und den Covariation Bias deutlich zu werden scheinen.

5.3 Untersuchung der Konzepte Bewegungsangst und Fear-Avoidance Beliefs unter Verwendung von Fragebögen

Um das Konstrukt der Fear-Avoidance Beliefs genauer zu beleuchten, wurde der FearAvoidance Beliefs Questionnaire (FABQ) mit einigen anderen Fragebögen korreliert, die Aufschluss darüber geben könnten, welche psychologischen Prozesse dieser Fragebogen erfasst. Um zu überprüfen, ob der FABQ tendenziell eher tatsächlich erlebte Angst bei Schmerzen misst oder eher generelle Einstellungen zu Verhaltensweisen in Bezug auf Schmerz, wurden zwei Fragebogen für die Erhebung dieser Prozesse erstellt, die mit dem FABQ korreliert wurden. Für die Beurteilung dieser Zusammenhänge ist es zunächst bedeutsam, ob die selbst erstellten Fragebögen zufrieden stellende Gütekriterien aufweisen. Da beide Fragebögen gute Werte für die interne Konsistenz zeigen und sich bei einer Voruntersuchung auch zufrieden stellende bis gute Werte für die RetestReliabilität ergaben, kann von einer ausreichenden Reliabilität der Fragebögen ausgegangen werden. Dass der Schmerz-Angst-Bogen (SAB) wie angestrebt eher Aspekte erlebter Angst erfasst als der Schmerz-Einstellungs-Bogen (SEB) zeigt sich daran, dass er bedeutsame Zusammenhänge zu beiden Skalen des STAI aufweist (27\% gemeinsame Varianz mit State-Angst und $38 \%$ gemeinsame Varianz mit Trait-Angst), während der SEB nur $6 \%$ gemeinsame Varianz mit Trait-Angst aufweist. Die beiden Fragebögen untereinander zeigen eine gemeinsame Varianz von nur 18\%, erfassen also im wesentlichen unterschiedliche Dimensionen. Der (schwache) Zusammenhang zwischen der Angst bei Schmerzen und der Einstellung, sich, wenn man Schmerzen hat, schonen bzw. zurückziehen zu müssen, ist plausibel.

Bei der Analyse der Zusammenhänge zwischen SAB, SEB und FABQ zeigt sich, dass der Gesamtscore des FABQ zu keinem der beiden Fragebögen einen bedeutsamen Zusammenhang aufweist. Schaut man sich die drei Faktoren des FABQ "Ursache Arbeit", "Prognose Arbeit" und "Aktivität" getrennt an, dann ergibt sich lediglich 
zwischen dem Faktor "Aktivität" und dem SEB ein auf dem 5\% Niveau signifikanter, aber eher geringer Zusammenhang von $r=-.36$. Der Faktor "Aktivität" des FABQ hat also etwas mit der generellen Einstellung zu Rückzug und Schonung gemeinsam. Die geringe Größe des Zusammenhang zwischen den Skalen lässt die Interpretation zu, dass Rückenschmerzpatienten möglicherweise nicht generell der Meinung sind, dass man sich bei Schmerzen schonen sollte (wie es die Items des SEB erfassen), sondern dass sie dies sehr spezifisch für Aktivitäten denken, von denen sie der Ansicht sind, dass sie ihrem Rücken schaden (was die FABQ-Skala erfasst). Für diese Überlegung spricht auch, dass sich bei der Betrachtung der Werte des SEB für die gesamte Stichprobe zeigt, dass sich die Rückenschmerzpatienten von der gesunden Kontrollgruppe wider Erwarten nicht unterscheiden. Das bedeutet, dass in dieser Studie die Rückenschmerzpatienten keine besonders ausgeprägte Überzeugung ausdrücken, dass man sich bei Schmerzen generell zurückziehen oder schonen sollte. Die Kopfschmerzpatienten hingegen bekunden diese Ansicht deutlich ausgeprägter als die beiden anderen Gruppen. Die Personen, die eine generelle Tendenz zu Vermeidungsverhalten bei Schmerzen äußern, sind hier also eher die Kopfschmerz- als die Rückenschmerzpatienten.

Die Zusammenhänge zwischen dem FABQ und den beiden selbst erstellten Fragebögen ergeben also aufgrund des undifferenzierten Korrelationsmuster wenig Aufschluss über die Frage, welche psychologischen Prozesse der FABQ erfasst. Da Waddell et al. (1993) die Bedeutung der Arbeitszufriedenheit im Zusammenhang mit den Fear-Avoidance Beliefs diskutiert, wurde der FABQ zusätzlich mit dem Arbeitsbeschreibungsbogen (ABB) korreliert. Tatsächlich besteht zwischen dem Faktor "Ursache Arbeit" und dem ABB der insgesamt höchste Zusammenhang der Analyse. Mit knapp 18\% gemeinsamer Varianz ist jedoch auch dieser Zusammenhang nicht besonders eng. Die beiden anderen Faktoren weisen ebenso wenig wie der Gesamtscore des FABQ bedeutsame Zusammenhänge zum $\mathrm{ABB}$ auf. Ob Personen davon ausgehen, dass ihre Arbeit ihrem Rücken schadet bzw. ihre Rückenschmerzen ausgelöst hat, scheint also in einer gewissen Verbindung damit zu stehen, wie zufrieden sie insgesamt mit ihrer Arbeit sind.

Die Faktoren "Ursache Arbeit" und "Prognose Arbeit" haben sich als sehr viel bessere Prädiktoren für Arbeitsunfähigkeit herausgestellt, als viele andere Faktoren wie Schmerzintensität, Dauer der Erkrankung oder allgemeine Ängstlichkeit (Pfingsten et al. 2000). Der hier aufscheinende Zusammenhang des Faktors "Ursache Arbeit", d.h. der Tendenz, als Ursache für seine Rückenschmerzen die eigenen Arbeit anzusehen, mit Arbeitszufriedenheit sollte deshalb noch genauer untersucht werden. Es wäre möglich, 
dass Personen, die mit ihrer Arbeit unzufrieden sind, eher dazu neigen, sich wegen ihrer Rückenschmerzen krankschreiben zu lassen. Um zu zeigen, ob generelle Unzufriedenheit mit der Arbeit einen Risikofaktor für Arbeitsunfähigkeit aufgrund von Rückenschmerzen darstellt, müssten Längsschnittuntersuchungen durchgeführt werden.

Die Analyse des Zusammenhangs zwischen dem FABQ und den beiden Skalen des STAI ergibt, dass der FABQ sowohl mit seinem Gesamtscore als auch mit dem Faktor "Ursache Arbeit" und dem Faktor "Aktivität" gemeinsame Varianz mit den beiden Skalen des STAI aufweist (zwischen 11 und 15\%). Diese Zusammenhänge sind niedriger als die mit dem SAB, dennoch ist es interessant, dass das Konzept der Fear-Avoidance Beliefs offenbar eine Verbindung sowohl zu aktueller Zustandsangst als auch zu der Trait-Komponente der Angst aufweist. Der Aspekt der Fear-Avoidance Beliefs, der mit der Überzeugung, wieder an die Arbeit zurückzukehren, verbunden ist (Faktor "Prognose Arbeit"), ist als Subfaktor sowohl von Zustandsangst als auch von Trait-Angst augenscheinlich relativ unabhängig.

Bemerkenswert ist, dass sowohl der FABQ als auch der SAB Zusammenhänge zu beiden Skalen des STAI zeigen, ihre Werte aber untereinander nicht bedeutsam korrelieren. Der FABQ zeigt also (außer beim Faktor "Prognose Arbeit") Zusammenhänge zu aktueller Zustandsangst und Eigenschaftsangst aber keinen Zusammenhang zu Angst bei Schmerzen, wie sie hier erfragt wird. Die Items des SAB fragen nicht nach Erwartungsangst im Zusammenhang von Schmerzen, also nicht nach Angst vor Schmerzen, sondern nach Angst, die beim Auftreten von Schmerzen entsteht. Möglicherweise würde der FABQ engere Zusammenhänge aufweisen zu einem Fragebogen, der nach der Angst, dass Schmerzen auftreten könnten, fragt. Zudem wird beim SAB nach Schmerzen allgemein und nicht spezifisch nach Rückenschmerzen gefragt. Ein Fragebogen, der in diesem Punkt spezifischer wäre, könnte eventuell ebenfalls stärkere Zusammenhänge zum FABQ aufweisen.

Insgesamt gibt die Analyse der Konkordanzen der angewendeten Fragebögen keinen deutlichen Aufschluss darüber, was der FABQ eigentlich misst. Schwierig ist die Interpretation auch deshalb, weil die drei Subfaktoren des FABQ offenbar doch unterschiedliche Bereiche erfassen, auch wenn sie untereinander hoch korrelieren. Der Faktor "Prognose Arbeit" zeigt zu keinem der anderen Fragebögen bedeutsame Zusammenhänge. Der deutlichste Zusammenhang ergibt sich zwischen dem Faktor "Ursache Arbeit", also der Neigung, Aspekte der Arbeit als Ursache für die Rückenschmerzen anzunehmen, und dem ABB. Eine weitere, wenn auch geringere 
Korrelation zeigt sich zwischen dem Faktor "Aktivität" und dem SEB. So ergibt die Analyse, auch wenn die Ergebnisse keine eindeutigen Interpretationen zulassen, doch Hinweise darauf, dass das Konzept der Fear-Avoidance Beliefs eher Arbeitszufriedenheit bzw. kognitive Schemata über die Schädlichkeit bestimmter Aktivitäten bzw. der Arbeit für den Rücken widerspiegelt als tatsächlich erlebte Angst im Zusammenhang mit Schmerzen. Um mehr Aufschluss über das Konzept zu bekommen, sollten größere Stichproben untersucht werden. Bei weiteren Untersuchungen sollte das Konzept der Angst im Zusammenhang mit Schmerzen jedoch noch deutlicher differenziert werden. Zudem wäre es sicher aufschlussreich, die Prozesse, die die beiden selbst erstellten Fragebögen hier erfassen sollten, zusätzlich spezifisch für Rückenschmerzen zu formulieren und eventuelle Unterschiede zu den unspezifisch formulierten Konzepten zu erfassen.

\subsection{Gesamtdiskussion}

Die Ausgangsfragestellung dieser Studie war die, ob Patienten mit chronischen Rückenschmerzen tatsächlich Angst vor Bewegung erleben oder ob es sich bei dieser so genannten Kinesiophobie eher um überdauernde Überzeugungen über die Schädlichkeit bestimmter Bewegungen handelt, die nicht zwangsläufig mit dem Erleben von Angst in Verbindung stehen. Die Ergebnisse dieser Studie deuten darauf hin, dass Rückenschmerzpatienten bestimmte Bewegungen negativ bewerten und ein Konzept der potentiellen Gefährlichkeit dieser Bewegung ausgebildet haben, worauf der Covariation Bias hinweist. Dass Patienten bei der Konfrontation mit solchen Bewegungen auch tatsächlich Angst erleben, konnte in dieser Studie nicht gezeigt werden. Möglicherweise haben die Patienten ein kognitives Konzept der Schädlichkeit bestimmter Bewegungen, das aber nicht mit dem Erleben von Angst in Verbindung steht. Was in dieser Studie nicht untersucht wurde, ist, ob Rückenschmerzpatienten die von ihnen als schädlich oder gefährlich eingestuften Bewegungen auch tatsächlich vermeiden würden. Nach allen bisherigen Untersuchungen, die im ersten Teil dieser Arbeit referiert wurden, ist dies durchaus wahrscheinlich. Vermeidungsverhalten kann jedoch auch unabhängig von dem Erleben von Angst gezeigt werden, wie bereits vielfach in Tierexperimenten und klinischen Studien nachgewiesen wurde (Mineka und Gino, 1980, Rachman und Hodgson, 1974). Rachman (2000) betont sogar, dass Furcht nicht notwendig ist, um überhaupt Vermeidungsverhalten herauszubilden. Ebenso könne Modelllernen zu 
Vermeidungsverhalten führen oder das Übernehmen von Konzepten von Gefährlichkeit von signifikanten Anderen. Es ist also möglich, und die Befunde der Studie deuten in die Richtung, dass Rückenschmerzpatienten ein Konzept der Schädlichkeit/Gefährlichkeit von bestimmten Bewegungen haben, ohne jemals selbst Angst vor diesen Bewegungen zu erleben. Das würde bedeuten, dass das Konzept der Gefährlichkeit von Bewegungen, das $\mathrm{zu}$ vermehrter Vermeidung und nachfolgend $\mathrm{zu}$ Beeinträchtigungen führt, nicht zwangsläufig durch klassische Konditionierung im akuten Stadium der Schmerzen erworben sein muss. Vielmehr wäre die Frage, welche anderen Faktoren zur Ausbildung solcher Konzepte beitragen. Hier wäre es sicherlich interessant, Überzeugungen von Familienmitglieder zu erheben, um den Zusammenhang mit den Konzepten der Patienten $\mathrm{zu}$ überprüfen. Ebenso bedeutsam ist es sicherlich, welche Anweisungen und Empfehlungen Patienten in diesem Zusammenhang von ihren Ärzten erhalten. So ist es denkbar, dass Ratschläge wie der, sich mit einem akuten Rückenschmerz zwei Wochen ins Bett zu legen und möglichst wenig zu tun, zu einer Ausbildung eines Konzepts von "Bewegungsangst" beitragen. Es ist aber auch nicht auszuschließen, dass es bei verschiedenen Patienten verschiedene Wege zur Ausbildung von "Bewegungsangst" gibt. So mögen bestimmte Patienten tatsächlich erlebt haben, dass bestimmte Bewegungen extrem schmerzhaft sind und dass Ruhe und Schonung die Schmerzen verringern. Andere mögen sich eher an die Aussagen ihrer Ärzte halten oder auch an Richtlinien von Rückenschulen, die bestimmte Bewegungen regelrecht zum Tabu erklären. Entscheidend ist auf jeden Fall die Vermeidungstendenz, um deren negative Folgen es letztendlich geht. Natürlich ist für die Frage, wie die Neigung zur Vermeidung verändert werden kann, wieder die Frage bedeutsam, vor welchem Hintergrund diese Vermeidung überhaupt stattfindet. Sind es tatsächlich eigene Erfahrungen, die die Patienten Bewegungen vermeiden lassen, so ist es in der Therapie notwendig, den Patienten die Möglichkeit zu geben, neue Erfahrungen zu machen und auf lange Sicht zu lernen, dass Bewegungen nicht immer schädlich sind. Sind es mehr von anderen übernommene Konzepte, die die Patienten dazu bringen, Bewegungen zu vermeiden, so muss die Therapie auf die Veränderung solcher Konzepte ausgerichtet sein. Es wird dann wenig nützen, wenn der Patient zum Beispiel in der Krankengymnastik erfährt, dass bestimmte Bewegungen ihm eher Nutzen als Schaden bringen, wenn weiterhin von anderen Personen wie Ärzten oder auch Familienangehörigen vertreten wird, dass ein Mensch mit chronischen Rückenschmerzen Bewegungen möglichst vermeiden sollte. Multimodale Therapiekonzepte wie das Göttinger Rücken Intensiv Programm sind in 
diesem Zusammenhang besonders nützlich, da sie beiden Aspekten von Bewegungsangst Rechnung tragen und sowohl eine Veränderung negativer Erfahrungen als auch eine kognitive Umstrukturierung ungünstiger Überzeugungen erlauben (Pfingsten, Hildebrandt, Franz und Saur, 1997).

Die vorliegende Studie geht der Frage nach, ob Fear-Avoidance Beliefs, die sich als Prädiktor für Beeinträchtigung und Arbeitsunfähigkeit bewährt haben, in Zusammenhang mit Bewegungsangst stehen, oder ob die Konzepte eher unabhängig voneinander zu betrachten sind. Die Ergebnisse in Bezug auf die Startle-Reaktion, die ValenzEinschätzung und den Covariation Bias werden jeweils nicht durch das Ausmaß der Fear-Avoidance-Beliefs beeinflusst. Interessant sind dabei die Ergebnisse zur Valenzeinschätzung und zum Covariation Bias. In beiden Fällen zeigen die CLBPPatienten Unterschiede zu den beiden anderen Gruppen, die darauf hindeuten, dass sie die Bewegungen negativer empfinden und als gefährlicher einstufen als die andern Probanden. Die CLBP-Patienten scheinen also spezifisch ein Konzept von Gefährlichkeit von Bewegungen auszubilden. Da es dabei keine Unterschiede gibt, je nachdem ob die Patienten ausgeprägte oder weniger ausgeprägte Fear-Avoidance Beliefs aufweisen, scheinen Fear-Avoidance Beliefs noch etwas anderes zu beeinhalten als das Konzept, dass bestimmte Bewegungen gefährlich sind. Leider geben die Analysen der Zusammenhänge zu anderen Fragebögen nicht besonders viel Aufschluss über diese Frage. Auffallend ist, dass beim Fear-Avoidance Beliefs Questionnaire in vielen Items der Begriff des Schadens vorkommt (z.B. "Körperliche Aktivitäten könnten meinem Rücken schaden“). Vlaeyen et al. (1995) sprechen neben einer Angst vor Bewegungen auch von einer Angst vor Verletzung bzw. Schaden ("Fear of (re)injury“). Sie gehen davon aus, dass Patienten insbesondere dann Bewegungen vermeiden, wenn sie der Ansicht sind, dass die Bewegungen dem Rücken schaden und nicht nur, wenn sie durch diese Bewegungen Schmerz erwarten. Es wäre möglich, dass die Valenzeinschätzung der rückenschmerzrelevanten Bilder und der Covariation Bias ein kognitives Konzept widerspiegeln, dass Bewegungen unangenehm und tendenziell gefährlich sind, weil sie schmerzhaft sein könnten. Möglicherweise gehen aber nicht alle Patienten auch davon aus, dass diese Bewegungen dem Rücken schaden. Wenn der Fear-Avoidance Beliefs Questionnaire tatsächlich eher diese Erwartung von Schaden misst, dann könnte das erklären, warum es keine Unterschiede bei der Valenzeinschätzung bzw. beim Covariation Bias gibt, je nachdem, ob die Patienten ausgeprägte Fear-Avoidance Beliefs aufweisen oder nicht. Das Fear-Avoidance-Konzept würde dann also eher Erwartung von 
Schaden erfassen, während die Valenzeinschätzung und der Covariation Bias eher ein kognitives Konzept von Bewegungsangst widerspiegeln, ein kognitives Schema, dass Bewegungen Schmerzen hervorrufen könnten und deshalb vermieden werden sollten. Interessant wäre in diesem Zusammenhang natürlich die Frage, ob Menschen mit beiden Konzepten eher vermeiden und stärker beeinträchtigt sind, als Menschen, die nicht meinen, dass bestimmte Bewegungen ihrem Rücken schaden bzw. Schmerzen hervorrufen. Da, wie bereits betont, Vermeidungsverhalten in dieser Studie nicht erhoben wurde, kann darüber mit den vorliegenden Daten keine Aussage gemacht werden.

Bei der Fragebogenuntersuchung zeigt sich als besonders interessantes Ergebnis, dass die Rückenschmerzpatienten sich in ihrer generellen Tendenz zu Vermeidung und Rückzug nicht von den gesunden Probanden unterscheiden. Anders als die Kopfschmerzpatienten dieser Studie zeigen sie nicht stärker als gesunde Personen eine Neigung, sich bei Schmerzen zurückzuziehen und zu schonen. Das deutet darauf hin, dass es tatsächlich sinnvoll ist, diese beiden Schmerzgruppen getrennt voneinander zu behandeln und bei Rückenschmerzpatienten auf ganz spezifische Aspekte einzugehen, die Vermeidungsverhalten bei ihnen hervorrufen.

Wenn man einmal davon ausgeht, dass die Ergebnisse dieser Studie nicht durch methodische Mängel beeinflusst worden sind, dann lässt sich also festhalten, dass bei Patienten mit chronischen Rückenschmerzen in dieser Untersuchung keine Anzeichen dafür bestehen, dass sie aktuell Angst vor Bewegung erleben. Ebenso deuten die Ergebnisse darauf hin, dass es ein kognitives Konzept der Gefährlichkeit von bestimmten Bewegungen bei diesen Patienten gibt (d.h. Bewegungen werden als gefährlich angesehen, weil sie Schmerzen hervorrufen können), dass dies Konzept aber möglicherweise unabhängig von einem Konzept von Schädlichkeit im Sinne drohender Verletzung des Rückens ist.

Da die Frage nach methodischen Mängeln hier jedoch nicht eindeutig beantwortet werden kann, muss diese Interpretation der vorliegenden Daten mit Vorsicht vorgenommen werden. Nicht zuletzt ist noch einmal zu betonen, dass die Stichprobengröße bei einigen Analysen nicht ausreicht, um fundierte Aussagen machen zu können. Generell hat sich in dieser Studie gezeigt, dass es sinnvoll ist, die Ursache von Vermeidungsverhalten bei Rückenschmerzpatienten sehr spezifisch zu untersuchen. Da die Frage, ob ungünstige Überzeugungen über die Gefährlichkeit bzw. Schädlichkeit bestimmter Bewegungen durch eigene Erfahrungen erworben sind oder eher durch das Übernehmen von Überzeugungen anderer Personen, für die Therapie bedeutsam 
erscheint, sollten zu diesem Punkt weitere Untersuchungen erfolgen. Diese Frage ist auch jenseits von Überlegungen für die Therapie insofern interessant, als sie für die Ausbildung von Personen, die Patienten mit chronischen Rückenschmerzen behandeln, entscheidend sein könnte. Sollte sich zeigen, dass diese Konzepte tatsächlich stark durch das Verhalten von Ärzten bzw. Krankengymnasten oder anderem medizinischen Personal geprägt werden, so sollten diese Personen für diese Effekte sensibilisiert werden. Zudem wäre es wichtig zu untersuchen, wie die Konzepte dieser Personen in Bezug auf Bewegungen bei Rückenschmerzpatienten aussehen, und ob sich diese möglicherweise gewandelt haben. Sollte sich herausstellen, dass bei dem mit Rückenschmerzpatienten befassten medizinischem Personal tatsächlich Einstellungen vorherrschen, dass Bewegungen für Rückenschmerzpatienten generell eher als schädlich anzusehen sind und deshalb Vermeidungsverhalten anzuraten ist, erscheint es dringend geboten, in Aus- und Weiterbildung diese Einstellungen zu verändern. Gerade vor dem Hintergrund der Beobachtung, dass die Beeinträchtigung durch Rückenschmerzen offensichtlich in den letzten Jahren dramatisch zugenommen hat, erscheint die weitere Untersuchung der hier aufgeworfenen Fragen außerordentlich bedeutsam. 


\section{Literatur}

Allan, D. B., \& Waddell, G. (1989). An historical perspective on low back pain and disability. Acta Orthopaedica Scandinavia, 60 (Supplement 234), 1-23.

Amin, J. M., \& Lovibond, P. F. (1997). Dissociations between covariation bias and expectancy bias for fear-relevant stimuli. Cognition and Emotion, 11(3), 273-289.

Angrilli, A., Mauri, A., Palomba, D., Flor, H., Birbaumer, N., Sartori, G., \& di Paola, F. (1996). Startle relflex and emotion modulation impairment after a right amygdala lesion. Brain, 119, 1991-2000.

Anthony, B. J., \& Graham, F. K. (1985). Blink reflex modification by selective attention: evidence for the modulation of 'automatic' processing. Biological Psychology, 21, 43-59.

Asmundson, G. J. G., Kuperos, J. L., \& Norton, G. R. (1997). Do patients with chronic pain selectively attend to pain-related information?: Preliminary evidence for the mediating role of fear. pain, 72, 27 - 32 .

Asmundson, G. J. G., \& Norton, R. (1995). Anxiety sensitivity in patients with physically unexplained chronic back pain: A preliminary report. Behavior Research and Therapy, 33, 771-777.

Asmundson, J. G., \& Taylor, S. (1996). Role of Anxiety Sensitivity in Pain-Related Fear and Avoidance. Journal of Behavioral Medicine, 19, 577-587.

Berg, K., \& Balaban, M. T. (1999). Startle elicitation: Stimulus parameters, recording techniques, and quantification. In M. E. Dawson \& A. M. Schell \& A. H. Boehmelt (Eds.), Startle modification. Cambridge: Cambridge University Press.

Bigos, S. J., Battie, M. C., Spengler, D. M., Fisher, L. D., Fordyce, W. E., Hansson, T. H., Nachemson, A. L., \& Wortley, M. D. (1991). A prospective study of work perceptions and psychosocial factors affecting the report of back injury. Spine, 16, $1-6$.

Blanley, P. H. (1986). Affect and memory: a review. Psychological Bulletin, 99, 229246.

Bortz, J., \& Döring, N. (1995). Forschungsmethoden und Evaluation . Berlin: SpringerVerlag.

Bortz, J., Lienert, G. A., \& Boehnke, K. (1990). Verteilungsfreie Methoden in der Biostatistik. Berlin: Springer Verlag. 
Bradley, M. M., Cuthbert, B. N., \& Lang, P. J. (1990). Startle reflex modification: Emotion or attention? Psychophysiology, 27(5), 513-523.

Bradley, M. M., Cuthbert, B. N., \& Lang, P. J. (1999). Affective Modification of Startle. In M. E. Dawson \& A. M. Schell \& A. H. Boehmelt (Eds.), Startle Modification (pp. 157-183). Cambridge: Cambridge University Press.

Bradley, M. M., \& Lang, P. J. (1994). Measuring emotion: the self-assessment manikin and the semantic differential. Journal of Behavior Therapy and Experimental Psychiatry, 25, 49-59.

Breuer, P. (1998). Steuerprogramm für den Macintosh.Unpublished manuscript.

Büssing, A. (1991). Struktur und Dynamik von Arbeitszufriedenheit. Konzeptuelle und methodische Überlegungen zu einer Untersuchung verschiedener Formen von Arbeitszufriedenheit. In L. Fischer (Ed.), Arbeitszufriedenheit (Vol. 5, pp. 1-20). Stuttgart: Verlag für Angewandte Psychologie.

Cook, E. W., Hawk, L. W., Davis, T. L., \& Stevenson, V. E. (1991). Affective indivual differences and startle reflex modulation. Journal of Abnormal Psychology, $100(1), 5-13$.

Cox, B. J., Parker, J. D. A., \& Swinson, R. P. (1996). Anxiety sensitivity: Confirmatory evidence for a multidimensional construct. Behavior Research and Therapy, 34(7), 591-598.

Crombez, G., Eccleston, C., Baeyens, F., \& Eelen, P. (1996). The disruptive nature of pain: an experimental investigation. Behavior Research and Therapy, 34(11/12), $911-918$.

Crombez, G., Vlaeyen, J. W. S., Heuts, P. H. T. G., \& Lysens, R. (1999). Pain-related fear is more disabling than pain itself: evidence on the role of pain-related fear in chronic back pain disability. Pain, 80, 329-339.

Cuthbert, B. N., Bradley, M. M., \& Lang, P. J. (1996). Probing picture perception: Activation and emotion. Psychophysiology, 33, 103-111.

Davey, G. C. L., \& Dixon, A. L. (1996). The expectancy bias model of selective associations: the relationship of judgements of cs dangerousness, CS-UCS similarity and prior fear to a priori and a posteriori covariation assessments. Behavior Research and Therapy, 34(3), 235-252.

Davis, M., Walker, D. L., \& Lee, Y. (1999). Neurophysiology and Neuropharmacology of Startle and Its Affective Modulation. In M. E. Dawson \& A. M. Schell \& A. H. Boehmelt (Eds.), Startle modification. Cambridge: Cambridge University Press. 
DeJong, P. J., \& Merckelbach, H. (1991). Covariation bias and electrodermal responding in spider phobics before and after Behavioral treatment. Behavior Research and Therapy, 29, 307-314.

DeJong, P. J., Merckelbach, H., \& Arntz, A. (1991). Eyeblink startle responses in spider phobics before and after treatment: A pilot study. Behavioral Assessment, 13, 213-223.

Dolce, J. J. (1987). Self efficacy and disability beliefs in behavioral treatment of pain. Behavior Research and Therapy, 25, 289-299.

Filion, D. L., Dawson, M. E., \& Schell, A. M. (1998). The psychological significance of human startle eyeblink modification: a review. Biological Psychology, 47, 1-43.

Flor, H., Breitenstein, C., Birbaumer, N., \& Fürst, M. (1995). A psychophysiological analysis of operant reinforcement, spouse interaction, and pain perception. Behavior Therapy, 26, 255-272.

Flor, H., Kerns, R. D., \& Turk, D. C. (1986). The role of spouse reinforcement, perceived pain activity levels in chronic pain patients. Journal of Psychosomatic Research, 31, 251-259.

Flor, H., Turk, D. C., \& Scholz, O. B. (1987). Impact of chronic pain on the spouse: Marital, emotional, and physical consequences. Journal of Psychosomatic Research, 31, 63-71.

Fordyce, W. E. (1976). Behavioral methods for chronic pain and illness. St Louis: Moseby.

Fordyce, W. E. e. (1995). Back pain in the workplace. Seattle: IASP Press.

Grillon, C., Rezvan, A., Woods, S. W., Merikangas, K., \& Davis, M. (1991). Fearpotentiated startle in humans: Effects of anticipatory anxiety on the acoustic blink reflex. Psychophysiology, 28(5), 588-595.

Hamm, A., Cuthbert, B. N., Globisch, J., \& Vaitl, D. (1997). Fear and the startle reflex: Blink modulation and autonomic response patterns in animal and mutilation fearful subjects. Psychophysiology, 34, 97-107.

Hamm, A., Gerlach, M., Globisch, J., \& Vaitl, D. (1992). Phobia specific startle reflex modulation during affective imagery and slide viewing. Psychophysiology, 29, S36.

Hamm, A. O., \& Vaitl, D. (1996). Affective learning: Awareness and aversion. Psychophysiology, 33, 698-710. 
Harper, A. C., Harper, D. A., Lambert, L. J., Andrews, H. B., Lo, S. K., Ross, F. M., \& Straker, L. M. (1992). Symptoms of impairment, disability and handicap in low back pain: a taxonomy. Pain, 50, 189.

Hasenbring, M. (1999). Prozesse der Chronifizierung von Schmerzen. In H. D. Basler \& C. Franz \& B. Kroener-Herwig \& H. P. Rehfisch \& H. Seemann (Eds.), Psychologische Schmerztherapie. Berlin: Springer.

Jäckel, W. H., \& Gerdes, N. (1998). Medizinische Rehabilitation bei Rückenschmerzen die Situation in Deutschland. In M. Pfingsten \& J. Hildebrandt (Eds.), Chronischer Rückenschmerz. Wege aus dem Dilemma. Bern; Göttingen; Toronto; Seattle: Huber.

Klenerman, L., Slade, P. D., Stanley, M., Pennie, B., Reilly, J. P., Atchison, L. E., Troup, J. D. G., \& Rose, M. J. (1995). The Prediction of Chronicity in Patients With an Acute Attack of Low Back Pain in a General Practice Setting. Spine, 20(4), 478 484.

Kori, S. H., P., M. R., \& Todd, D. D. (1990). Kinesiophobia: A new view of chronic pain behavior. Pain Mangement, Jan/Feb, 35-43.

Kröner-Herwig, B. (1999). Chronischer Schmerz - Eine Gegenstandsbestimmung. In H. D. Basler \& C. Franz \& B. Kroener-Herwig \& H. P. Rehfisch \& H. Seemann (Eds.), Psychologische Schmerztherapie. Berlin: Springer.

Kröner-Herwig, B . (2000). Rückenschmerz. Göttingen, Bern, Toronto, Seattle: Hogrefe. Kröner-Herwig, B., Jäkle, C., Frettlöh, J., Peters, K., Seemann, H., Franz, C., \& Basler, H.-D. (1996). Predicting Subjective Disability in Chronic Pain Patients. International Journal of Behavioral Medicine, 3(1), 30 - 41.

Lang, P. J., Bradley, M. M., \& Cuthbert, B. N. (1990). Emotion, attention, and the startle reflex. Psychophysiological Review, 97, 377-395.

Lang, P. J., Bradley, M. M., \& Cuthbert, B. N. (1997). International Affective Picture System: NMH Center for the Study of Emotion and Attention.

Lang, P. J., Greenwald, M. K., Bradley, M. M., \& Hamm, A. O. (1993). Looking at pictures: Affective, facial, visceral and behavioral reactions. Psychophysiology, 30, 261-273.

Laux, L., Glanzmann, P., Schaffner, P., \& Spielberger, C. D. (1981). Das State-Trait Angstinventar (STAI). Weinheim: Beltz Test Gesellschaft. 
Lethem, J., Slade, P. D., Troup, J. D. G., \& Bentley, G. (1983). Outline of a fearavoidance model of exaggerated pain percepiton. Behavior Research and Therapy, 21(4), 401-408.

Liebert, R. M., \& Morris, L. W. (1967). Cognitive and emotional components of test anxiety: A distinction and some initial data. Psychological Reports, 29, 975-978.

Linton, S. J., \& Warg, L.-E. (1993). Attributions (beliefs) and job satisfaction associated with back pain in an industrial setting. Perceptual-and-Motor-Skills, 76(1), 51-62.

Lousberg, R., Schmidt, A. J., \& Groenman, N. H. (1992). The relationship between spouse solicitousness and pain behavior: searching for more experimental evidence. Pain, 51, 75-79.

Mauler, B., Tuschen, B., \& Hamm, A. (1997). Fear or craving: Startle reflex modulation during viewing of food cues in deprived and nondeprived bulimics. Psychophysiology, 34(S1), 17.

McCracken, L., Gross, R. T., Aikens, J., \& Carnike Jr., C. L. M. (1996). The assessment of anxiety and fear in persons with chronic pain: a comparison of instruments. Behavior Research and Therapy, 34, 927-933.

McCracken, L., Gross, T., Sorg, P. J., \& Edmands, T. A. (1993). Prediction of pain in patients with chronic low back pain: effects of inaccurate prediction and painrelated anxiety. Behavior Research and Therapy, 31, 647-652.

McCracken, L., Zayfert, C., \& Gross, R. T. (1992). The Pain anxiety Symptoms Scale: development and validation of a scale to measure fear of pain. Pain 50, 67-73.

Melzak, R., \& Wall, P. (1982). The Challenge of Pain. London: Penguin Books.

Miller, R. P., Kori, S. H., \& Todd, D. D. (1991). The Tampa Scale.Unpublished manuscript, Tampa FL.

Mineka, S., \& Gino, A. (1980). Dissociation between conditioned emotional response and extended avoidance performance. Learning and motivation, 11, 416-502.

Morris, L. W., \& Liebert, R. M. (1970). Relationship of cognitive and emotional components of test anxiety to physiological arousal and academic performance. Journal of Consulting and Clinical Psychology, 35, 332-337.

Mowrer, O. H. (1939). Stimulus response theory of anxiety. Psychological Review, 46, 553-565.

Mummendey, H.-D. (1995). Die Fragebogen-Methode: Grundlagen und Anwendung in Persönlichkeits-, Einstellungs- und Selbstkonzeptforschung. Göttingen;Bern; Toronto; Seattle: Hogrefe. 
Neuberger, O., \& Allerbeck, M. (1978). Messung und Analyse von Arbeitszufriedenheit. Erfahrungen mit dem Arbeitszufriedenheitsfragebogen. Bern: Huber.

Papageorgiou, A. C., Croft, P. R., Ferry, S., Jayson, M. I. V., \& Silman, A. J. (1995).

Estimating the prevalence of low back pain in the general population. Evidence

from the South Manchester back pain survey. Spine, 20,1889-1894.

Papageorgiou, A. C., Macfarlane, G. F., Thomas, E., Croft, P. R., Jayson, M. I. V., \&

Silman, A. J. (1997). Psychosocial factors in the workplace - do they predict new episodes of low back pain? Spine, 22, 1137-1142.

Pauli, P., Montaya, P., \& Martz, G.-E. (1996). Covariation Bias in Panic-Prone

Individuals. Journal of Abnormal Psychology, 105(4), 658-662.

Pennebaker, J. W. (1982). The psychology of physical symptoms.: Springer.

Pfingsten, M., Hildebrandt, J., Franz, C., \& Saur, P. (1997). Das Göttinger Rücken

Intensiv Programm (GRIP), Teil 4: Prognostik und Fazit. Der Schmerz, 11, 30-41.

Pfingsten, M., Kröner-Herwig, B., Hempel, D., Harter, W., \& Hildebrandt, J. (1999).

Fear-avoidance Verhalten in Abhängigkeit von der Erwartungsinduktion. Der Schmerz, 13, S81.

Pfingsten, M., Kröner-Herwig, B., Leibing, E., Kronshage, U., \& Hildebrandt, J. (2000).

Validation of the German version of the Fear-Avoidance-Beliefs Questionnaire

(FABQ). European Journal of Pain, 4, 259-266.

Philips, H. C. (1987). Avoidance Behavior and its role in sustaining chronic pain.

Behavior Research and Therapy, 25, 273-279.

Putnam, L. E., \& Vanman, E. J. (1999). Long lead interval startle modification. In M. E.

Dawson \& A. M. Schell \& A. H. Boehmelt (Eds.), Startle Modification.

Cambridge: Cambridge University Press.

Rachman, S. J. (2000). Angst. Diagnose, Klassifikation und Therapie. Göttingen: Huber.

Rachman, S. J., \& Hodgson, R. (1974). Synchrony and desynchrony in fear and avoidance. Behavior Research and Therapy, 12, 311-318.

Raspe, H., \& Kohlmann, T. (1998). Die aktuelle Rückenschmerz-Epidemie. In M.

Pfingsten \& J. Hildebrandt (Eds.), Chronischer Rückenschmerz (pp. 20-36). Bern; Göttingen; Toronto; Seattle: Huber.

Raspe, T., \& Kohlmann, H. (1994). Die patientennahe Diagnostik von

Funktionseinschränkungen im Alltag. Psychomed, 6, 21-27. 
Reiss, S., Peterson, R. A., \& Gursky, D. M. (1988). Anxiety sensitivity, injury sensitivity, and individual differences in fearfulness. Behavior Research and Therapy, 26(4), 341-345.

Reiss, S., Peterson, R. A., Gursky, D. M., \& McNally, R. J. (1986). Anxiety sensitivity, anxiety frequency and the prediction of fearfulness. Behavior Research and Therapy, 24(1), 1-8.

Schiefele, U. (1990). Einstellung, Selbstkonsistenz und Verhalten. Göttingen, Toronto, Zürich: Verlag für Psychologie, Dr. C.J. Hogrefe.

Slater, M. A., Hall, H. F., Atkinson, J. H., \& Garfin, S. R. (1991). Pain and impairment beliefs in chronic low back pain: validation of the Pain and Impairment Relationship Scale (PAIRS). Pain, 48, 227-236.

Tomarken, A. J., Mineka, S., \& Cook, M. (1989). Fear-relevant selective associations and covariation bias. Journal of Abnormal Psychology, 98, 381-194.

van der Giezen, A. M., Bouter, L. M., \& Nijhuis, F. J. N. (2000). Prediction of return-towork of low back pain patients sicklisted for 3-4 month. Pain, 87, 285-294.

van Doorn, J. W. (1998). Kann eine frühzeitige Behandlung von Rückenschmerzen Arbeitsunfähigkeit sowie Chronifizierung verhindern und Kosten sparen? In M. Pfingsten \& J. Hildebrandt (Eds.), Chronischer Rückenschmerz. Bern: Verlag Hans Huber.

van Poppel, M. N. M., Koes, B. W., Deville, W., Smid, T., \& Bouter, L. M. (1998). Risk factors for back pain incidence in industry: A prospective study. Pain, 77(1), 8186.

Vanman, E. J., Boehmelt, A. H., Dawson, M. E., \& Schell, A. M. (1996). The varying time courses of attentional and affecitve modulation of the startle eyeblink reflex. Psychophysiology, 33, 691-698.

Varty, G. B., Hauger, R. L., \& Geyer, M. A. (1998). Aging effects on the startle response and startle plasticity in Fischer F344 rats. Neurobiology of Aging, 19(3), 243-251.

Vlaeyen, G., Kole-Snijders, A. M. J., Boeren, R. G. B., \& van Eek, H. (1995). Fear of movement/(re)injury in chronic low back pain and its relation to behavioral performance. Pain, 62, 363-372.

Vlaeyen, J. W. S., \& Linton, S. J. (2000). Fear-avoidance and its consequences in chronic musculoskeletal pain: a state of the art. Pain, 85(3), 317-332. 
Vlaeyen, J. W. S., Seelen, H. A. M., Peters, M., de Jong, P., Aretz, E., Beisiegel, E., \& Weber, W. E. J. (1999). Fear of movement/reinjury and muscular reactivity in chronic low back pain patients: an experimental investigation. Pain, 82(3), 297304.

Vrana, S.R., Spence, E. L., \& Lang, P. J. (1988). The startle probe response: An new measure of emotion? Journal of Abnormal Psychology, 97(4), 487-491.

Vrana, S. R., \& Lang, P. J. (1990). Fear imagery and the startle-probe reflex. Journal of Abnormal Psychology, 99(2), 189-197.

Waddell, G. (1996). Keynote address for primary care forum. Low back pain: a twentieth century health care enigma. Spine, 21(24), 2820-2825.

Waddell, G. (1998). The Back Pain Revolution. Edingburgh London New York Philadelphia Sydney Toronto: Churchill Livingstone.

Waddell, G., Newton, M., Henderson, I., Sommerville, D., \& Main, C. J. (1993). A fearavoidance beliefs questionnaire (FABQ) and the role fo fear-avoidance beliefs in chronic low back pain. Pain, 52, 157-168.

Waddell, G., Sommerville, D., Henderson, I., \& Newton, M. (1992). Objective clinical evaluation of physical impairment in chronic low back pain. Spine, 17, 617-628.

Walsh, K., Cruddas, M., \& Coggon, D. (1992). Low back pain in eigth areas of Britain. Journal of Epidemiology and Community Health, 46, 227-230. 


\section{ANHANG}

Anhang A

Anhang B

Anhang C
Informationen und Instruktionen

$\begin{array}{ll}\text { A1 } & \begin{array}{l}\text { Informationen für CLBP-Patienten vor } \\ \text { Teilnahme }\end{array} \\ \text { A2 } & \begin{array}{l}\text { Informationen für Teilnehmer und } \\ \text { Einverständniserklärung }\end{array} \\ \text { A3 } & \begin{array}{l}\text { Personenangaben } \\ \text { A4 }\end{array} \\ \text { Zusatzinformationen von Kopfschmerzpatienten } \\ \text { A5 } & \text { Instruktion 1. Teil } \\ \text { A6 } & \text { Instruktion 2. Teil } \\ \text { A7 } & \text { Instruktion 3. Teil } \\ \text { A8 } & \text { Instruktion 4. Teil } \\ \text { A9 } & \text { Instruktion 5. Teil }\end{array}$

Bilder und Fragebögen

$\begin{array}{ll}\text { B1 } & \text { Bilder Bewegung }: ; \\ \text { B2 } & \text { Bilder Bewegung }: \\ \text { B3 } & \text { Bilder IAPS :-; } \\ \text { B4 } & \text { Bilder IAPS }: \\ \text { B5 } & \text { Bilderreihenfolgen Bewegungsbilder } \\ \text { B6 } & \text { Bilderreihenfolgen IAPS-Bilder } \\ \text { B7 } & \text { Bilderevaluationsbogen } \\ \text { B8 } & \text { FABQ } \\ \text { B9 } & \text { SAB } \\ \text { B10 } & \text { SEB } \\ \text { B11 } & \text { ABB } \\ \text { B12 } & \text { STAI-State } \\ \text { B13 } & \text { STAI-Trait }\end{array}$

Ergebnisse

C1 Gruppenunterschiede Experimentalgruppe, Stichprobe aus Klinikum

C2 Startle-Reaktion in Differenzwerten und auf Blanks

C3 Covariation Bias Block 2

C4 Faktorenanalyse SAB

C5 Faktorenanalyse SEB

C6 Interkorrelationen FABQ mit Subskalen 


\section{Teilnahme an einer Untersuchung zu \\ chronischen Schmerzen}

Sehr geehrte Patientin, sehr geehrter Patient,

Sie haben sich mit Ihren Schmerzbeschwerden an die Göttinger Schmerzambulanz gewandt. Im Rahmen der dort stattfindenden Diagnostik möchten wir Sie bitten, an einer Untersuchung teilzunehmen. Dort wird die Frage untersucht, wie Patienten mit Schmerzen auf verschiedenes Bildmaterial körperlich reagieren. Die Untersuchung soll prüfen, ob es Unterschiede zwischen Patienten mit unterschiedlichen Arten von chronischen Schmerzen gibt. Möglicherweise lassen sich daraus Hinweise auf bestimmte Behandlungsformen ableiten.

Bei der Untersuchung werden die Reaktionen Ihres Augenmuskels gemessen, während Sie verschiedene Bilder auf einem Bildschirm betrachten. Dafür werden kleine Meßfühler unterhalb Ihres Auges angebracht, die die Aktivität des Muskels, der für den Lidschlag zuständig ist, erfassen können.

Die Untersuchung findet im Psychologischen Institut, Goßlerstraße 14, statt.

Sie wird ungefähr eine bis eineinhalb Stunden dauern. Wir bitten Sie, mit Herrn Dr. Pfingsten einen Termin auszumachen. Wenn möglich wird der Termin so gelegt, daß er vor oder nach einem der Untersuchungstermine im Klinikum liegt. Etwaige Kosten für die Anfahrt bzw. Parkgebühren werden Ihnen zurückerstattet.

Wenn Sie zum Lesen eine Brille benötigen, bringen Sie diese bitte mit.

Sie können sich auch direkt an Frau Dipl.-Psych.Kronshage wenden, Telefon 393592 , die die Untersuchung durchführt.

Mit Ihrer Teilnahme an der Untersuchung helfen Sie uns weiter, chronische Schmerzsyndrome genauer zu verstehen und die Therapiemaßnahmen für Menschen mit chronischen Schmerzen zu verbessern. 


\section{Information für Teilnehmer}

Liebe Teilnehmerin, lieber Teilnehmer,

im wesentlichen geht es bei dieser Untersuchung darum, wie Sie auf verschiedene Bilder, die wir Ihnen über Monitor zeigen, reagieren. Und zwar interessiert uns besonders Ihre Augenmuskelreaktion.

Wir wollen untersuchen, ob die Muskelreaktion etwas darüber aussagt, ob Sie die Bilder langweilig oder interessant finden.

Dafür werden unter Ihrem Auge ganz kleine Meßfühler befestigt, die die Aktivität des Muskels, der für den Lidschlag zuständig ist, erfassen können. Sie werden davon fast gar nichts spüren.

Zu gewissen Zeiten während des Untersuchungsdurchlaufs werden Sie über Kopfhörer ein sehr kurzes lautes Geräusch hören. Lassen Sie sich dadurch in der Betrachtung der Bilder nicht stören.

Vor dem Beginn der Untersuchung möchten wir Sie noch bitten, einen Fragebogen auszufüllen. Danach werden wir Ihnen noch einige Fragen zu den Bildern stellen.

Der genaue Ablauf der Untersuchung und was Sie zu tun haben werden wir Ihnen noch im einzelnen erklären.

Sie können den Versuch jederzeit ohne Angabe von Gründen abbrechen. Das Abbrechen des Versuchs ist für Sie mit keinerlei negativen Folgen verbunden.

Wenn sie noch Fragen zur allgemeinen Durchführung haben, stellen Sie sie

bitte jetzt. Natürlich können Sie auch noch zu einem späteren Zeitpunkt

Fragen stellen, wenn Ihnen etwas unklar sein sollte.

Erklären Sie bitte Ihr Einverständnis mit dem Versuch und dokumentieren sie dies mit Ihrer Unterschrift.

\section{Einverständniserklärung:}

Ich bin über den Versuch schriftlich und mündlich aufgeklärt

worden. Ich erkläre mich damit einverstanden, daran teilzunehmen.

Ich weiß, daß ich den Versuch jederzeit abbrechen kann. Mit dem Abbrechen des Versuchs sind für mich keine Nachteile verbunden. 
Angaben zur Person: (die kursiv gedruckten Zeilen werden von der Versuchsleiterin ausgefüllt)

Name:

Code:

Geburtsdatum:

Gruppe:

Datum:

höchste

abgeschlossene

Schulausbildung:

Beruf:

Bemerkungen:

Hiermit erkläre ich mich mit der computergestützten Aufbewahrung und Auswertung der Daten einverstanden. Alle Daten werden streng vertraulich behandelt. Bei der Auswertung werden die Daten anonymisiert verwendet.

Datum, Unterschrift 
Codenr.:

Seit wann leiden Sie unter Ihren Kopfschmerzen?

Wie oft leiden Sie unter Ihren Kopfschmerzen?

Wenn Ihnen die medizinische Diagnose für Ihre Kopfschmerzen bekannt ist, bitte geben Sie diese an:

Sind Sie deswegen in Behandlung? ja $\quad \mathrm{O}$ nein $\mathrm{O}$

Wenn ja, bei welcher Institution?

Hausarzt

$\mathrm{O}$

Neurologe

$\mathrm{O}$

Schmerzambulanz

$\mathrm{O}$

andere

$\mathrm{O}$

Wenn andere, welche?

Seit wann sind Sie wegen der Kopfschmerzen in Behandlung?

Sind Sie zur Zeit aufgrund Ihrer Schmerzen arbeitsunfähig?

ja $\quad \mathrm{O}$

nein $\mathrm{O}$

Nehmen Sie zur Zeit regelmäßig Medikamente? Bitte geben Sie an welche und welche Medikamente Sie gegebenenfalls heute eingenommen haben. 
Instruktion 1. Teil

Erläuterungen zum Untersuchungsablauf:

Als erstes werde ich Ihnen diese beiden Meßfühler unter dem linken Auge anbringen und einen weiteren etwas größeren hinter dem Ohr.

Dafür muß die Haut ein wenig mit Alkohol gereinigt werden. Auf die Meßfühler wird etwas Gel aufgetragen.

Wenn Sie während der Untersuchung den Eindruck haben, daß sich ein Meßfühler gelöst hat, sagen Sie bitte gleich Bescheid.

Die Meßfühler werden nachher von mir wieder entfernt. Machen Sie dies bitte nicht selber. Die Fühler sind sehr empfindlich. 
Instruktion 2. Teil

Erläuterungen zum Untersuchungsablauf:

Setzen Sie nun bitte den Kopfhörer auf.

Setzen Sie sich mit dem Rücken an die Stuhllehne mit dem Blick auf den Monitor.

Rücken Sie mit dem Stuhl bis zur Markierung an den Tisch heran.

Sie werden gleich verschiedene Bilder zu sehen bekommen.

Schauen Sie sich diese Bilder aufmerksam an.

Auf den Bildern sind Menschen dargestellt, die verschiedene Bewegungen machen. Bitte versuchen Sie sich bei der Betrachtung der Bilder vorzustellen, daß Sie die abgebildete Bewegung durchführen.

Bitte schauen Sie die ganze Zeit direkt auf den Monitor, auch wenn darauf kein Bild erscheint. Zwischen den Bildern sehen Sie auf dem Monitor ein Kreuz. Schauen Sie bitte immer auf dieses Kreuz.

Es ist sehr wichtig, daß Sie während des Versuchs keine starken Bewegungen machen und vor allem den Kopf ruhighalten.

Während des Bilderanschauens werden Sie von Zeit zu Zeit ein kurzes lautes Geräusch über den Kopfhörer hören, auf das Sie aber nicht weiter zu achten brauchen. Gucken Sie einfach aufmerksam weiter die Bilder bzw. das Kreuz an.

Ich werde während Sie die Bilder anschauen, auf der anderen Seite der Wand sitzen. Wenn die Bilderserie beendet ist (nach ungefähr 15 Minuten), werde ich wieder hereinkommen. Dann wird der Kopfhörer abgesetzt und ich werde Ihnen den weiteren Verlauf der Untersuchung erläutern.

Jetzt gehe ich zunächst einmal kurz auf die andere Seite der Wand, um zu überprüfen, ob die Meßfühler richtig sitzen. Ich komme dann noch einmal kurz herein bevor die Bilder gezeigt werden. 
Instruktion 3. Teil

Erläuterungen zum Untersuchungsablauf:

Nun möchten wir Sie bitten, die Bilder noch einmal anzuschauen und für jedes einzelne Bild auf dem vor Ihnen liegenden Bogen anzugeben, welche Gefühle dieses Bild in Ihnen ausgelöst hat und wie stark dies Gefühl war. Außerdem möchten wir noch wissen, ob Sie bei diesem Bild das Geräusch über den Kopfhörer gehört haben oder nicht.

Wenn Sie mit dem Bogen für ein Bild fertig sind, können Sie selbst mit Hilfe der Maus das nächste Bild aufrufen.

Wenn Sie mit allen Bildern fertig sind, sagen Sie bitte Bescheid.

Bitte achten Sie stets darauf, daß das Bild auf dem Monitor mit dem kleinen Bild auf dem Bogen übereinstimmt, so daß wir genau wissen, welches Bild Sie gerade eingeschätzt haben.

Wenn Sie noch weitere Fragen haben, können Sie sie jetzt stellen. 
Instruktion 4. Teil

Erläuterungen zum Untersuchungsablauf:

Setzen Sie nun bitte nochmals den Kopfhörer auf.

Setzen Sie sich wieder mit dem Rücken an die Stuhllehne mit dem Blick auf den Monitor. Sie werden gleich weitere Bilder zu sehen bekommen.

Schauen Sie sich diese Bilder wieder aufmerksam an. Diesmal sollen Sie sich die Bilder anschauen, ohne sich in die dort dargestellten Situationen hineinzuversetzen.

Bitte schauen Sie wieder die ganze Zeit direkt auf den Monitor, auch wenn darauf kein Bild erscheint. Zwischen den Bildern sehen Sie auf dem Monitor ein Kreuz. Schauen Sie bitte immer auf dieses Kreuz.

Auch jetzt ist es wieder sehr wichtig, daß Sie während des Versuchs keine starken Bewegungen machen und vor allem den Kopf ruhighalten.

Wie vorhin werden Sie während des Bilderanschauens von Zeit zu Zeit ein kurzes lautes Geräusch über den Kopfhörer hören, auf das Sie aber nicht weiter zu achten brauchen. Gucken Sie einfach aufmerksam weiter die Bilder bzw. das Kreuz an.

Wenn die Bilderserie beendet ist (nach ungefähr 15 Minuten), werde ich wieder hereinkommen. Dann wird der Kopfhörer abgesetzt und ich werde die Meßfühler entfernen. Sie können dann einen Moment aufstehen und sich strecken. 
Instruktion 5. Teil

Erläuterungen zum Untersuchungsablauf:

Nun möchten wir Sie wieder bitten, die Bilder noch einmal anzuschauen und für jedes einzelne Bild auf dem vor Ihnen liegenden Bogen anzugeben, welche Gefühle dieses Bild in Ihnen ausgelöst hat und wie stark dies Gefühl war. Außerdem möchten wir noch wissen, ob Sie bei diesem Bild das Geräusch über den Kopfhörer gehört haben oder nicht.

Wenn Sie mit dem Bogen für ein Bild fertig sind können Sie selbst mit Hilfe der Maus das nächste Bild aufrufen.

Wenn Sie mit allen Bildern fertig sind, sagen Sie bitte Bescheid.

Bitte achten Sie stets darauf, daß das Bild auf dem Monitor mit dem kleinen Bild auf dem Bogen übereinstimmt, so daß wir genau wissen, welches Bild Sie gerade eingeschätzt haben. 


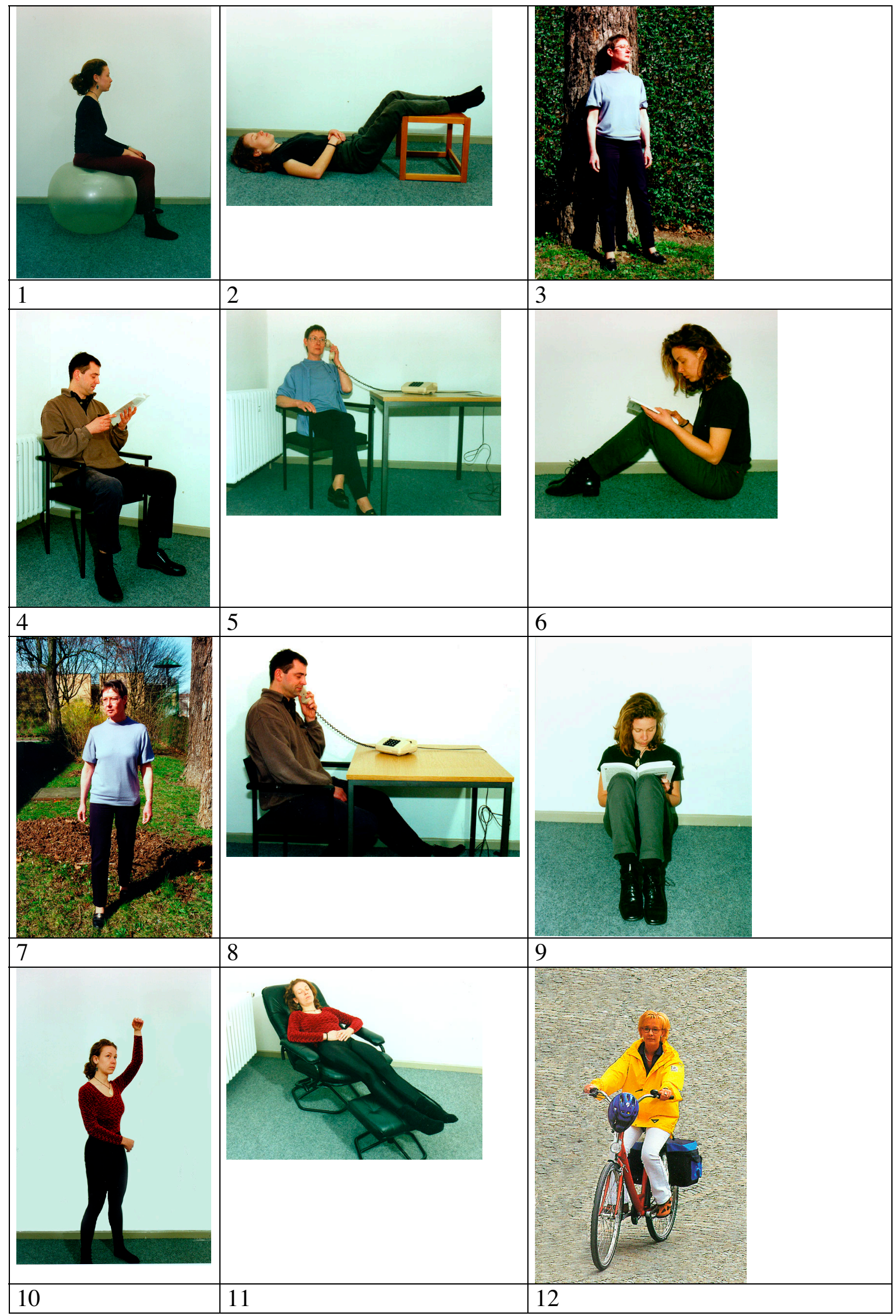




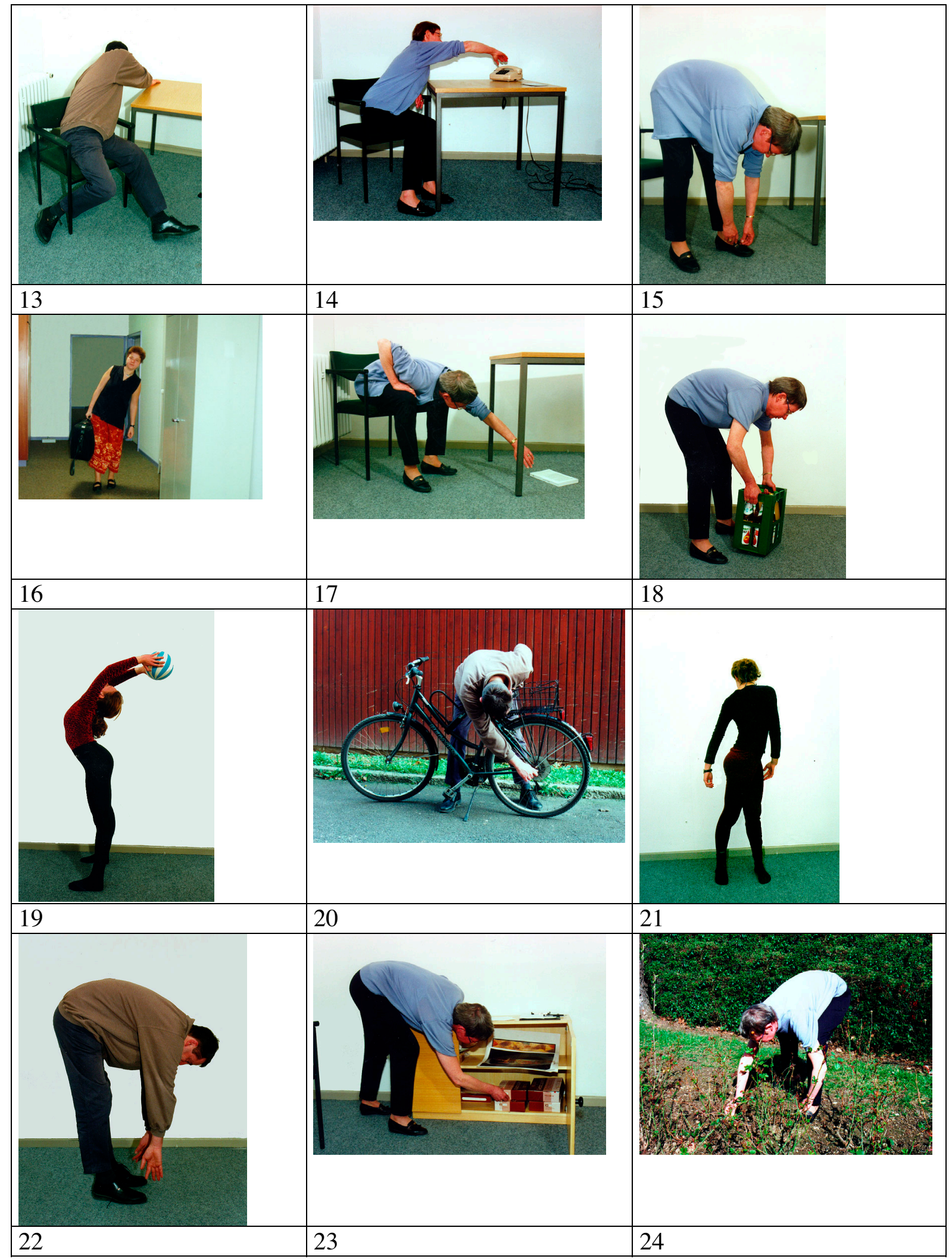




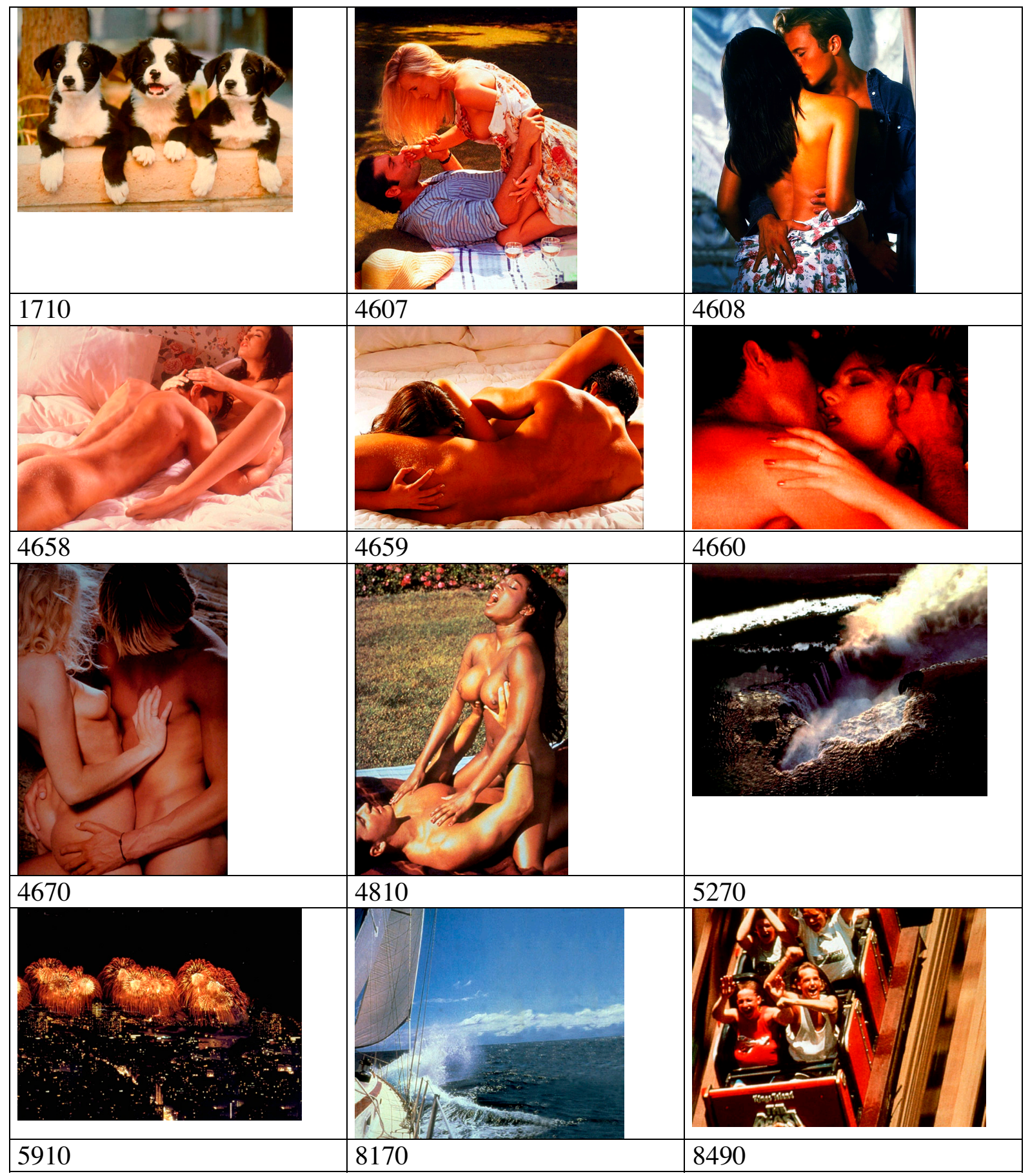




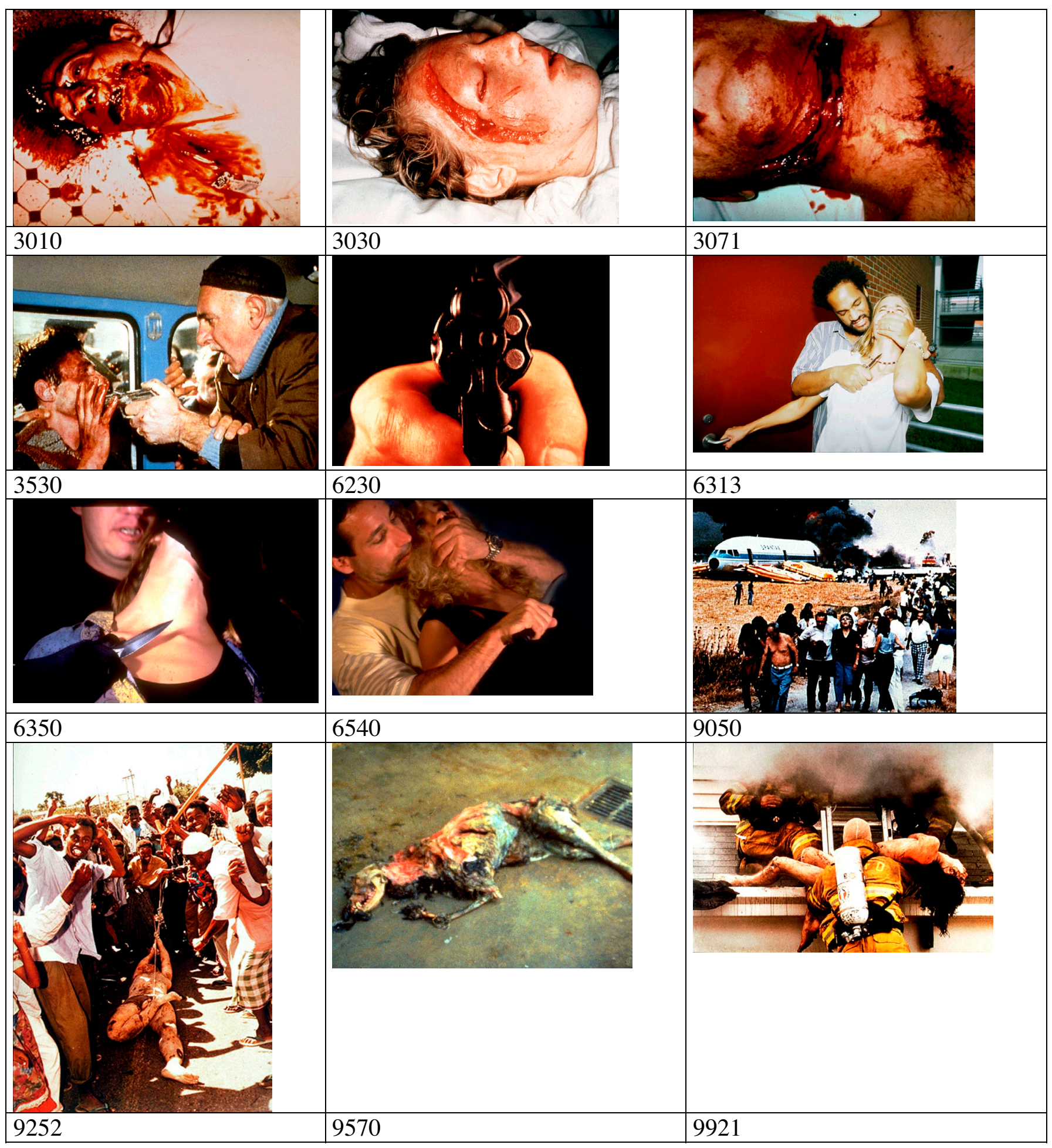


Tabelle B5.1 Verteilung der Bilder auf die Reihenfolgen: Bewegungsbilder

\begin{tabular}{|c|c|c|c|c|c|}
\hline $\begin{array}{c}\text { Reihenfolge } \\
1\end{array}$ & $\begin{array}{c}\text { Reihenfolge } \\
2\end{array}$ & $\begin{array}{c}\text { Reihenfolge } \\
3\end{array}$ & $\begin{array}{c}\text { Reihenfolge } \\
4\end{array}$ & $\begin{array}{c}\text { Reihenfolge } \\
5\end{array}$ & $\begin{array}{c}\text { Reihenfolge } \\
6\end{array}$ \\
\hline $1+$ & $11+$ & $5+$ & $6+$ & $2+$ & $18-$ \\
\hline 14- & 17- & 13- & 23- & 17- & $5+$ \\
\hline $21-$ & $22-$ & 14- & $12+$ & 21- & $9+$ \\
\hline $2+$ & $4+$ & $10+$ & 16- & $9+$ & 24- \\
\hline $3+$ & $7+$ & $9+$ & $8+$ & $5+$ & $4+$ \\
\hline 17- & 21- & 15- & 19- & 16- & 20- \\
\hline $4+$ & $1+$ & $2+$ & $7+$ & $8+$ & $11+$ \\
\hline 20- & 14- & 16- & 13- & 23- & 19- \\
\hline 18- & $20-$ & 18- & 22- & 14- & 21- \\
\hline $5+$ & $10+$ & $8+$ & $9+$ & $6+$ & $2+$ \\
\hline 15- & 23- & 17- & 24- & $22-$ & 15- \\
\hline $6+$ & $12+$ & $11+$ & $3+$ & $3+$ & $10+$ \\
\hline 23- & 13- & 19- & 17- & 19- & 14- \\
\hline $7+$ & $5+$ & $1+$ & $2+$ & $11+$ & $7+$ \\
\hline 19- & 24- & 20- & 15- & 15- & 23- \\
\hline $8+$ & $3+$ & $12+$ & $11+$ & $7+$ & $3+$ \\
\hline $9+$ & $6+$ & $4+$ & $18-$ & $4+$ & $1+$ \\
\hline 16- & 16- & 21- & $10+$ & 13- & 22- \\
\hline $10+$ & $9+$ & $3+$ & $5+$ & $12+$ & $8+$ \\
\hline 24- & 15- & 22- & 21- & 20- & 17- \\
\hline $11+$ & $8+$ & $7+$ & $4+$ & $10+$ & $12+$ \\
\hline 22- & 19- & 23- & $20-$ & 24- & 16- \\
\hline 13- & 18- & 24- & 14- & 18- & 13- \\
\hline $12+$ & $2+$ & $6+$ & $1+$ & $1+$ & $6+$ \\
\hline
\end{tabular}

14- Bild mit Startle-Reiz präsentiert 
Tabelle B6.1 Verteilung der Bilder auf die Reihenfolgen: IAPS-Bilder

\begin{tabular}{|c|c|c|c|c|c|}
\hline $\begin{array}{c}\text { Reihenfolge } \\
1 \\
\end{array}$ & $\begin{array}{c}\text { Reihenfolge } \\
2 \\
\end{array}$ & $\begin{array}{c}\text { Reihenfolge } \\
3 \\
\end{array}$ & $\begin{array}{c}\text { Reihenfolge } \\
4 \\
\end{array}$ & $\begin{array}{c}\text { Reihenfolge } \\
5 \\
\end{array}$ & $\begin{array}{c}\text { Reihenfolge } \\
6 \\
\end{array}$ \\
\hline $5910+$ & $8170+$ & $4659+$ & $4608+$ & $6540-$ & $4608+$ \\
\hline 9570- & 3010- & 9570- & 3071- & $4658+$ & 9921- \\
\hline 6313- & 3030- & 9252- & 9921- & $6350-$ & $9252-$ \\
\hline $4810+$ & $4660+$ & $5270+$ & $5270+$ & $8170+$ & $8490+$ \\
\hline 9252- & $6230-$ & $3530-$ & 9050- & 3530- & 9570- \\
\hline $1710+$ & $4608+$ & $4607+$ & $4660+$ & $4670+$ & $4660+$ \\
\hline 9921- & $1710+$ & $6540-$ & 3530- & $5270+$ & $8170+$ \\
\hline $4607+$ & $6350-$ & $4658+$ & $4659+$ & 3071- & $6230-$ \\
\hline $4660+$ & $5910+$ & $8490+$ & $8170+$ & $4608+$ & $1710+$ \\
\hline $6230-$ & 9252- & 9921- & 9570- & 6313- & $3010-$ \\
\hline $4670+$ & 9050- & $4810+$ & $8490+$ & $5910+$ & $4659+$ \\
\hline $6540-$ & $4670+$ & 6313- & $6230-$ & 3030- & 9050- \\
\hline $3071-$ & 9921- & $6350-$ & $6350-$ & 9570- & $6540-$ \\
\hline $8170+$ & $4659+$ & $4608+$ & $4607+$ & $4607+$ & $4658+$ \\
\hline $4659+$ & $4607+$ & $4670+$ & $4670+$ & $1710+$ & $4810+$ \\
\hline $3030-$ & 6540- & 9050- & $3010-$ & 9050- & 6350- \\
\hline $8490+$ & $5270+$ & $5910+$ & $4658+$ & $4660+$ & $5270+$ \\
\hline 3530- & 6313- & 3071- & 9252- & 3010- & 3530- \\
\hline $4608+$ & $4810+$ & $4660+$ & $1710+$ & $8490+$ & 3030- \\
\hline 9050- & 9570- & $6230-$ & 6313- & $9252-$ & $4670+$ \\
\hline $6350-$ & 3530- & 3030- & $6540-$ & $6230-$ & 6313- \\
\hline $5270+$ & $8490+$ & $1710+$ & $4810+$ & $4659+$ & $4607+$ \\
\hline 3010- & 3071- & 3010- & $3030-$ & 9921- & 3071- \\
\hline $4658+$ & $4658+$ & $8170+$ & $5910+$ & $4810+$ & $5910+$ \\
\hline
\end{tabular}

9570- Bild mit Startle-Reiz präsentiert 


\section{Instruktion für die Bilderevaluation}

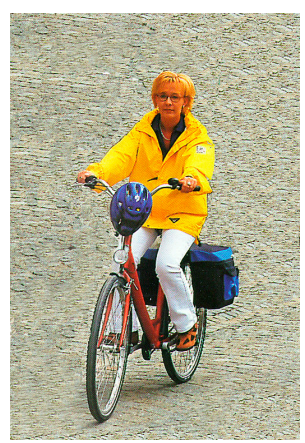

Schauen Sie sich jetzt bitte die Bilder nochmals einzeln an und beantworten Sie für jedes Bild die unten stehenden Fragen. Bitte achten Sie darauf, daß das Bild auf dem Monitor mit dem hier abgebildeten übereinstimmt.

Lassen Sie das Bild auf sich wirken und geben Sie an, welche Gefühle das Bild bei Ihnen auslöst.

Das vorliegende Bild empfinde ich als

$\begin{array}{ll}\text { (1)---(2)---(3)---(4)---(5)---(6)---(7)---(8)---(9) } \\ \text { sehr } & \text { sehr } \\ \text { negativ } & \text { positiv }\end{array}$

Bitte geben Sie an, wie stark Sie dies Gefühl empfinden:

Ich empfinde das oben genannte Gefühl

$\begin{array}{lr}\text { (1)---(2)---(3)---(4)---(5)---(6)---(7)---(8)---(9) } \\ \text { sehr } & \text { sehr } \\ \text { schwach } & \text { stark }\end{array}$

Bitte geben Sie nun noch an, ob Sie bei diesem Bild das Geräusch über Kopfhörer gehört haben:

$$
\begin{array}{ll}
\bigcirc & \text { ja } \\
\bigcirc & \text { nein }
\end{array}
$$


Fear-Avoidance-Beliefs Questionnaire, deutsche Version

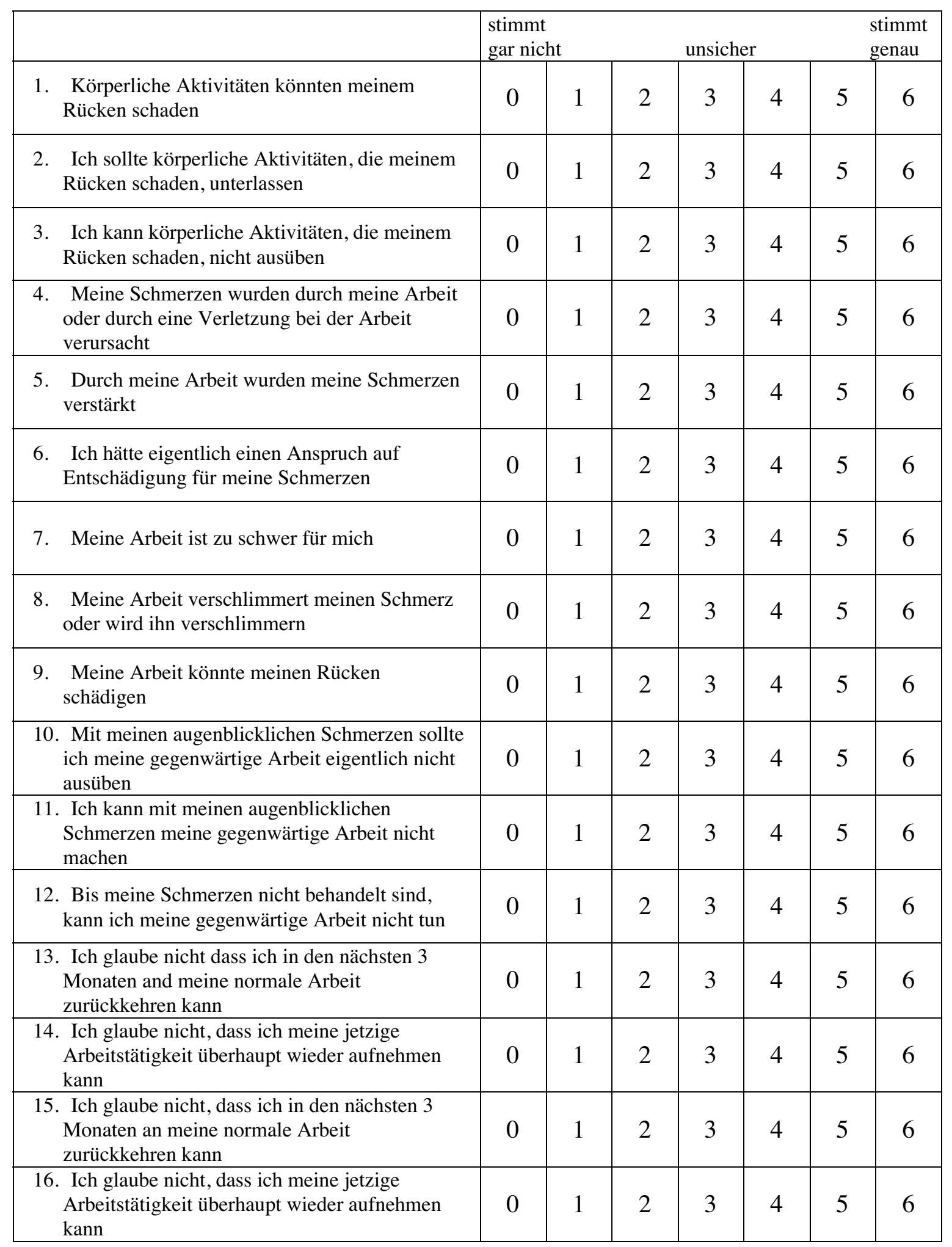




\section{SAB}

Wenn ich Schmerzen habe...

$\begin{array}{ccccc}\text { trifft } & \text { trifft } & \text { trifft } & \text { trifft } & \text { trifft } \\ \text { genau } & \mathrm{zu} & \text { eher } & \text { nicht } & \text { überhaupt } \\ \text { zu } & & \text { zu } & \text { zu } & \text { nicht } \\ & & & & \text { zu }\end{array}$

1. fühle ich mich beunruhig

$\mathrm{O}$

$\mathrm{O}$

$\mathrm{O}$

$\mathrm{O}$

$\mathrm{O}$

2. fühle ich mich gestresst und belastet

$\mathrm{O}$

$\mathrm{O}$

$\mathrm{O}$

$\mathrm{O}$

$\mathrm{O}$

3. fürchte ich mich vor allem, was auf mich zukommen könnte.

$\begin{array}{lllll}\mathrm{O} & \mathrm{O} & \mathrm{O} & \mathrm{O} & \mathrm{O}\end{array}$

4. habe ich Angst, den

Anforderungen des Alltags nicht

$\mathrm{O}$

$\mathrm{O}$

$\mathrm{O}$

$\mathrm{O}$

$\mathrm{O}$ mehr gerecht werden zu können.

5. bin ich nervös und angespannt

$\mathrm{O}$

$\mathrm{O}$

$\mathrm{O}$

$\mathrm{O}$

$\mathrm{O}$

6. habe ich Angst, die Kontrolle zu verlieren

$\mathrm{O}$

$\mathrm{O}$

$\mathrm{O}$

$\mathrm{O}$

$\mathrm{O}$

7. bekomme ich Atemnot

$\mathrm{O}$

$\mathrm{O}$

$\mathrm{O}$

$\mathrm{O}$

$\mathrm{O}$

8. fühle ich mich zittrig

$\mathrm{O}$

O

$\mathrm{O}$

$\mathrm{O}$

$\mathrm{O}$

9. befürchte ich das Schlimmste

$\mathrm{O}$

$\mathrm{O}$

$\mathrm{O}$

$\mathrm{O}$

$\mathrm{O}$

10. kann ich mich auf nichts anderes mehr konzentrieren

$\mathrm{O}$
$\mathrm{O}$

$\mathrm{O}$

$\mathrm{O}$

$\mathrm{O}$

11. fange ich an zu schwitzen

$\mathrm{O}$

O

$\mathrm{O}$

$\mathrm{O}$

$\mathrm{O}$

12. bekomme ich Herzklopfen

$\mathrm{O}$

$\mathrm{O}$

$\mathrm{O}$

$\mathrm{O}$

$\mathrm{O}$

13. grüble ich voller Sorge die ganze

Zeit über die Schmerzen nach

$\mathrm{O}$

$\mathrm{O}$

$\mathrm{O}$

$\mathrm{O}$

$\mathrm{O}$

14. habe ich Angst, dass ich den

Schmerzen nicht gewachsen sein

$\mathrm{O}$

O

$\mathrm{O}$

$\mathrm{O}$

$\mathrm{O}$ könnte

15. gehen mir alle möglichen Sorgen durch den Kopf

$\mathrm{O}$

$\mathrm{O}$

$\mathrm{O}$

$\mathrm{O}$

O 
SEB

1. Wer Schmerzen hat, sollte sich schonen

$\begin{array}{ccccc}\text { trifft } & \text { trifft } & \text { trifft } & \text { trifft } & \text { trifft } \\ \text { genau } & \text { zu } & \text { eher } & \text { nicht } & \text { überhaupt } \\ \text { zu } & & \text { zu } & \text { zu } & \text { nicht } \\ & & & & \text { zu }\end{array}$

$\begin{array}{lllll}\mathrm{O} & \mathrm{O} & \mathrm{O} & \mathrm{O} & \mathrm{O}\end{array}$

2. Ein Mensch, der Schmerzen hat, sollte sich möglichst wenig bewegen

$\begin{array}{lllll}\mathrm{O} & \mathrm{O} & \mathrm{O} & \mathrm{O} & \mathrm{O}\end{array}$

3. Medikamente und Spritzen sind die wirksamsten Mittel gegen Schmerzen

$\begin{array}{lllll}\mathrm{O} & \mathrm{O} & \mathrm{O} & \mathrm{O} & \mathrm{O}\end{array}$

4. Menschen, die an Schmerzen leiden, sollten eher nicht zur Arbeit gehen

$\begin{array}{lllll}\mathrm{O} & \mathrm{O} & \mathrm{O} & \mathrm{O} & \mathrm{O}\end{array}$

5. Alles, was Schmerzen verschlimmert, sollte möglichst vermieden werden

$\begin{array}{lllll}\mathrm{O} & \mathrm{O} & \mathrm{O} & \mathrm{O} & \mathrm{O}\end{array}$

6. Wer Schmerzen hat, kann normalerweise keinen Sport treiben

$\begin{array}{lllll}\mathrm{O} & \mathrm{O} & \mathrm{O} & \mathrm{O} & \mathrm{O}\end{array}$

7. Wer Schmerzen hat, isst zu vielen alltäglichen Verrichtungen nicht in der Lage

8. Wer Schmerzen hat, kann normalerweise keine Freunde und Bekannte treffen oder andere soziale Kontakte pflegen.

9. Die meisten Menschen, die an Schmerzen leiden, sind nicht arbeitsfähig

10. Wer Schmerzen hat, kann normalerweise seinen Hobbys nicht mehr nachgehen

$\begin{array}{lllll}\mathrm{O} & \mathrm{O} & \mathrm{O} & \mathrm{O} & \mathrm{O}\end{array}$

$\begin{array}{lllll}\mathrm{O} & \mathrm{O} & \mathrm{O} & \mathrm{O} & \mathrm{O}\end{array}$

$\begin{array}{lllll}\mathrm{O} & \mathrm{O} & \mathrm{O} & \mathrm{O} & \mathrm{O}\end{array}$

$\begin{array}{lllll}\mathrm{O} & \mathrm{O} & \mathrm{O} & \mathrm{O} & \mathrm{O}\end{array}$

11. Wer Schmerzen hat, braucht vor allen Dingen Ruhe

$\begin{array}{lllll}\mathrm{O} & \mathrm{O} & \mathrm{O} & \mathrm{O} & \mathrm{O}\end{array}$ 
ABB

Bitte nehmen Sie auf den nachfolgenden Seiten Stellung zu Ihrer Zufriedenheit mit Ihrer gegenwärtigen Arbeit. Bitte gehen Sie die einzelnen Bereiche durch und kreuzen Sie an, wie zufrieden Sie jeweils in diesem Bereich sind.

1. Kollegen: Gemeint sind die Kolleginnen und Kollegen, mit denen Sie unmittelbar zusammenarbeiten und arbeitsbezogenen Kontakt haben.

Alles in allem: Wie zufrieden sind Sie mit Ihren Kollegen?

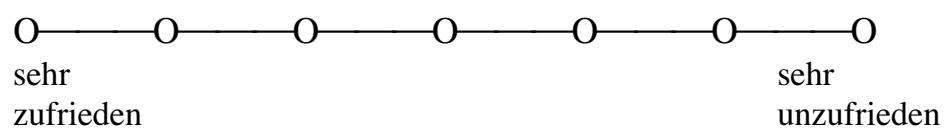

2. Vorgesetzter : Gemeint ist Ihr unmittelbarer Vorgesetzter, der Ihnen Anweisungen geben und Sie kontrollieren kann.

Alles in allem: Wie zufrieden sind Sie mit Ihrem Vorgesetzten?

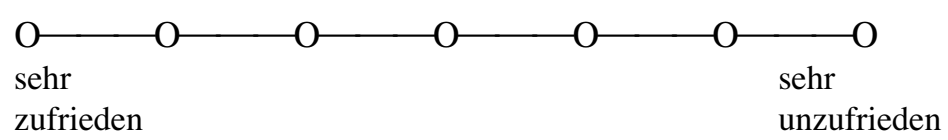

3. Tätigkeit: Gemeint ist der Inhalt Ihrer Tätigkeit, die Art Ihrer Arbeitsaufgaben.

Alles in allem: Wie zufrieden sind Sie mit Ihrer Tätigkeit?

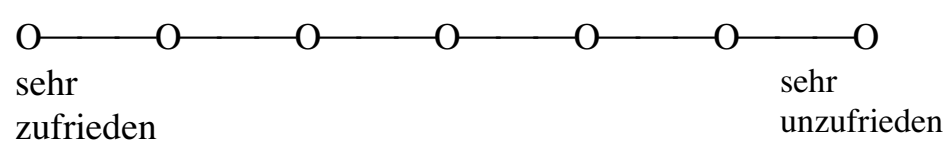

4. Arbeitsbedingungen: Gemeint sind die Bedingungen, unter denen Sie arbeiten (z.B.Hilfsmittel, Arbeitsraum, Umgebung, Lärm etc.).

Alles in allem: Wie zufrieden sind Sie mit den Arbeitsbedingungen?

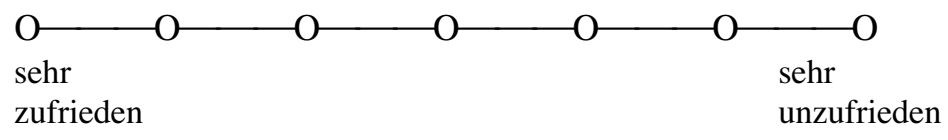

5. Organisation und Leitung: Gemeint ist, wie Sie die Firma als ganzes sehen, wie die Zusammenarbeit zwischen den Bereichen und Abteilungen funktioniert, wie Sie Vorschriften und Regeln, Planung und Information und die Leitung der Firma beurteilen.

Alles in allem : Wie zufrieden sind Sie mit der Organisation und Leitung?

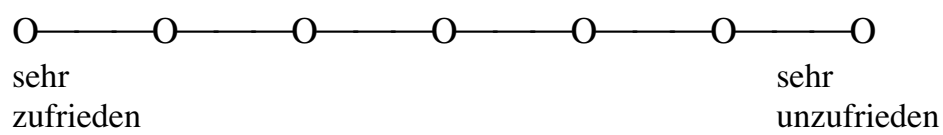


6. Eigene Entwicklung: Gemeint ist Ihr persönliches Vorwärtskommen

(Ihre bisherigen und zukünftigen Möglichkeiten zum Aufstieg, zur

Weiterbildung und zur Übernahme von verantwortungsvolleren

Aufgaben).

Alles in allem: Wie zufrieden sind Sie mit Ihren

Entwicklungsmöglichkeiten?

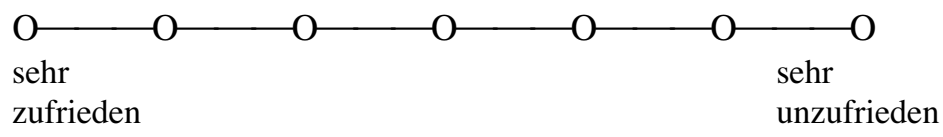

7. Bezahlung: Gemeint ist die Höhe Ihrer Bezahlung, die Sie für Ihre Arbeit erhalten (einschließlich Zulagen, Zuschläge, 13. Monatsgehalt etc.).

Alles in allem: Wie zufrieden sind Sie mit Ihrer Bezahlung?

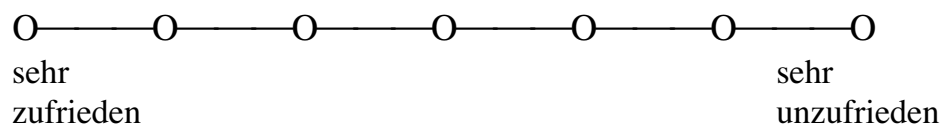

8. Arbeitszeit: Gemeint ist die Einteilung der Arbeitszeit.

Alles in allem: Wie zufrieden sind Sie mit der Einteilung Ihrer Arbeitszeit?

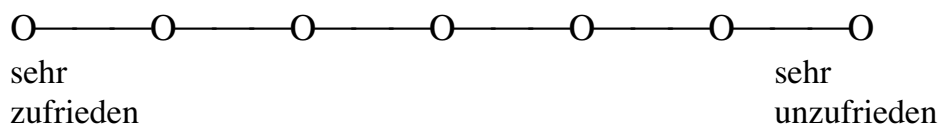

9. Sicherheit des Arbeitsplatzes: Wie hoch schätzen Sie die Sicherheit ein, Ihren Arbeitsplatz zu behalten?

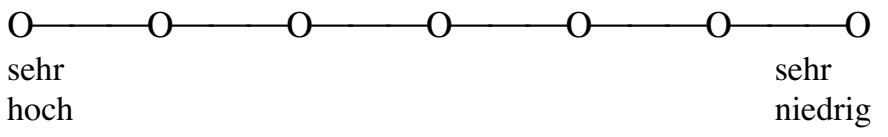

10. Gesamt: Wenn Sie nun an alles denken, was für Ihre Arbeit eine Rolle spielt, wie zufrieden sind Sie dann insgesamt mit Ihrer Arbeit?

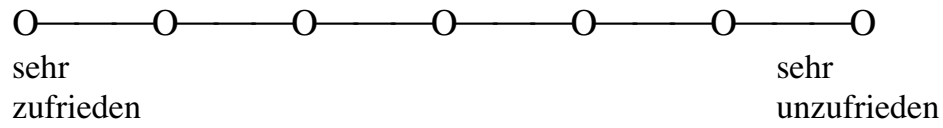




\begin{tabular}{|c|c|c|c|c|}
\hline $\begin{array}{l}\text { Anleitung: Im folgenden Fragebogen finden Sie eine } \\
\text { Reihe von Feststellungen, mit denen man sich selbst } \\
\text { beschreiben kann. Bitte lesen Sie jede Feststellung durch } \\
\text { und wählen Sie aus den vier Antworten diejenige aus, die } \\
\text { angibt, wie Sie sich jetzt, d.h. in diesem Moment, } \\
\text { fühlen. Kreuzen Sie bitte bei jeder Feststellung die Zahl } \\
\text { unter der von Ihnen gewählten Antwort an. } \\
\text { Es gibt keine richtigen oder falschen Antworten. Überlegen Sie bitte nicht } \\
\text { lange und denken Sie daran, diejenige Antwort auszuwählen, die Ihren } \\
\text { augenblicklichen Gefühlszustand am besten beschreibet. }\end{array}$ & 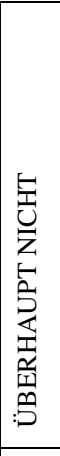 & 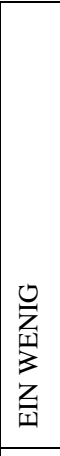 & 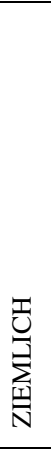 & 岛 \\
\hline 1. Ich bin ruhig & 1 & 2 & 3 & 4 \\
\hline 2. Ich fühle mich geborgen & 1 & 2 & 3 & 4 \\
\hline 3. Ich fühle mich angespannt & 1 & 2 & 3 & 4 \\
\hline 4. Ich bin bekümmert & 1 & 2 & 3 & 4 \\
\hline 5. Ich bin gelöst & 1 & 2 & 3 & 4 \\
\hline 6. Ich bin aufgeregt & 1 & 2 & 3 & 4 \\
\hline 7. Ich bin besorgt, daß etwas schiefgehen könnte & 1 & 2 & 3 & 4 \\
\hline 8. Ich fühle mich ausgeruht & 1 & 2 & 3 & 4 \\
\hline 9. Ich bin beunruhigt & 1 & 2 & 3 & 4 \\
\hline 10. Ich fühle mich wohl & 1 & 2 & 3 & 4 \\
\hline 11. Ich fühle mich selbstsicher & 1 & 2 & 3 & 4 \\
\hline 12. Ich bin nervös & 1 & 2 & 3 & 4 \\
\hline 13. Ich bin zappelig & 1 & 2 & 3 & 4 \\
\hline 14. Ich bin verkrampft & 1 & 2 & 3 & 4 \\
\hline 15. Ich bin entspannt & 1 & 2 & 3 & 4 \\
\hline 16. Ich bin zufrieden & 1 & 2 & 3 & 4 \\
\hline 17. Ich bin besorgt & 1 & 2 & 3 & 4 \\
\hline 18. Ich bin überreizt & 1 & 2 & 3 & 4 \\
\hline 19. Ich bin froh & 1 & 2 & 3 & 4 \\
\hline 20. Ich bin vergnügt & 1 & 2 & 3 & 4 \\
\hline
\end{tabular}


STAI-Trait

\begin{tabular}{|c|c|c|c|c|}
\hline $\begin{array}{l}\text { Anleitung: Im folgenden Fragebogen finden Sie eine Reihe } \\
\text { von Feststellungen, mit denen man sich selbst beschreiben } \\
\text { kann. Bitte lesen Sie jede Feststellung durch und wählen Sie } \\
\text { aus den vier Antworten diejenige aus, die angibt, wie Sie sich } \\
\text { im allgemeinen fühlen. Kreuzen Sie bitte bei jeder } \\
\text { Feststellung die Zahl unter der von Ihnen gewählten Antwort } \\
\text { an. } \\
\text { Es gibt keine richtigen oder falschen Antworten. Überlegen Sie bitte nicht lange und } \\
\text { denken Sie daran, diejenige Antwort auszuwählen, die am besten beschreibet., wie } \\
\text { Sie sich im allgemeinen fühlen. }\end{array}$ & 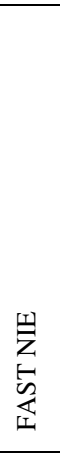 & 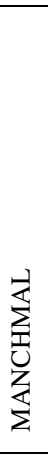 & 宁 & 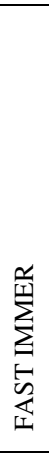 \\
\hline 21. Ich bin vergnügt & 1 & 2 & 3 & 4 \\
\hline 22. Ich werde schnell müde & 1 & 2 & 3 & 4 \\
\hline 23. Mir ist zum Weinen zumute & 1 & 2 & 3 & 4 \\
\hline 24. Ich glaube, mir geht es schlechter als anderen Leuten & 1 & 2 & 3 & 4 \\
\hline $\begin{array}{l}\text { 25. Ich verpasse günstige Gelegenheiten, weil ich mich nicht schnell } \\
\text { genug entscheiden kann }\end{array}$ & 1 & 2 & 3 & 4 \\
\hline 26. Ich fühle mich ausgeruht & 1 & 2 & 3 & 4 \\
\hline 27. Ich bin ruhig und gelassen & 1 & 2 & 3 & 4 \\
\hline 28. Ich glaube, dass mir meine Schwierigkeiten über den Kopf wachsen & 1 & 2 & 3 & 4 \\
\hline 29. Ich mache mir zuviel Gedanken über unwichtige Dinge & 1 & 2 & 3 & 4 \\
\hline 30. Ich bin glücklich & 1 & 2 & 3 & 4 \\
\hline 31. Ich neige dazu, alles schwer zu nehmen & 1 & 2 & 3 & 4 \\
\hline 32. Mir fehlt es an Selbstvertrauen & 1 & 2 & 3 & 4 \\
\hline 33. Ich fühle mich geborgen & 1 & 2 & 3 & 4 \\
\hline 34. Ich mache mir Sorgen über mögliches Mißgeschick & 1 & 2 & 3 & 4 \\
\hline 35. Ich fühle mich niedergeschlagen & 1 & 2 & 3 & 4 \\
\hline 36. Ich bin zufrieden & 1 & 2 & 3 & 4 \\
\hline $\begin{array}{l}\text { 37. Unwichtige Gedanken gehen mir durch den Kopf und bedrücken } \\
\text { mich }\end{array}$ & 1 & 2 & 3 & 4 \\
\hline $\begin{array}{l}\text { 38. Enttäuschungen nehme ich so schwer, dass ich sie nicht vergessen } \\
\text { kann }\end{array}$ & 1 & 2 & 3 & 4 \\
\hline 39. Ich bin ausgeglichen & 1 & 2 & 3 & 4 \\
\hline $\begin{array}{l}\text { 40. Ich werde nervös und unruhig, wenn ich an meine derzeitigen } \\
\text { Angelegenheiten denke }\end{array}$ & 1 & 2 & 3 & 4 \\
\hline
\end{tabular}


Vergleich der Experimentalgruppe mit einer Gruppe Patienten des Klinikums Göttingen in Bezug auf Beeinträchtigung und Schmerzstärke

Tabelle C1.1 Maße des FFbH und VAS Schmerzstärke, Experimentalgruppe und Gruppe aus Klinikum: Mittelwerte und Standardabweichungen ( )

\begin{tabular}{|l|l|l|}
\hline & FFbH & Schmerzstärke \\
\hline Experimentalgruppe $(\mathrm{n}=36)$ & $61.9(19.4)$ & $6,3(2.1)$ \\
\hline Gruppe Klinikum $(\mathrm{n}=190)$ & $63.2(21.4)$ & $6,3(1.9)$ \\
\hline
\end{tabular}

Tabelle C1.2 Unterschiede in FFbH und VAS Schmerzstärke zwischen den Gruppen: Ergebnisse der t-Tests

\begin{tabular}{|l|l|l|l|}
\hline & t-Wert & df & p \\
\hline FFbH & 0.33 & $1 / 218$ & 0.74 \\
\hline Schmerzstärke & 0.035 & $1 / 224$ & 0.98 \\
\hline
\end{tabular}


Berechnung der Startle-Reaktion aufgrund der Differenzwerte

Tabelle C2.1 Differenz der Startle-Reaktion (Bewegung : minus Bewegung (:)): Mittelwerte und Standardabweichungen ( ) (nur Responder)

\begin{tabular}{|l|l|l|}
\hline CLBP & KG KS & KG GES \\
\hline $21.0(142.8)$ & $-28.5(143.9)$ & $21.3(66.9)$ \\
\hline
\end{tabular}

Tabelle C2.2 Startle-Reaktion auf die Bewegungsbilder als Differenzwert: Ergebnisse der Varianzanalyse (nur Responder)

\begin{tabular}{|l|l|l|l|}
\hline & F & Df & p \\
\hline Gruppe (CLBP, KG KS, KG GES) & 0.9 & $2 / 56$ & 0.41 \\
\hline
\end{tabular}

Höhe der Startle-Reaktion auf die Startle-Reize in den Pausen (Blankstartle)

Tabelle C2.3 Mittelwert und Standardabweichung der Startle-Reaktion in den Pausen zwischen den Bildern (Blankstartle)

\begin{tabular}{|c|l|l|l|}
\hline & CLBP & KG KS & KG GES \\
\hline Blank / Bewegung & $360.6(294.2)$ & $367.6(323.1)$ & $346.1(253.2)$ \\
\hline Blank / IAPS & $343(193.4)$ & $368.9(292.7))$ & $308.7(193.4)$ \\
\hline
\end{tabular}




\section{Covariation Bias in Block 2}

Tabelle C3.1 Covariation Bias für Bewegungsbilder bei Block 2

\begin{tabular}{|l|l|l|l|}
\hline Gruppe & 1.14 & $1 / 33$ & 0.26 \\
\hline Qualität & 0.16 & $1 / 33$ & 0.69 \\
\hline Gruppe x Qualität & 3.41 & $1 / 33$ & $0.04 *$ \\
\hline
\end{tabular}

*p $\leq 0.05$

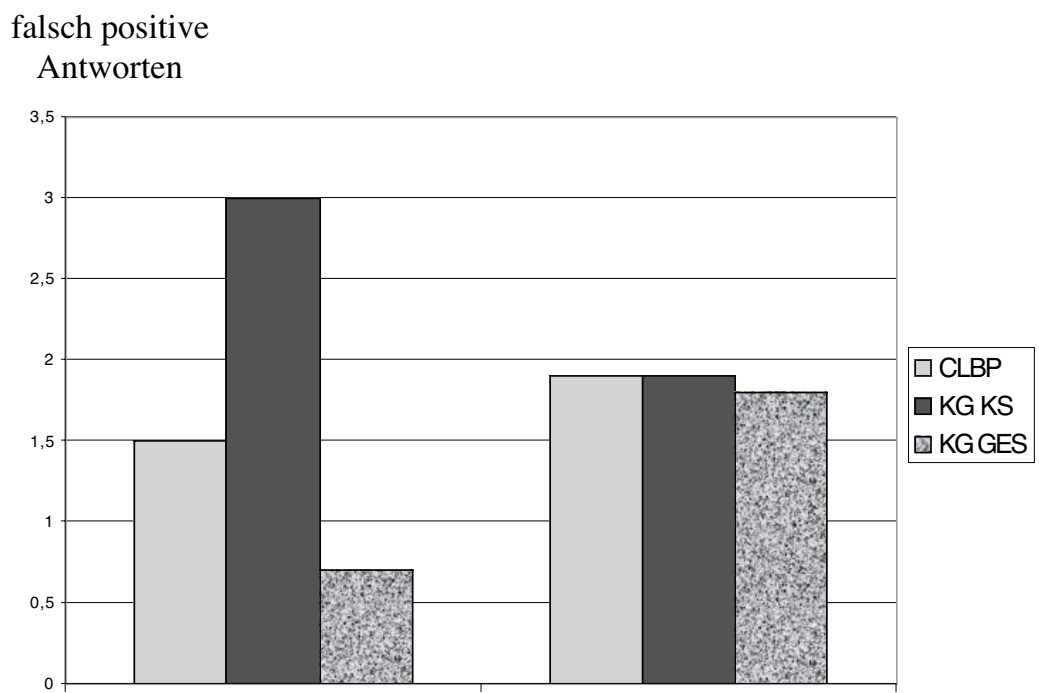

Bilder

$$
\text { Bewegung :) Bewegung }(;
$$

Abbildung C3.1 Covariation Bias bei den Bewegungbildern in Block 2 
Ergebnisse der Faktorenanalyse für den SAB

Tabelle C4.1 SAB: Eigenwerte und Varianzanteile der durch die Analyse vorgeschlagenen Faktoren

\begin{tabular}{l|r|r|}
\multicolumn{1}{c}{} & \multicolumn{1}{c}{ Betrag } & Varianzanteil \\
\cline { 2 - 3 } Wert 1 & 7,345 &, 490 \\
\cline { 2 - 3 } Wert 2 & 1,403 &, 094 \\
\cline { 2 - 3 } Wert 3 & 1,176 &, 078 \\
Wert 4 &, 888 &, 059 \\
Wert 5 &, 772 &, 051 \\
\cline { 2 - 3 } Wert 6 &, 688 &, 046 \\
\cline { 2 - 3 } Wert 7 &, 559 &, 037 \\
\cline { 2 - 3 }
\end{tabular}

Tabelle C4.2 SAB: Faktorladungen

\begin{tabular}{l|r|r|r|r|r|}
\multicolumn{1}{c}{} & Faktor 1 & Faktor 2 & \multicolumn{1}{r}{ Faktor 3 } & Faktor 4 & Faktor 5 \\
\cline { 2 - 6 } f1 &, 492 &, 048 &, 517 &, 547 &, 331 \\
\cline { 2 - 6 } f2 &, 684 &,- 473 &,- 258 &, 215 &, 166 \\
\cline { 2 - 6 } f3 &, 746 &,- 397 &,- 019 &,- 186 &,- 092 \\
\cline { 2 - 6 } f4 &, 796 &,- 135 &,- 113 &, 078 &,- 126 \\
\cline { 2 - 6 } f5 &, 703 &,- 351 &,- 388 &, 169 &,- 206 \\
\cline { 2 - 6 } f6 &, 780 &,- 178 &, 055 &, 067 &,- 074 \\
\cline { 2 - 6 } f7 &, 510 &, 569 &,- 275 &,- 218 &,- 074 \\
\cline { 2 - 6 } f &, 598 &, 452 &,- 369 &, 094 &, 231 \\
\cline { 2 - 6 } f9 &, 731 &, 285 &, 294 &,- 185 &, 090 \\
\cline { 2 - 6 } f10 &, 609 &,- 125 &,- 221 &,- 368 &, 534 \\
\cline { 2 - 6 } f11 &, 647 &, 370 &,- 032 &, 193 &,- 302 \\
\cline { 2 - 6 } f12 &, 749 &, 277 &,- 209 &, 263 &, 019 \\
\cline { 2 - 6 } f13 &, 789 &,- 015 &, 345 &,- 192 &, 156 \\
\cline { 2 - 6 } f14 &, 744 &,- 082 &, 229 &,- 313 &,- 209 \\
\cline { 2 - 6 } f15 &, 815 &, 028 &, 334 &,- 041 &,- 226 \\
\hline
\end{tabular}


Ergebnisse der Faktorenanalyse für den SEB

Tabelle C5.1 SEB: Eigenwerte und Varianzanteile der durch die Analyse vorgeschlagenen Faktoren

\begin{tabular}{l|r|r|}
\multicolumn{1}{c}{} & \multicolumn{1}{c}{ Betrag } & Varianzanteil \\
\cline { 2 - 3 } Wert 1 & 4,687 &, 426 \\
\cline { 2 - 3 } Wert 2 & 1,353 &, 123 \\
Wert 3 & 1,024 &, 093 \\
Wert 4 &, 759 &, 069 \\
\cline { 2 - 3 } Wert 5 &, 683 &, 062 \\
\cline { 2 - 3 }
\end{tabular}

Tabelle C5.2 SEB: Faktorladungen

\begin{tabular}{l|r|r|r|r|r|}
\multicolumn{1}{c}{} & \multicolumn{1}{c}{ Faktor 1 } & Faktor 2 & Faktor 3 & Faktor 4 & Faktor 5 \\
\cline { 2 - 6 } f1 &, 630 &,- 566 &,- 160 &, 108 &,- 210 \\
\cline { 2 - 6 } f2 &, 643 &,- 339 &,- 117 &,- 535 &, 073 \\
\cline { 2 - 6 } f3 &, 205 &,- 429 &, 818 &, 216 &,- 002 \\
\cline { 2 - 6 } f4 &, 740 &,- 131 &,- 103 &, 162 &,- 256 \\
\cline { 2 - 6 } f5 &, 659 &,- 167 &,- 322 &, 534 &, 192 \\
\cline { 2 - 6 } f6 &, 737 &,- 161 &,- 130 &,- 187 &, 205 \\
\cline { 2 - 6 } f7 &, 790 &, 196 &,- 052 &, 005 &, 018 \\
\cline { 2 - 6 } f8 &, 639 &, 240 &, 226 &, 006 &, 602 \\
\hline f9 &, 580 &, 647 &, 110 &, 136 &,- 186 \\
\cline { 2 - 6 } f10 &, 705 &, 381 &,- 004 &,- 066 &,- 206 \\
\cline { 2 - 6 } f11 &, 665 &, 055 &, 344 &,- 214 &,- 223 \\
\hline
\end{tabular}




\section{Interkorrelationen FABQ}

Tabelle C6.1 Interkorrelationen des FABQ mit seinen Subskalen

\begin{tabular}{|l|l|l|l|l|}
\hline & FABQ & Ursache Arbeit & Prognose Arbeit & Aktivität \\
\hline Ursache Arbeit & $.948 * *$ & & $.829 * *$ & $.567 * *$ \\
\hline Prognose Arbeit & $.901 * *$ & & & $.443 * *$ \\
\hline Aktivität & $.72 * *$ & & & \\
\hline
\end{tabular}




\section{Uta Kronshage}

Keplerstr. 3

37085 Göttingen

Tel. 0551 / 5311096

\section{Lebenslauf}

\section{Persönliche Daten}

Geburtsdatum

07.04.1965

Geburtsort

Göttingen

Familienstand

ledig

Staatsangehörigkeit

deutsch

\section{Schulbildung}

1971-1975

Grundschulbesuch in Hannover

1975-1984

Besuch des Gymnasiums Schillerschule Hannover

1984

Abitur

\section{Hochschulausbildung}

Oktober 1984

Aufnahme des Studiums der Fächer Deutsch und Französisch an der Universität Göttingen

Oktober 1985

Wechsel des Studienfaches

Oktober 1985 - Oktober 1992

Studium der Psychologie in Göttingen

30.10 .1992

Abschluss des Studiums mit Diplom

Schwerpunktfach: Klinische Psychologie

\section{Berufstätigkeit}

April 1993-Januar 1994

Dozentin für Psychologie an der

Hebammenschule der Universität Göttingen

Mai 1993-Februar 1994

Tätigkeit am Institut für Berufliche Bildung und Weiterbildung in Göttingen als Projektleiterin für die "Weiterbildung von Beratern und Betreuern von Aussiedlern und Flüchtlingen im Land Brandenburg" 


\section{Berufstätigkeit}

Mai 1994 - März 1996

Oktober 1996- September 1998

September 1998 - September 2001

\section{Berufliche Weiterbildungen}

Oktober 1993 bis Dezember 1996

Seit März 1997

Januar 1999

Göttingen, 17.09.2001
Tätigkeit als Dipl.-Psychologin, Klinik und Rehabilitationszentrum Lippoldsberg

Tätigkeit am Georg-Elias-Müller-Institut für Psychologie, Universität Göttingen, Ambulanz für Psychotherapie, Schwerpunkt: Behandlung von chronischen Schmerzerkrankungen

Angestellte am Georg-Elias-Müller-Institut für Psychologie in einem Projekt gefördert durch die DFG, Erstellung der Promotion

Verhaltenstherapeutische Weiterbildung zur "Klinischen Psychologin/Psychotherapeutin " in Zusammenarbeit der Technischen Universität Braunschweig mit der Nordwestdeutschen Psychologenakademie

Weiterbildung in „Klinischer Hypnose“ am Milton-Erikson-Institut in Göttingen

Approbation als Psychologische Psychotherapeutin, Fachkunde in Verhaltenstherapie 